TRANSACTIONS OF THE

AMERICAN MATHEMATICAL SOCIETY

Volume 361, Number 12, December 2009, Pages 6205-6253

S 0002-9947(09)04565-6

Article electronically published on July 14, 2009

\title{
SIGN-CHANGING MULTI-BUMP SOLUTIONS FOR NONLINEAR SCHRÖDINGER EQUATIONS WITH STEEP POTENTIAL WELLS
}

\author{
YOHEI SATO AND KAZUNAGA TANAKA
}

AbStract. We study the nonlinear Schrödinger equations:

$\left(P_{\lambda}\right) \quad-\Delta u+\left(\lambda^{2} a(x)+1\right) u=|u|^{p-1} u, \quad u \in H^{1}\left(\mathbf{R}^{N}\right)$,

where $p>1$ is a subcritical exponent, $a(x)$ is a continuous function satisfying $a(x) \geq 0,0<\liminf _{|x| \rightarrow \infty} a(x) \leq \limsup _{|x| \rightarrow \infty} a(x)<\infty$ and $a^{-1}(0)$ consists of 2 connected bounded smooth components $\Omega_{1}$ and $\Omega_{2}$.

We study the existence of solutions $\left(u_{\lambda}\right)$ of $\left(P_{\lambda}\right)$ which converge to 0 in $\mathbf{R}^{N} \backslash\left(\Omega_{1} \cup \Omega_{2}\right)$ and to a prescribed pair $\left(v_{1}(x), v_{2}(x)\right)$ of solutions of the limit problem:

$$
-\Delta v_{i}+v_{i}=\left|v_{i}\right|^{p-1} v_{i} \quad \text { in } \Omega_{i}
$$

$(i=1,2)$ as $\lambda \rightarrow \infty$.

\section{INTRODUCTION}

In this paper we consider the existence and multiplicity of solutions of the following nonlinear Schrödinger equations:

$\left(P_{\lambda}\right) \quad-\Delta u+\left(\lambda^{2} a(x)+1\right) u=|u|^{p-1} u \quad$ in $\mathbf{R}^{N}, \quad u(x) \in H^{1}\left(\mathbf{R}^{N}\right)$.

Here $p \in\left(1, \frac{N+2}{N-2}\right)$ if $N \geq 3, p \in(1, \infty)$ if $N=1,2$ and $a(x) \in C\left(\mathbf{R}^{N}, \mathbf{R}\right)$ is nonnegative on $\mathbf{R}^{N}$. We consider multiplicity of solutions (including positive and sign-changing solutions) when the parameter $\lambda$ is very large.

For $a(x)$, we assume

(a1) $a(x) \in C\left(\mathbf{R}^{N}, \mathbf{R}\right), a(x) \geq 0$ for all $x \in \mathbf{R}^{N}$ and the potential well $\Omega=$ int $a^{-1}(0)$ is a nonempty bounded open set with smooth boundary $\partial \Omega$ and $a^{-1}(0)=\bar{\Omega}$.

(a2) $0<\liminf _{|x| \rightarrow \infty} a(x) \leq \sup _{x \in \mathbf{R}^{N}} a(x)<\infty$.

When $\lambda$ is large, the potential well $\Omega$ plays important roles and the following Dirichlet problem appears as a limit of $\left(P_{\lambda}\right)$ :

$$
-\Delta u+u=|u|^{p-1} u \quad \text { in } \Omega, \quad u=0 \quad \text { on } \partial \Omega .
$$

Received by the editors June 8, 2005 and, in revised form, October 21, 2005 and May 10, 2007. 2000 Mathematics Subject Classification. Primary 35J60; Secondary 35J20.

Key words and phrases. Nonlinear Schrödinger equations, singular perturbation, critical frequency, sign-changing solutions.

The second author was partially supported by Grant-in-Aid for Scientific Research (C) (2) (No. 17540205) and (B) (2) (No. 20340037) Japan Society for the Promotion of Science.

(C)2009 American Mathematical Society Reverts to public domain 28 years from publication 
We remark that solutions of $\left(P_{\lambda}\right)$ and (1.1) can be characterized as critical points of

$$
\begin{aligned}
& \Psi_{\lambda}(u)=\int_{\mathbf{R}^{N}} \frac{1}{2}\left(|\nabla u|^{2}+\left(\lambda^{2} a(x)+1\right) u^{2}\right)-\frac{1}{p+1}|u|^{p+1} d x: H^{1}\left(\mathbf{R}^{N}\right) \rightarrow \mathbf{R} \\
& \Psi_{\Omega}(u)=\int_{\Omega} \frac{1}{2}\left(|\nabla u|^{2}+u^{2}\right)-\frac{1}{p+1}|u|^{p+1} d x: H_{0}^{1}(\Omega) \rightarrow \mathbf{R}
\end{aligned}
$$

and it is known that (1.3) has an unbounded sequence of critical values (see for example [21]).

Bartsch and Wang [3] and Bartsch, Pankov and Wang [4] (see also [2]) studied such a situation first. Their assumptions on $a(x)$ and nonlinearity are more general, and as a special case of their results we have

(i) There exists a least energy solution $u_{\lambda}(x)$ of $\left(P_{\lambda}\right)$. Moreover $u_{\lambda_{n}}(x)$ converges strongly to a least energy solution of (1.3) after extracting a subsequence $\lambda_{n} \rightarrow \infty$ ([3] $)$.

(ii) When $N \geq 3$ and $p \in\left(1, \frac{N+2}{N-2}\right)$ is close to $\frac{N+2}{N-2}$, there exists at least $\operatorname{cat}(\Omega)$ positive solutions of $\left(P_{\lambda}\right)$ for large $\left.\lambda(\underline{3}]\right)$. Here cat $(\Omega)$ denotes the Lusternik-Schnirelman category of $\Omega$.

(iii) For any $k \in \mathbf{N}$, there exist $k$ pairs of (possibly sign-changing) solutions $\pm u_{1, \lambda}(x), \cdots, \pm u_{k, \lambda}(x)$ of $\left(P_{\lambda}\right)$ for large $\lambda \geq \lambda(k)$. Moreover they converge to distinct solutions $\pm u_{1}(x), \cdots, \pm u_{k}(x)$ of (1.1) after extracting a subsequence $\lambda_{n} \rightarrow \infty$ (4]).

Here we remark that in 3 , 4 they mainly consider the case where $\Omega$ is connected.

In this paper we consider the case where $\Omega$ consists of multiple connected components, $\Omega=\Omega_{1} \cup \Omega_{2} \cup \cdots \cup \Omega_{m}$, and we consider the multiplicity of positive and sign-changing solutions for large $\lambda$.

We studied the multiplicity of positive solutions in our previous paper [16] (see also Cao-Noussair [10]), where it was shown that for any choice of components $\Omega_{k_{1}}$, $\cdots, \Omega_{k_{\ell}}$, there exists a positive solution $u_{\lambda}(x)$ of $\left(P_{\lambda}\right)$ for large $\lambda$ such that after extracting a subsequence $\lambda_{n} \rightarrow \infty$,

$$
u_{\lambda_{n}}(x) \rightarrow \begin{cases}u_{i}(x) & \text { in } \Omega_{i}\left(i \in\left\{k_{1}, \cdots, k_{\ell}\right\}\right), \\ 0 & \text { in } \mathbf{R}^{N} \backslash\left(\Omega_{k_{1}} \cup \cdots \cup \Omega_{k_{\ell}}\right)\end{cases}
$$

strongly in $H^{1}\left(\mathbf{R}^{N}\right)$. Here $u_{i}(x)$ is a least energy solution of

$$
-\Delta u+u=|u|^{p-1} u \quad \text { in } \Omega_{i}, \quad u=0 \quad \text { on } \partial \Omega_{i} .
$$

In particular, $\left(P_{\lambda}\right)$ has at least $2^{m}-1$ positive solutions for large $\lambda$.

We remark that a solution $u_{i}(x)$ of (1.4) is said to be a least energy solution if and only if

$$
\Psi_{i, D}\left(u_{i}\right)=\inf \left\{\Psi_{i, D}(u) ; u(x) \in H_{0}^{1}\left(\Omega_{i}\right) \text { is a nontrivial solution of (1.4) }\right\}
$$

holds. Here $\Psi_{i, D}(u)$ is defined by

$$
\Psi_{i, D}(u)=\int_{\Omega_{i}} \frac{1}{2}\left(|\nabla u|^{2}+u^{2}\right)-\frac{1}{p+1}|u|^{p+1} d x: H_{0}^{1}\left(\Omega_{i}\right) \rightarrow \mathbf{R} .
$$

("D" stands for Dirichlet boundary conditions.) It is natural to ask about the existence of a sequence of solutions of $\left(P_{\lambda}\right)$ converging to solutions of (1.4) in each $\Omega_{i}$, which may not be least energy solutions. 
First we study the multiplicity of sign-changing solutions. For the sake of simplicity, we assume that $\Omega$ consists of 2 components, that is,

$$
\Omega=\Omega_{1} \cup \Omega_{2} \text {. }
$$

In this case we have two limit problems (1.4), which are corresponding to $\Psi_{i, D}$ : $H_{0}^{1}\left(\Omega_{i}\right) \rightarrow \mathbf{R}(i=1,2)$. It is well known that each functional has an unbounded sequence of critical points $\left(u_{j}^{(i)}(x)\right)_{j=1}^{\infty} \subset H_{0}^{1}\left(\Omega_{i}\right)(i=1,2)$. A natural question is to ask, for a given pair $\left(u_{j_{1}}^{(1)}(x), u_{j_{2}}^{(2)}(x)\right)$, whether $\left(P_{\lambda}\right)$ has a solution $u_{\lambda}(x) \in H^{1}\left(\mathbf{R}^{N}\right)$ converging to $u_{j_{i}}^{(i)}(x)$ in $\Omega_{i}$ and to 0 elsewhere. Here we try to give a partial answer to this problem. More precisely, we try to find a solution $u_{\lambda}(x) \in H^{1}\left(\mathbf{R}^{N}\right)$ which converges to $\left(u_{1}^{(1)}(x), u_{j}^{(2)}(x)\right)$ after extracting a subsequence $\lambda_{n} \rightarrow \infty$. Here $u_{1}^{(1)}(x)$ is a mountain pass solution of (1.4) in $\Omega_{1}$ and $u_{j}^{(2)}(x)$ is a minimax solution of (1.4) in $\Omega_{2}$.

To find an unbounded sequence of critical values of a functional $I(u) \in C^{1}(E, \mathbf{R})$ defined on an infinite dimensional Hilbert space $E, \mathbf{Z}_{2}$-symmetry of $I(u)-I( \pm u)=$ $I(u)$ for all $u \in E$ - plays an important role. We remark that $\Psi_{\lambda}(u) \in C^{1}\left(H^{1}\left(\mathbf{R}^{N}\right), \mathbf{R}\right)$ and a functional $\tilde{\Psi}\left(u_{1}, u_{2}\right)=\Psi_{1, D}\left(u_{1}\right)+\Psi_{2, D}\left(u_{2}\right) \in C^{1}\left(H_{0}^{1}\left(\Omega_{1}\right) \times H_{0}^{1}\left(\Omega_{2}\right), \mathbf{R}\right)$, which is corresponding to (1.4) in $\Omega_{1} \cup \Omega_{2}$, have different symmetries. $\Psi_{\lambda}(u)$ is $\mathbf{Z}_{2}$-symmetric and $\tilde{\Psi}\left(u_{1}, u_{2}\right)$ is $\left(\mathbf{Z}_{2}\right)^{2}$-symmetric; that is,

$$
\begin{aligned}
& \Psi_{\lambda}(s u)=\Psi_{\lambda}(u) \quad \text { for all } s \in \mathbf{Z}_{2}=\{-1,1\}, u \in H^{1}\left(\mathbf{R}^{N}\right), \\
& \tilde{\Psi}\left(s_{1} u_{1}, s_{2} u_{2}\right)=\tilde{\Psi}\left(u_{1}, u_{2}\right) \text { for all } s_{1}, s_{2} \in \mathbf{Z}_{2},\left(u_{1}, u_{2}\right) \in H_{0}^{1}\left(\Omega_{1}\right) \times H_{0}^{1}\left(\Omega_{2}\right) .
\end{aligned}
$$

Note that $\mathbf{Z}_{2}$-action on $\Psi_{\lambda}(u)$ is corresponding to the following $\mathbf{Z}_{2}$-action on $\tilde{\Psi}\left(u_{1}, u_{2}\right)$ :

$$
\tilde{\Psi}\left(s u_{1}, s u_{2}\right)=\tilde{\Psi}\left(u_{1}, u_{2}\right) \quad \text { for all } s \in\{-1,1\},\left(u_{1}, u_{2}\right) \in H_{0}^{1}\left(\Omega_{1}\right) \times H_{0}^{1}\left(\Omega_{2}\right),
$$

and there are no symmetries of $\Psi_{\lambda}(u)$ corresponding to the $\mathbf{Z}_{2}$-symmetry of $\tilde{\Psi}\left(u_{1}, u_{2}\right)$ :

$$
\tilde{\Psi}\left(u_{1}, \pm u_{2}\right)=\tilde{\Psi}\left(u_{1}, u_{2}\right) .
$$

We also remark that solutions $\left(u_{1}^{(1)}(x), u_{j}^{(2)}(x)\right)$ are obtained using the group action (1.6). Thus to construct solutions $u_{\lambda}(x)$ converging to $\left(u_{1}^{(1)}(x), u_{j}^{(2)}(x)\right)$ we need to develop a kind of perturbation theory from symmetries, and in this paper we use ideas from Ambrosetti [1, Bahri-Berestycki [5], Struwe [23] and Rabinowitz 20. (see also Bahri-Lions [6], Tanaka 25, and Bolle [8]). In [1, 5, 6, 20, 23, 25, perturbation theories are developed for

$$
-\Delta u=|u|^{p-1} u+f(x) \quad \text { in } \Omega, \quad u=0 \quad \text { on } \partial \Omega,
$$

where $\Omega \subset \mathbf{R}^{N}$ is a bounded domain. They successfully showed the existence of an unbounded sequence of solutions for all $f(x) \in L^{2}(\Omega)$ for a certain range of $p$.

To state our result, we need some notation: we set

$$
\Sigma_{i, D}=\left\{u \in H_{0}^{1}\left(\Omega_{i}\right) ;\|u\|_{H_{0}^{1}\left(\Omega_{i}\right)}=1\right\}
$$

and consider the constraint functional

$$
J_{i, D}(v)=\max _{t>0} \Psi_{i, D}(t v): \Sigma_{i, D} \rightarrow \mathbf{R} .
$$


We define

$$
\begin{aligned}
c_{\min }^{1, D} & =\inf _{v \in \Sigma_{1, D}} J_{1, D}(v), \\
b_{n}^{2, D} & =\inf _{\gamma \in \Gamma_{n}^{2, D}} \max _{\theta \in S^{n}} J_{2, D}(\gamma(\theta)),
\end{aligned}
$$

where $S^{n}=\left\{\theta \in \mathbf{R}^{n+1} ;|\theta|=1\right\}$ and

$$
\Gamma_{n}^{2, D}=\left\{\gamma \in C\left(S^{n}, \Sigma_{2, D}\right) ; \gamma(-\theta)=-\gamma(\theta) \text { for all } \theta \in S^{n}\right\} .
$$

We will observe that $c_{\text {min }}^{1, D}$ and $b_{n}^{2, D}$ are critical values of $\Psi_{1, D}(u), \Psi_{2, D}(u)$ and

$$
b_{0}^{2, D} \leq b_{1}^{2, D} \leq b_{2}^{2, D} \leq \cdots \leq b_{n}^{2, D} \leq b_{n+1}^{2, D} \leq \cdots, \quad b_{n}^{2, D} \rightarrow \infty(n \rightarrow \infty) .
$$

In particular, there exists a sequence $n(1)<n(2)<\cdots<n(k)<n(k+1)<\cdots$ such that $b_{n(k)}^{2, D}<b_{n(k)+1}^{2, D}$. We also define another set of minimax values by

$$
c_{k}^{2, D}=\inf _{\sigma \in \Lambda_{k}} \max _{\theta \in S_{+}^{n(k)+1}} J_{2, D}(\sigma(\theta))
$$

where $S_{+}^{n(k)+1}=\left\{\theta=\left(\theta_{1}, \cdots, \theta_{n(k)+1}, \theta_{n(k)+2}\right) ; \theta \in S^{n(k)+1}, \theta_{n(k)+2} \geq 0\right\}$ and

$$
\Lambda_{k}=\left\{\sigma \in C\left(S_{+}^{n(k)+1}, \Sigma_{2, D}\right) ;\left.\sigma\right|_{S^{n(k)}} \in \Gamma_{n(k)}^{2, D}, \inf _{\theta \in S^{n(k)}} \Psi_{2, D}(\sigma(\theta))<b_{n(k)}^{2, D}+\delta_{k}\right\} .
$$

Here $\delta_{k}=\frac{1}{4}\left(b_{n(k)+1}^{2, D}-b_{n(k)}^{2, D}\right)>0$. We can also see that $c_{k}^{2, D}$ is a critical value of $\Psi_{2, D}(u)$ and $c_{k}^{2, D} \rightarrow \infty$ as $k \rightarrow \infty$.

Now we can give our main result.

Theorem 1.1. Assume (a1)-(a2) and (1.5). Then for any $k \in \mathbf{N}$ there exists $\lambda_{1}(k) \geq 1$ such that for any $\lambda \geq \lambda_{1}(k),\left(P_{\lambda}\right)$ has a solution $u_{\lambda}(x)$ such that

$$
\Psi_{\lambda}\left(u_{\lambda}\right) \rightarrow c_{\min }^{1, D}+c_{k}^{2, D} \quad \text { as } \lambda \rightarrow \infty
$$

(ii) For any given sequence $\lambda_{n} \rightarrow \infty$, we can extract a subsequence $\lambda_{n_{\ell}} \rightarrow \infty$ such that $u_{\lambda_{n_{\ell}}}$ converges to a function $u(x)$ strongly in $H^{1}\left(\mathbf{R}^{N}\right)$. Moreover we have

$$
\begin{aligned}
& u(x) \text { satisfies (1.4) in } \Omega_{1} \cup \Omega_{2}, \\
& \left.u\right|_{\mathbf{R}^{N} \backslash\left(\Omega_{1} \cup \Omega_{2}\right)} \equiv 0, \\
& u(x)>0 \quad \text { in } \Omega_{1}, \\
& \Psi_{1, D}\left(\left.u\right|_{\Omega_{1}}\right)=c_{\text {min }}^{1, D}, \quad \Psi_{2, D}\left(\left.u\right|_{\Omega_{2}}\right)=c_{k}^{2, D} .
\end{aligned}
$$

When $N=1$, we have a stronger result. Namely,

Theorem 1.2. Assume $N=1$ and $\Omega_{i}=\left(a_{i}, b_{i}\right)(i=1,2)$, where $a_{1}<b_{1}<a_{2}<$ $b_{2}$. Then for any solution $v_{1}(x)$ of (1.1) in $\Omega_{1}$ and for any solution $v_{2}(x)$ of (1.1) in $\Omega_{2}$, there exists a solution $u_{\lambda}(x)$ for large $\lambda$ such that

$$
u_{\lambda}(x) \rightarrow \begin{cases}v_{1}(x) & \text { in } \Omega_{1}, \\ v_{2}(x) & \text { in } \Omega_{2}, \\ 0 & \text { in } \mathbf{R} \backslash\left(\Omega_{1} \cup \Omega_{2}\right)\end{cases}
$$

strongly in $H^{1}(\mathbf{R})$ as $\lambda \rightarrow \infty$.

Next we deal with positive solutions. Our result is influenced by the result [3]. 
Theorem 1.3. Assume (a1)-(a2), (1.5) and $N \geq 3$. Then there exists a $p_{1} \in$ $\left(1, \frac{N+2}{N-2}\right)$ and $\lambda_{1}(p) \geq 1$ depending on $p \in\left(1, \frac{N+2}{N-2}\right)$ such that for $p \in\left(p_{1}, \frac{N+2}{N-2}\right)$ and $\lambda \geq \lambda_{1}(p),\left(P_{\lambda}\right)$ possesses at least $\operatorname{cat}\left(\Omega_{1}\right)+\operatorname{cat}\left(\Omega_{2}\right)+\operatorname{cat}\left(\Omega_{1} \times \Omega_{2}\right)$ positive solutions.

Remark 1.4. Since cat $\left(\Omega_{1} \cup \Omega_{2}\right)=\operatorname{cat}\left(\Omega_{1}\right)+\operatorname{cat}\left(\Omega_{2}\right)$, the argument of Bartsch-Wang [3] ensures cat $\left(\Omega_{1}\right)+\operatorname{cat}\left(\Omega_{2}\right)$ positive solutions, which converge to a positive solution of (1.3) in one of the components and to 0 elsewhere after extracting a subsequence $\lambda_{n} \rightarrow \infty$. We remark that our Theorem 1.3 ensures additional cat $\left(\Omega_{1} \times \Omega_{2}\right)$ positive solutions. We can also observe that these solutions converge to positive solutions in both components $\Omega_{1}, \Omega_{2}$.

Similarly, we can deal with sign-changing solutions which converge to positive solutions in $\Omega_{1}$ and negative solutions in $\Omega_{2}$.

Theorem 1.5. Assume (a1)-(a2), (1.5) and $N \geq 3$. Then there exists a $p_{1} \in$ $\left(1, \frac{N+2}{N-2}\right)$ and $\lambda_{1}(p) \geq 1$ depending on $p \in\left(1, \frac{N+2}{N-2}\right)$ such that for $p \in\left(p_{1}, \frac{N+2}{N-2}\right)$ and $\lambda \geq \lambda_{1}(p),\left(P_{\lambda}\right)$ possesses at least cat $\left(\Omega_{1} \times \Omega_{2}\right)$ sign-changing solutions. Moreover, after extracting a subsequence $\lambda_{n} \rightarrow \infty$, they converge to positive solutions of (1.4) in $\Omega_{1}$ and negative solutions of (1.4) in $\Omega_{2}$.

In the following sections we will give proofs to our theorems. We will take an approach different from that of Ding and Tanaka [16]. In Section 22 we introduce a reduced functional $J_{\lambda}\left(v_{1}, v_{2}\right)$, which is defined on an infinite dimensional torus $\Sigma_{1, \lambda} \times \Sigma_{2, \lambda}-\Sigma_{1, \lambda}$ and $\Sigma_{2, \lambda}$ will be defined in Section 2 (d). We will observe that for large $\lambda$ our reduced functional $J_{\lambda}\left(v_{1}, v_{2}\right)$ can be approximated by $J_{1, \lambda}\left(v_{1}\right)+J_{2, \lambda}\left(v_{2}\right)-J_{1, \lambda}\left(v_{1}\right)$ and $J_{2, \lambda}\left(v_{2}\right)$ are defined as in (1.7) - in a suitable sense and that it enables us to develop a perturbation theory. In Sections 2 , 3 we develop a perturbation theory for $J_{\lambda} \in C^{1}\left(\Sigma_{1, \lambda} \times \Sigma_{2, \lambda}, \mathbf{R}\right)$. The subsolution estimate for Schrödinger operators (cf. Simon [22]) and a perturbation argument under the influence of 1, 15, 20, 23 play essential roles. Section 4 is devoted to showing Theorem 1.1. Isolatedness of critical values of $\Psi_{1, D}\left(v_{1}\right)$ corresponding to positive solutions is important, and we will construct a deformation flow for $J_{\lambda} \in$ $C^{1}\left(\Sigma_{1, \lambda} \times \Sigma_{2, \lambda}, \mathbf{R}\right)$, under which the level set $\left\{v_{1} \in \Sigma_{1, \lambda} ; J_{1, \lambda}\left(v_{1}\right) \leq c\right\}$ for the unperturbed functional $J_{1, \lambda}\left(v_{1}\right)$ is invariant for a certain range of $c$. In Section 5 we will give a proof to Theorem 1.2 via Nehari type methods (cf. del Pino, Felmer and Tanaka [15]). In Section [6, we give a proof to our Theorems 1.3, 1.5. Here an estimate of the category of the level set $\left\{\left(v_{1}, v_{2}\right) \in \Sigma_{1, \lambda} \times \Sigma_{2, \lambda} ; J_{\lambda}\left(v_{1}, v_{2}\right) \leq c\right\}$ is essential. Sections 79 are devoted to proving various propositions and lemmas which are used in previous sections without proofs.

\section{Functional SETting AND VARiational Formulation}

(a) Functional setting. To find critical points of $\Psi_{\lambda}(u)$ in $H^{1}\left(\mathbf{R}^{N}\right)$, we introduce some notation: for an open set $O \subset \mathbf{R}^{N}$ and $\lambda \geq 1$ we write

$$
\begin{aligned}
\langle u, v\rangle_{\lambda, O} & =\int_{O} \nabla u \nabla v+\left(\lambda^{2} a(x)+1\right) u v d x \quad \text { for } u, v \in H^{1}(O), \\
\|u\|_{\lambda, O} & =\sqrt{\langle u, u\rangle_{\lambda, O}} \quad \text { for } u \in H^{1}(O), \\
\|f\|_{\lambda, O}^{*} & =\sup _{u \in H^{1}(O),\|u\|_{\lambda, O} \leq 1}|f(u)| \quad \text { for } f \in\left(H^{1}(O)\right)^{*} .
\end{aligned}
$$


By the assumption $\|\cdot\|_{\lambda, O}$ is equivalent to the standard $H^{1}$-norm

$$
\|u\|_{H^{1}(O)}^{2}=\int_{O}|\nabla u|^{2}+u^{2} d x
$$

and we have

$$
\|u\|_{H^{1}(O)} \leq\|u\|_{\lambda, O} \quad \text { for all } u \in H^{1}(O) \text { and } \lambda \geq 1
$$

We also use

$$
\begin{aligned}
\|u\|_{L^{r}(O)}^{r} & =\int_{O}|u|^{r} d x \quad \text { for } 1 \leq r<\infty, \\
\|u\|_{L^{\infty}(O)} & =\operatorname{esssup}_{x \in O}|u(x)| .
\end{aligned}
$$

For each $i \in\{1,2\}$, we choose bounded open subsets $\Omega_{i}^{\prime}, \Omega_{i}^{\prime \prime}$ with smooth boundaries such that

$$
\Omega_{i} \subset \subset \Omega_{i}^{\prime} \subset \subset \Omega_{i}^{\prime \prime}(i=1,2), \quad \overline{\Omega_{1}^{\prime \prime}} \cap \overline{\Omega_{2}^{\prime \prime}}=\emptyset .
$$

Here and in what follows we write $A \subset \subset B$ if $\bar{A} \subset \operatorname{int}(B)$ holds. We will use the following lemma repeatedly with $O=\mathbf{R}^{N} \backslash\left(\Omega_{1}^{\prime} \cup \Omega_{2}^{\prime}\right), O=\left(\Omega_{1}^{\prime \prime} \backslash \Omega_{1}^{\prime}\right) \cup\left(\Omega_{2}^{\prime \prime} \backslash \Omega_{2}^{\prime}\right)$, etc.

Lemma 2.1. For a domain $O \subset \mathbf{R}^{N}$ with $\bar{O} \cap\left(\overline{\Omega_{1}} \cup \overline{\Omega_{2}}\right)=\emptyset$ and $r \in\left[2, \frac{2 N}{N-2}\right)$ $(N \geq 3)$ and $r \in[2, \infty)(N=1,2)$, there exists a constant $C_{r}(O, \lambda)$ satisfying $C_{r}(O, \lambda) \rightarrow 0$ as $\lambda \rightarrow \infty$ such that

$$
\|u\|_{L^{r}(O)} \leq C_{r}(O, \lambda)\|u\|_{\lambda, O} \quad \text { for all } u \in H^{1}(O) .
$$

Proof. Let $a_{\infty}(O)=\inf _{x \in O} a(x)>0$. Then we have

$$
\|u\|_{L^{2}(O)} \leq \frac{1}{\sqrt{a_{\infty}(O)} \lambda}\|u\|_{\lambda, O} \quad \text { for all } u \in H^{1}(O) .
$$

On the other hand, by (2.1) we have

$$
\|u\|_{L^{\frac{2 N}{N-2}}(O)} \leq C\|u\|_{\lambda, O} \quad(N \geq 3), \quad\|u\|_{L^{q}(O)} \leq C_{q}\|u\|_{\lambda, O} \quad(N=1,2),
$$

where $q>r$. Thus by the interpolation we have the conclusion of Lemma 2.1.

(b) Modification of the nonlinearity $|u|^{p-1} u$. To find solutions with asymptotic behaviors as in Theorems 1.1, 1.3, and 1.5 we take the local mountain pass approach due to del Pino and Felmer [14]. We choose an odd function $f(\xi) \in C^{1}(\mathbf{R}, \mathbf{R})$ such that for some $0<\ell_{1}<\ell_{2}$

$$
\begin{aligned}
f(\xi) & =|\xi|^{p-1} \xi \quad \text { for }|\xi| \leq \ell_{1}, \\
f(\xi) & =\frac{1}{2} \xi \text { for }|\xi| \geq \ell_{2}, \\
0 & \leq f^{\prime}(\xi) \leq \frac{2}{3} \quad \text { for all } \xi \in \mathbf{R} .
\end{aligned}
$$

We set

$$
g(x, \xi)= \begin{cases}|\xi|^{p-1} \xi & \text { if } x \in \Omega_{1}^{\prime} \cup \Omega_{2}^{\prime}, \\ f(\xi) & \text { if } x \in \mathbf{R}^{N} \backslash\left(\Omega_{1}^{\prime} \cup \Omega_{2}^{\prime}\right) .\end{cases}
$$

We also use the notation

$$
G(x, \xi)=\int_{0}^{\xi} g(x, s) d s
$$


In what follows we will try to find critical points of

$$
\begin{aligned}
\Phi_{\lambda}(u) & =\frac{1}{2} \int_{\mathbf{R}^{N}}|\nabla u|^{2}+\left(\lambda^{2} a(x)+1\right) u^{2} d x-\int_{\mathbf{R}^{N}} G(x, u) d x \\
& =\frac{1}{2}\|u\|_{\lambda, \mathbf{R}^{N}}^{2}-\int_{\mathbf{R}^{N}} G(x, u) d x .
\end{aligned}
$$

First we have

Proposition 2.2. For $\lambda \geq 1, \Phi_{\lambda}(u) \in C^{2}\left(H^{1}\left(\mathbf{R}^{N}\right)\right.$, $\left.\mathbf{R}\right)$ satisfies $(P S)_{c}$ condition for all $c \in \mathbf{R}$; that is, any sequence $\left(u_{n}\right)_{n=1}^{\infty} \subset H^{1}\left(\mathbf{R}^{N}\right)$ satisfying for $c \in \mathbf{R}$

$$
\Phi_{\lambda}\left(u_{n}\right) \rightarrow c, \quad \Phi_{\lambda}^{\prime}\left(u_{n}\right) \rightarrow 0 \quad \text { strongly in } H^{-1}\left(\mathbf{R}^{N}\right)
$$

has a strongly convergent subsequence in $H^{1}\left(\mathbf{R}^{N}\right)$.

Proposition 2.3. Suppose that $\left(u_{\lambda}(x)\right)_{\lambda \geq \lambda_{0}}$ is a family of critical points of $\Phi_{\lambda}(u)$ and assume that there exist constants $m, M>0$ independent of $\lambda$ such that

$$
m \leq \Phi_{\lambda}\left(u_{\lambda}\right) \leq M \quad \text { for all } \lambda \geq \lambda_{0} .
$$

Then we have

(i) $\left(\frac{1}{2}-\frac{1}{p+1}\right)^{-1} m \leq\left\|u_{\lambda}\right\|_{\lambda, \mathbf{R}^{N}}^{2} \leq\left(\frac{1}{2}-\frac{1}{p+1}\right)^{-1} M$ for all $\lambda \geq \lambda_{0}$.

(ii) There exists $\lambda(M) \geq \lambda_{0}$ such that for $\lambda \geq \lambda(M), u_{\lambda}(x)$ satisfies

$$
\left|u_{\lambda}(x)\right| \leq \ell_{1} \quad \text { for } x \in \mathbf{R}^{N} \backslash\left(\Omega_{1}^{\prime} \cup \Omega_{2}^{\prime}\right) .
$$

In particular, $g\left(x, u_{\lambda}(x)\right)=\left|u_{\lambda}(x)\right|^{p-1} u_{\lambda}(x)$ holds in $\mathbf{R}^{N}$ and $u_{\lambda}(x)$ is a solution of the original problem $\left(P_{\lambda}\right)$.

(iii) After extracting a subsequence $\lambda_{n} \rightarrow \infty$, there exists $u \in H^{1}\left(\mathbf{R}^{N}\right)$ such that

$$
\left\|u_{\lambda_{n}}-u\right\|_{\lambda_{n}, \mathbf{R}^{N}} \rightarrow 0 \text { as } n \rightarrow \infty \text {. }
$$

Moreover, $u(x)$ satisfies $u(x) \equiv 0$ in $\mathbf{R}^{N} \backslash\left(\Omega_{1} \cup \Omega_{2}\right)$ and

$$
\begin{aligned}
-\Delta u+u & =|u|^{p-1} u \quad \text { in } \Omega_{i}, \\
u & =0 \quad \text { on } \partial \Omega_{i}
\end{aligned}
$$

for $i=1,2$. It also holds that $\Phi_{\lambda_{n}}\left(u_{\lambda_{n}}\right) \rightarrow \Psi_{1, D}\left(\left.u\right|_{\Omega_{1}}\right)+\Psi_{2, D}\left(\left.u\right|_{\Omega_{2}}\right)$ as $n \rightarrow \infty$.

Proofs of Propositions 2.2, 2.3 can be given as in Section 2 of our previous paper [16.

(c) Reduction to a problem on $H^{1}\left(\Omega_{1}^{\prime} \cup \Omega_{2}^{\prime}\right)$. To find critical points of $\Phi_{\lambda}(u)$, we introduce modified problems. First, we reduce our problem to a problem on $H^{1}\left(\Omega_{1}^{\prime} \cup \Omega_{2}^{\prime}\right)$. For a given $u \in H^{1}\left(\Omega_{1}^{\prime} \cup \Omega_{2}^{\prime}\right)$ we define

$$
I_{\lambda}(u)=\inf _{w \in A_{u}} \Phi_{\lambda}(w),
$$

where $A_{u}=\left\{w \in H^{1}\left(\mathbf{R}^{N}\right) ; w=u\right.$ on $\left.\Omega_{1}^{\prime} \cup \Omega_{2}^{\prime}\right\}$. First we have

Proposition 2.4. For any $u \in H^{1}\left(\Omega_{1}^{\prime} \cup \Omega_{2}^{\prime}\right)$ and $\lambda \geq 1$ there exists a unique minimizer $w_{\lambda}(u) \in A_{u}$. Moreover, $w_{\lambda}(u)$ satisfies

(i) $w_{\lambda}(u)$ is nondegenerate in the following sense:

$$
\Phi_{\lambda}^{\prime \prime}\left(w_{\lambda}(u)\right)(h, h) \geq \frac{1}{3}\|h\|_{\lambda, \mathbf{R}^{N}}^{2} \backslash\left(\Omega_{1}^{\prime} \cup \Omega_{2}^{\prime}\right) \quad \text { for all } h \in H_{0}^{1}\left(\mathbf{R}^{N} \backslash\left(\Omega_{1}^{\prime} \cup \Omega_{2}^{\prime}\right)\right) \text {. }
$$

(ii) $H^{1}\left(\Omega_{1}^{\prime} \cup \Omega_{2}^{\prime}\right) \rightarrow H^{1}\left(\mathbf{R}^{N}\right) ; u \mapsto w_{\lambda}(u)$ is of class $C^{1}$. 
(iii) $I_{\lambda}(u)=\Phi_{\lambda}\left(w_{\lambda}(u)\right): H^{1}\left(\Omega_{1}^{\prime} \cup \Omega_{2}^{\prime}\right) \rightarrow \mathbf{R}$ is of class $C^{2}$.

(iv) $u \in H^{1}\left(\Omega_{1}^{\prime} \cup \Omega_{2}^{\prime}\right)$ is a critical point of $I_{\lambda}(u)$ if and only if $w_{\lambda}(u)$ is a critical point of $\Phi_{\lambda}(u)$.

(v) $I_{\lambda}(u)$ satisfies $(P S)_{c}$ condition for all $c \in \mathbf{R}$.

For a proof of Proposition 2.4, we choose sets $\Omega_{1}^{(1)}, \Omega_{2}^{(1)}$ such that $\Omega_{i} \subset \subset \Omega_{i}^{(1)}$ $\subset \subset \Omega_{i}^{\prime}$ for $i=1,2$. We need the following lemma.

Lemma 2.5. There exists a linear operator $P: H^{1}\left(\Omega_{1}^{\prime} \cup \Omega_{2}^{\prime}\right) \rightarrow H^{1}\left(\mathbf{R}^{N}\right)$ with the following properties: for $\lambda \geq 1$ and $u \in H^{1}\left(\Omega_{1}^{\prime} \cup \Omega_{2}^{\prime}\right)$

$$
\begin{aligned}
& (P u)(x)=u(x) \quad \text { for all } x \in \Omega_{1}^{\prime} \cup \Omega_{2}^{\prime}, \\
& \operatorname{supp} P u \subset \Omega_{1}^{\prime \prime} \cup \Omega_{2}^{\prime \prime}, \\
& \|P u\|_{H^{1}\left(D_{\text {out }}\right)} \leq C_{1}\|u\|_{H^{1}\left(D_{\text {in }}\right)}, \\
& \|P u\|_{L^{2}\left(D_{\text {out }}\right)} \leq C_{1}\|u\|_{L^{2}\left(D_{\text {in }}\right)}, \\
& \|P u\|_{L^{p+1}\left(D_{\text {out }}\right)} \leq C_{1}\|u\|_{L^{p+1}\left(D_{\text {in }}\right)}, \\
& \|P u\|_{\lambda, D_{\text {out }}} \leq C_{1}\|u\|_{\lambda, D_{\text {in }}}, \\
& \|P u\|_{\lambda, \mathbf{R}^{N}} \leq C_{1}\|u\|_{\lambda, \Omega_{1}^{\prime} \cup \Omega_{2}^{\prime} .} .
\end{aligned}
$$

Here we use notation

$$
D_{\text {out }}=\left(\Omega_{1}^{\prime \prime} \backslash \Omega_{1}^{\prime}\right) \cup\left(\Omega_{2}^{\prime \prime} \backslash \Omega_{2}^{\prime}\right), \quad D_{\text {in }}=\left(\Omega_{1}^{\prime} \backslash \Omega_{1}^{(1)}\right) \cup\left(\Omega_{2}^{\prime} \backslash \Omega_{2}^{(1)}\right),
$$

and $C_{1}>0$ is a constant independent of $\lambda \geq 1$ and $u \in H^{1}\left(\Omega_{1}^{\prime} \cup \Omega_{2}^{\prime}\right)$.

Proof. (2.6)-(2.8) are rather standard. For (2.9) we have from (2.6)-(2.7)

$$
\begin{aligned}
\|P u\|_{\lambda, D_{\text {out }}}^{2} & \leq\|P u\|_{H^{1}\left(D_{\text {out }}\right)}^{2}+\lambda^{2}\left(\sup _{x \in D_{\text {out }}} a(x)\right)\|P u\|_{L^{2}\left(D_{\text {out }}\right)}^{2} \\
& \leq C_{1}^{2}\|u\|_{H^{1}\left(D_{\text {in }}\right)}^{2}+C_{1}^{2} \lambda^{2}\left(\sup _{x \in D_{\text {out }}} a(x)\right)\|u\|_{L^{2}\left(D_{\text {in }}\right)}^{2} \\
& \leq C_{1}^{2}\|u\|_{H^{1}\left(D_{\text {in }}\right)}^{2}+C_{1}^{2} \frac{\sup _{x \in D_{\text {out }}} a(x)}{\inf _{x \in D_{\text {in }}} a(x)} \int_{D_{\text {in }}} \lambda^{2} a(x) u^{2} d x \\
& \leq C_{1}^{2}\left(1+\frac{\sup _{x \in D_{\text {out }}} a(x)}{\inf _{x \in D_{\text {in }}} a(x)}\right)\|u\|_{\lambda, D_{\text {in }}}^{2} .
\end{aligned}
$$

Thus we get (2.9). Property (2.10) also follows from (2.9).

Proof of Proposition 2.4. First we show the existence and the uniqueness of a minimizer of $\Phi_{\lambda}(w)$ in $A_{u}$. It suffices to show that $A_{u} \rightarrow \mathbf{R} ; w \mapsto \Phi_{\lambda}(w)$ is coercive and strictly convex. Since $w=u$ on $\Omega_{1}^{\prime} \cup \Omega_{2}^{\prime}$ for all $w \in A_{u}$, we need to show

$$
A_{u} \rightarrow \mathbf{R} ; w \mapsto \frac{1}{2}\|w\|_{\lambda, \mathbf{R}^{N} \backslash\left(\Omega_{1}^{\prime} \cup \Omega_{2}^{\prime}\right)}^{2}-\int_{\mathbf{R}^{N} \backslash\left(\Omega_{1}^{\prime} \cup \Omega_{2}^{\prime}\right)} G(x, w) d x
$$

is coercive and strictly convex. We note that

$$
|g(x, \xi)|=|f(\xi)| \leq \frac{2}{3}|\xi|, \quad G(x, \xi) \leq \frac{1}{3}|\xi|^{2}
$$


for all $x \in \mathbf{R}^{N} \backslash\left(\Omega_{1}^{\prime} \cup \Omega_{2}^{\prime}\right)$ and $\xi \in \mathbf{R}$. We can observe that

$$
\begin{aligned}
& \frac{1}{2}\|w\|_{\lambda, \mathbf{R}^{N} \backslash\left(\Omega_{1}^{\prime} \cup \Omega_{2}^{\prime}\right)}^{2}-\int_{\mathbf{R}^{N} \backslash\left(\Omega_{1}^{\prime} \cup \Omega_{2}^{\prime}\right)} G(x, w) d x \\
\geq & \frac{1}{2}\|w\|_{\lambda, \mathbf{R}^{N} \backslash\left(\Omega_{1}^{\prime} \cup \Omega_{2}^{\prime}\right)}^{2}-\frac{1}{3}\|w\|_{L^{2}\left(\mathbf{R}^{N} \backslash\left(\Omega_{1}^{\prime} \cup \Omega_{2}^{\prime}\right)\right)}^{2} \geq \frac{1}{6}\|w\|_{\lambda, \mathbf{R}^{N} \backslash\left(\Omega_{1}^{\prime} \cup \Omega_{2}^{\prime}\right)}^{2},
\end{aligned}
$$

and we get coerciveness of $w \mapsto \Phi_{\lambda}(w)$. On the other hand, since $A_{u}=\{P u+h ; h \in$ $\left.H_{0}^{1}\left(\mathbf{R}^{N} \backslash\left(\Omega_{1}^{\prime} \cup \Omega_{2}^{\prime}\right)\right)\right\}$, for strict convexity of $\Phi_{\lambda}(w)$ on $A_{u}$ it suffices to show that $\Phi_{\lambda}^{\prime \prime}(w)(h, h) \geq \frac{1}{3}\|h\|_{\lambda, \mathbf{R}^{N} \backslash\left(\Omega_{1}^{\prime} \cup \Omega_{2}^{\prime}\right)}^{2}$ for all $w \in H^{1}\left(\mathbf{R}^{N}\right)$ and $h \in H_{0}^{1}\left(\mathbf{R}^{N} \backslash\left(\Omega_{1}^{\prime} \cup \Omega_{2}^{\prime}\right)\right)$. Indeed, we have by (2.2),

$$
\begin{aligned}
\Phi_{\lambda}^{\prime \prime}(w)(h, h) & =\|h\|_{\lambda, \mathbf{R}^{N} \backslash\left(\Omega_{1}^{\prime} \cup \Omega_{2}^{\prime}\right)}^{2}-\int_{\mathbf{R}^{N} \backslash\left(\Omega_{1}^{\prime} \cup \Omega_{2}^{\prime}\right)} g^{\prime}(x, w) h^{2} d x \\
& \geq\|h\|_{\lambda, \mathbf{R}^{N} \backslash\left(\Omega_{1}^{\prime} \cup \Omega_{2}^{\prime}\right)}^{2}-\frac{2}{3}\|h\|_{L^{2}\left(\mathbf{R}^{N} \backslash\left(\Omega_{1}^{\prime} \cup \Omega_{2}^{\prime}\right)\right)}^{2} \geq \frac{1}{3}\|h\|_{\lambda, \mathbf{R}^{N} \backslash\left(\Omega_{1}^{\prime} \cup \Omega_{2}^{\prime}\right)}^{2} .
\end{aligned}
$$

Thus $w \mapsto \Phi_{\lambda}(w)$ is strictly convex. Therefore for any $u \in H^{1}\left(\Omega_{1}^{\prime} \cup \Omega_{2}^{\prime}\right)$ there exists a unique minimizer $w_{\lambda}(u) \in A_{u}$ of $\Phi_{\lambda}(w)$. (i) is also proved.

Next we show (ii). We consider the following mapping:

$$
\begin{aligned}
F: \quad & H^{1}\left(\Omega_{1}^{\prime} \cup \Omega_{2}^{\prime}\right) \times H_{0}^{1}\left(\mathbf{R}^{N} \backslash\left(\Omega_{1}^{\prime} \cup \Omega_{2}^{\prime}\right)\right) \rightarrow H^{-1}\left(\mathbf{R}^{N} \backslash\left(\Omega_{1}^{\prime} \cup \Omega_{2}^{\prime}\right)\right), \\
& \left.(u, \varphi) \mapsto \Phi_{\lambda}^{\prime}(P u+\varphi)\right|_{H_{0}^{1}\left(\mathbf{R}^{N} \backslash\left(\Omega_{1}^{\prime} \cup \Omega_{2}^{\prime}\right)\right) .}
\end{aligned}
$$

By the convexity of $\varphi \mapsto \Phi_{\lambda}(P u+\varphi), F(u, \varphi)=0$ holds if and only if $\varphi=\varphi_{\lambda}(u)$, where $\varphi_{\lambda}(u): H^{1}\left(\Omega_{1}^{\prime} \cup \Omega_{2}^{\prime}\right) \rightarrow H_{0}^{1}\left(\mathbf{R}^{N} \backslash\left(\Omega_{1}^{\prime} \cup \Omega_{2}^{\prime}\right)\right)$ is defined by $\varphi_{\lambda}(u)=w_{\lambda}(u)-$ $P u$. We have for $h_{1}, h_{2} \in H_{0}^{1}\left(\mathbf{R}^{N} \backslash\left(\Omega_{1}^{\prime} \cup \Omega_{2}^{\prime}\right)\right)$

$\left\langle F_{\varphi}(u, \varphi) h_{1}, h_{2}\right\rangle=\int_{\mathbf{R}^{N} \backslash\left(\Omega_{1}^{\prime} \cup \Omega_{2}^{\prime}\right)} \nabla h_{1} \nabla h_{2}+\left(\lambda^{2} a(x)+1\right) h_{1} h_{2}-g^{\prime}(x, P u+\varphi) h_{1} h_{2} d x$.

By (2.2), we can observe that

$$
F_{\varphi}(u, \varphi): H_{0}^{1}\left(\mathbf{R}^{N} \backslash\left(\Omega_{1}^{\prime} \cup \Omega_{2}^{\prime}\right)\right) \rightarrow H^{-1}\left(\mathbf{R}^{N} \backslash\left(\Omega_{1}^{\prime} \cup \Omega_{2}^{\prime}\right)\right)
$$

is invertible for all $(u, \varphi) \in H^{1}\left(\Omega_{1}^{\prime} \cup \Omega_{2}^{\prime}\right) \times H_{0}^{1}\left(\mathbf{R}^{N} \backslash\left(\Omega_{1}^{\prime} \cup \Omega_{2}^{\prime}\right)\right)$. Thus, by the implicit function theorem, we can observe $u \mapsto \varphi_{\lambda}(u)$ is of class $C^{1}$. That is, $u \mapsto w_{\lambda}(u)=P u+\varphi_{\lambda}(u)$ is of class $C^{1}$.

For (iii), we remark that $I_{\lambda}(u)=\Phi_{\lambda}\left(w_{\lambda}(u)\right)=\Phi_{\lambda}\left(P u+\varphi_{\lambda}(u)\right)$ holds. We also remark that

$$
\Phi_{\lambda}^{\prime}\left(P u+\varphi_{\lambda}(u)\right) h=0 \quad \text { for all } h \in H_{0}^{1}\left(\mathbf{R}^{N} \backslash\left(\Omega_{1}^{\prime} \cup \Omega_{2}^{\prime}\right)\right) .
$$

Noting $\varphi_{\lambda}^{\prime}(u) \zeta \in H_{0}^{1}\left(\mathbf{R}^{N} \backslash\left(\Omega_{1}^{\prime} \cup \Omega_{2}^{\prime}\right)\right)$ for all $\zeta \in H^{1}\left(\Omega_{1}^{\prime} \cup \Omega_{2}^{\prime}\right)$, we have

$$
I_{\lambda}^{\prime}(u) \zeta=\Phi_{\lambda}^{\prime}\left(P u+\varphi_{\lambda}(u)\right)(P \zeta) \quad \text { for all } \zeta \in H^{1}\left(\Omega_{1}^{\prime} \cup \Omega_{2}^{\prime}\right) .
$$

From $C^{1}$-continuity of $\varphi_{\lambda}(u)$ and (2.12), it follows that $I_{\lambda}(u)$ is of class $C^{2}$.

Next we observe (iv). By (2.12) the "if" part clearly holds. Suppose that $I_{\lambda}^{\prime}(u)=$ 0 . Thus by (2.11), (2.12) we have

$$
\Phi_{\lambda}^{\prime}\left(w_{\lambda}(u)\right)(P \zeta+h)=0 \quad \text { for all } \zeta \in H^{1}\left(\Omega_{1}^{\prime} \cup \Omega_{2}^{\prime}\right) \text { and } h \in H_{0}^{1}\left(\mathbf{R}^{N} \backslash\left(\Omega_{1}^{\prime} \cup \Omega_{2}^{\prime}\right)\right) \text {. }
$$

Since $H^{1}\left(\mathbf{R}^{N}\right)=\left\{P \zeta+h ; \zeta \in H^{1}\left(\Omega_{1}^{\prime} \cup \Omega_{2}^{\prime}\right), h \in H_{0}^{1}\left(\mathbf{R}^{N} \backslash\left(\Omega_{1}^{\prime} \cup \Omega_{2}^{\prime}\right)\right)\right\}$, we have $\Phi_{\lambda}^{\prime}\left(w_{\lambda}(u)\right)=0$. Thus the "only if" part also holds. 
For (v), we note that

$$
\begin{aligned}
& \left\|\left.\varphi\right|_{\Omega_{1}^{\prime} \cup \Omega_{2}^{\prime}}\right\|_{\lambda, \Omega_{1}^{\prime} \cup \Omega_{2}^{\prime}} \leq\|\varphi\|_{\lambda, \mathbf{R}^{N}}, \\
& \varphi-\left.P \varphi\right|_{\Omega_{1}^{\prime} \cup \Omega_{2}^{\prime}} \in H_{0}^{1}\left(\mathbf{R}^{N} \backslash\left(\Omega_{1}^{\prime} \cup \Omega_{2}^{\prime}\right)\right)
\end{aligned}
$$

for all $\varphi \in H^{1}\left(\mathbf{R}^{N}\right)$. Thus by (2.11), (2.12)

$$
\begin{aligned}
\Phi_{\lambda}^{\prime}\left(w_{\lambda}(u)\right) \varphi & =\Phi_{\lambda}^{\prime}\left(w_{\lambda}(u)\right)\left(\left.P \varphi\right|_{\Omega_{1}^{\prime} \cup \Omega_{2}^{\prime}}+\left(\varphi-\left.P \varphi\right|_{\Omega_{1}^{\prime} \cup \Omega_{2}^{\prime}}\right)\right) \\
& =\Phi_{\lambda}^{\prime}\left(w_{\lambda}(u)\right)\left(\left.P \varphi\right|_{\Omega_{1}^{\prime} \cup \Omega_{2}^{\prime}}\right)=I_{\lambda}^{\prime}(u)\left(\left.\varphi\right|_{\Omega_{1}^{\prime} \cup \Omega_{2}^{\prime}} .\right.
\end{aligned}
$$

Thus for any $\varphi \in H^{1}\left(\mathbf{R}^{N}\right)$ with $\|\varphi\|_{\lambda, \mathbf{R}^{N}} \leq 1$

$$
\left|\Phi_{\lambda}^{\prime}\left(w_{\lambda}(u)\right) \varphi\right| \leq\left\|I_{\lambda}^{\prime}(u)\right\|_{\lambda, \Omega_{1}^{\prime} \cup \Omega_{2}^{\prime}}^{*}\left\|\left.\varphi\right|_{\Omega_{1}^{\prime} \cup \Omega_{2}^{\prime}}\right\|_{\lambda, \Omega_{1}^{\prime} \cup \Omega_{2}^{\prime}} \leq\left\|I_{\lambda}^{\prime}(u)\right\|_{\lambda, \Omega_{1}^{\prime} \cup \Omega_{2}^{\prime}}^{*} .
$$

That is, we have

$$
\left\|\Phi_{\lambda}^{\prime}\left(w_{\lambda}(u)\right)\right\|_{\lambda, \mathbf{R}^{N}}^{*} \leq\left\|I_{\lambda}^{\prime}(u)\right\|_{\lambda, \Omega_{1}^{\prime} \cup \Omega_{2}^{\prime}}^{*}
$$

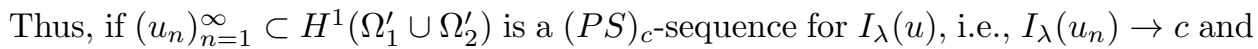
$\left\|I_{\lambda}^{\prime}\left(u_{n}\right)\right\|_{\lambda, \Omega_{1}^{\prime} \cup \Omega_{2}^{\prime}}^{*} \rightarrow 0$, then $\left(w_{\lambda}\left(u_{n}\right)\right)_{n=1}^{\infty} \subset H^{1}\left(\mathbf{R}^{N}\right)$ is a $(P S)_{c^{-}}$sequence for $\Phi_{\lambda}(u)$. By Proposition 2.2, we can see $\left(w_{\lambda}\left(u_{n}\right)\right)_{n=1}^{\infty}$ has a strongly convergent subsequence in $H^{1}\left(\mathbf{R}^{N}\right)$. Thus $u_{n}=\left.w_{\lambda}\left(u_{n}\right)\right|_{\Omega_{1}^{\prime} \cup \Omega_{2}^{\prime}}$ has a strongly convergent subsequence in $H^{1}\left(\Omega_{1}^{\prime} \cup \Omega_{2}^{\prime}\right)$ and $(P S)_{c}$ condition holds for $I_{\lambda}(u)$.

For later use, we consider the following linear equation: let

$$
V(x) \in L^{\infty}\left(\mathbf{R}^{N} \backslash\left(\Omega_{1}^{\prime} \cup \Omega_{2}^{\prime}\right)\right),
$$

and we consider the following linear boundary value problem:

$$
\begin{aligned}
& -\Delta w+\left(\lambda^{2} a(x)+1\right) w=V(x) w \quad \text { in } \mathbf{R}^{N} \backslash\left(\Omega_{1}^{\prime} \cup \Omega_{2}^{\prime}\right), \\
& w=u \quad \text { on } \partial \Omega_{1}^{\prime} \cup \partial \Omega_{2}^{\prime} .
\end{aligned}
$$

We have the following

Lemma 2.6. Suppose that $V(x) \in L^{\infty}\left(\mathbf{R}^{N} \backslash\left(\Omega_{1}^{\prime} \cup \Omega_{2}^{\prime}\right)\right)$ satisfies

$$
\|V(x)\|_{L^{\infty}\left(\mathbf{R}^{N} \backslash\left(\Omega_{1}^{\prime} \cup \Omega_{2}^{\prime}\right)\right)} \leq \frac{2}{3} .
$$

Then the linear boundary value problem (2.13)-(2.14) has a unique solution we denote it by $Q_{V, \lambda}(u)$ - for all $u \in H^{1}\left(\Omega_{1}^{\prime} \cup \Omega_{2}^{\prime}\right)$. Moreover $Q_{V, \lambda}$ defines a bounded linear operator $Q_{V, \lambda}: H^{1}\left(\Omega_{1}^{\prime} \cup \Omega_{2}^{\prime}\right) \rightarrow H^{1}\left(\mathbf{R}^{N} \backslash\left(\Omega_{1}^{\prime} \cup \Omega_{2}^{\prime}\right)\right)$, and there exists a constant $C_{2}>0$ independent of $\lambda \geq 1$ such that

$$
\left\|Q_{V, \lambda}(u)\right\|_{\lambda, \mathbf{R}^{N} \backslash\left(\Omega_{1}^{\prime} \cup \Omega_{2}^{\prime}\right)} \leq C_{2}\|u\|_{\lambda,\left(\Omega_{1}^{\prime} \backslash \Omega_{1}^{(1)}\right) \cup\left(\Omega_{2}^{\prime} \backslash \Omega_{2}^{(1)}\right)} \quad \text { for all } u \in H^{1}\left(\Omega_{1}^{\prime} \cup \Omega_{2}^{\prime}\right) .
$$

Proof of Lemma 2.6. Set

$$
\begin{aligned}
F(w)= & \int_{\mathbf{R}^{N} \backslash\left(\Omega_{1}^{\prime} \cup \Omega_{2}^{\prime}\right)}|\nabla w|^{2}+\left(\lambda^{2} a(x)+1\right) w^{2}-V(x) w^{2} d x \\
& : H^{1}\left(\mathbf{R}^{N} \backslash\left(\Omega_{1}^{\prime} \cup \Omega_{2}^{\prime}\right)\right) \rightarrow \mathbf{R} .
\end{aligned}
$$

Then solutions of (2.13)-(2.14) can be characterized as critical points of $F(w)$ on $A_{u}=\left\{w \in H^{1}\left(\mathbf{R}^{N}\right) ; w=u\right.$ on $\left.\Omega_{1}^{\prime} \cup \Omega_{2}^{\prime}\right\}$. By assumption (2.15) we have

$$
F(w) \geq\|w\|_{\lambda, \mathbf{R}^{N} \backslash\left(\Omega_{1}^{\prime} \cup \Omega_{2}^{\prime}\right)}^{2}-\frac{2}{3}\|w\|_{L^{2}\left(\mathbf{R}^{N} \backslash\left(\Omega_{1}^{\prime} \cup \Omega_{2}^{\prime}\right)\right)}^{2} \geq \frac{1}{3}\|w\|_{\lambda, \mathbf{R}^{N} \backslash\left(\Omega_{1}^{\prime} \cup \Omega_{2}^{\prime}\right)}^{2} .
$$

Thus $F(w)$ is coercive and convex on $A_{u}$ and we have the existence and the uniqueness of solutions (2.13)-(2.14). 
To show (2.16), we note that $Q_{V, \lambda}(u)$ can be characterized as

$$
F\left(Q_{V, \lambda}(u)\right)=\inf _{w \in A_{u}} F(w) \text {. }
$$

In particular, $F\left(Q_{V, \lambda}(u)\right) \leq F(P u)$, where $P u$ is defined in Lemma 2.5 Thus by (2.17) and (2.9)

$$
\begin{aligned}
& \frac{1}{3}\left\|Q_{V, \lambda}(u)\right\|_{\lambda, \mathbf{R}^{N} \backslash\left(\Omega_{1}^{\prime} \cup \Omega_{2}^{\prime}\right)}^{2} \leq F\left(Q_{V, \lambda}(u)\right) \leq F(P u) \\
\leq & \|P u\|_{\lambda, \mathbf{R}^{N} \backslash\left(\Omega_{1}^{\prime} \cup \Omega_{2}^{\prime}\right)}^{2}+\left|\int_{\mathbf{R}^{N} \backslash\left(\Omega_{1}^{\prime} \cup \Omega_{2}^{\prime}\right)} V(x)(P u)^{2} d x\right| \\
\leq & \left(1+\frac{2}{3}\right)\|P u\|_{\lambda, \mathbf{R}^{N} \backslash\left(\Omega_{1}^{\prime} \cup \Omega_{2}^{\prime}\right)}^{2} \\
\leq & \frac{5}{3} C_{1}^{2}\|u\|_{\lambda,\left(\Omega_{1}^{\prime} \backslash \Omega_{1}^{(1)}\right) \cup\left(\Omega_{2}^{\prime} \backslash \Omega_{2}^{(1)}\right)^{2}}^{2}
\end{aligned}
$$

Thus we get (2.16).

Remark 2.7. Since $Q_{V, \lambda}(u)$ is a unique solution of (2.13) -(2.14), we can give another characterization: for $\zeta \in H^{1}\left(\mathbf{R}^{N} \backslash\left(\Omega_{1}^{\prime} \cup \Omega_{2}^{\prime}\right)\right), \zeta=Q_{V, \lambda}(u)$ holds if and only if

$$
\zeta-P u \in H_{0}^{1}\left(\mathbf{R}^{N} \backslash\left(\Omega_{1}^{\prime} \cup \Omega_{2}^{\prime}\right)\right)
$$

and

$$
F^{\prime}(\zeta) \varphi=0 \quad \text { for all } \varphi \in H_{0}^{1}\left(\mathbf{R}^{N} \backslash\left(\Omega_{1}^{\prime} \cup \Omega_{2}^{\prime}\right)\right) .
$$

We remark that $F^{\prime}(\zeta) \varphi=0$ can be rewritten as

$\langle\zeta, \varphi\rangle_{\lambda, \mathbf{R}^{N} \backslash\left(\Omega_{1}^{\prime} \cup \Omega_{2}^{\prime}\right)}-\int_{\mathbf{R}^{N} \backslash\left(\Omega_{1}^{\prime} \cup \Omega_{2}^{\prime}\right)} V(x) \zeta \varphi d x=0 \quad$ for all $\varphi \in H_{0}^{1}\left(\mathbf{R}^{N} \backslash\left(\Omega_{1}^{\prime} \cup \Omega_{2}^{\prime}\right)\right)$.

Remark 2.8. (i) For any $u \in H^{1}\left(\Omega_{1}^{\prime} \cup \Omega_{2}^{\prime}\right)$ we remark that $V(x)=f\left(w_{\lambda}(u)\right) / w_{\lambda}(u)$ satisfies $\|V(x)\|_{L^{\infty}\left(\mathbf{R}^{N} \backslash\left(\Omega_{1}^{\prime} \cup \Omega_{2}^{\prime}\right)\right)} \leq \frac{2}{3}$, that is, $V(x)$ satisfies (2.15). In particular, $w_{\lambda}(x)$ satisfies $w_{\lambda}(u)=Q_{f\left(w_{\lambda}(u)\right) / w_{\lambda}(u), \lambda}(u)$.

(ii) For $h \in H^{1}\left(\Omega_{1}^{\prime} \cup \Omega_{2}^{\prime}\right)$, it holds that

$$
\left(w_{\lambda}^{\prime}(u) h\right)(x)= \begin{cases}h(x) & \text { for } x \in \Omega_{1}^{\prime} \cup \Omega_{2}^{\prime}, \\ \left(Q_{f^{\prime}\left(w_{\lambda}(u)\right), \lambda}(h)\right)(x) & \text { for } x \in \mathbf{R}^{N} \backslash\left(\Omega_{1}^{\prime} \cup \Omega_{2}^{\prime}\right) .\end{cases}
$$

In fact, $w_{\lambda}(u)$ satisfies

$$
\Phi_{\lambda}^{\prime}\left(w_{\lambda}(u)\right) \varphi=0 \quad \text { for all } \varphi \in H_{0}^{1}\left(\mathbf{R}^{N} \backslash\left(\Omega_{1}^{\prime} \cup \Omega_{2}^{\prime}\right)\right) .
$$

Differentiating with respect to $u$, we have $\Phi_{\lambda}^{\prime \prime}\left(w_{\lambda}(u)\right)\left(w_{\lambda}^{\prime}(u) h, \varphi\right)=0 \quad$ for all $h \in H^{1}\left(\Omega_{1}^{\prime} \cup \Omega_{2}^{\prime}\right)$ and $\varphi \in H_{0}^{1}\left(\mathbf{R}^{N} \backslash\left(\Omega_{1}^{\prime} \cup \Omega_{2}^{\prime}\right)\right)$.

That is,

$$
\left\langle w_{\lambda}^{\prime}(u) h, \varphi\right\rangle_{\lambda, \mathbf{R}^{N} \backslash\left(\Omega_{1}^{\prime} \cup \Omega_{2}^{\prime}\right)}-\int_{\mathbf{R}^{N} \backslash\left(\Omega_{1}^{\prime} \cup \Omega_{2}^{\prime}\right)} f^{\prime}\left(w_{\lambda}(u)\right)\left(w_{\lambda}^{\prime}(u) h\right) \varphi d x=0 .
$$

Since $w_{\lambda}(u)-P u \in H_{0}^{1}\left(\mathbf{R}^{N} \backslash\left(\Omega_{1}^{\prime} \cup \Omega_{2}^{\prime}\right)\right)$, we also have

$$
w_{\lambda}^{\prime}(u) h-P h \in H_{0}^{1}\left(\mathbf{R}^{N} \backslash\left(\Omega_{1}^{\prime} \cup \Omega_{2}^{\prime}\right)\right) .
$$

By Remark 2.7 it follows from (2.21) -(2.22) that

$$
\left.\left(w_{\lambda}^{\prime}(u) h\right)\right|_{\mathbf{R}^{N} \backslash\left(\Omega_{1}^{\prime} \cup \Omega_{2}^{\prime}\right)}=Q_{f^{\prime}\left(w_{\lambda}(u)\right), \lambda}(h) .
$$

Since $\left.w_{\lambda}(u)\right|_{\Omega_{1}^{\prime} \cup \Omega_{2}^{\prime}}=u$, we have (2.19). 
In what follows, we identify $H^{1}\left(\Omega_{1}^{\prime} \cup \Omega_{2}^{\prime}\right)$ and $H^{1}\left(\Omega_{1}^{\prime}\right) \oplus H^{1}\left(\Omega_{2}^{\prime}\right)$, and for functions $u_{1} \in H^{1}\left(\Omega_{1}^{\prime}\right), u_{2} \in H^{1}\left(\Omega_{2}^{\prime}\right)$, we write $u=\left(u_{1}, u_{2}\right)$ if $u \in H^{1}\left(\Omega_{1}^{\prime} \cup \Omega_{2}^{\prime}\right)$ satisfies $u_{1}=\left.u\right|_{\Omega_{1}^{\prime}}, u_{2}=\left.u\right|_{\Omega_{2}^{\prime}}$. When $u=\left(u_{1}, u_{2}\right)$, we also write

$$
\begin{aligned}
& I_{\lambda}\left(u_{1}, u_{2}\right) \quad \text { for } \quad I_{\lambda}(u), \\
& Q_{V, \lambda}\left(u_{1}, u_{2}\right) \quad \text { for } \quad Q_{V, \lambda}(u), \\
& \|u\|_{\lambda, \Omega_{1}^{\prime} \cup \Omega_{2}^{\prime}}^{2}=\|u\|_{\lambda, \Omega_{1}^{\prime}}^{2}+\|u\|_{\lambda, \Omega_{2}^{\prime}}^{2} .
\end{aligned}
$$

By (2.19), it is convenient to extend $Q_{V, \lambda}$ as an operator $H^{1}\left(\Omega_{1}^{\prime} \cup \Omega_{2}^{\prime}\right) \rightarrow H^{1}\left(\mathbf{R}^{N}\right)$ by

$$
\left(Q_{V, \lambda} u\right)(x)=u(x) \quad \text { for } x \in \Omega_{1}^{\prime} \cup \Omega_{2}^{\prime} .
$$

We also denote the extended operator by $Q_{V, \lambda}$. With this notation we have

$$
w_{\lambda}^{\prime}(u) h=Q_{f^{\prime}\left(w_{\lambda}(u)\right), \lambda}(h) .
$$

By (iii) of Proposition 2.4, we have for $h \in H^{1}\left(\Omega_{1}^{\prime} \cup \Omega_{2}^{\prime}\right)$

$$
I_{\lambda}^{\prime}(u) h=\Phi_{\lambda}^{\prime}\left(w_{\lambda}(u)\right)\left(w_{\lambda}^{\prime}(u) h\right)=\Phi_{\lambda}^{\prime}\left(w_{\lambda}(u)\right) Q_{f^{\prime}\left(w_{\lambda}(u)\right), \lambda}(h) .
$$

Since $Q_{f^{\prime}\left(w_{\lambda}(u)\right), \lambda}(h)-Q_{0, \lambda}(h) \in H_{0}^{1}\left(\mathbf{R}^{N} \backslash\left(\Omega_{1}^{\prime} \cup \Omega_{2}^{\prime}\right)\right)$, it follows from (2.20) that

$$
I_{\lambda}^{\prime}(u) h=\Phi_{\lambda}^{\prime}\left(w_{\lambda}(u)\right) Q_{0, \lambda}(h) \quad \text { for all } h \in H^{1}\left(\Omega_{1}^{\prime} \cup \Omega_{2}^{\prime}\right) .
$$

Proof of the following estimates will be given in Section 7 .

Lemma 2.9. Let $V(x): \mathbf{R}^{N} \backslash\left(\Omega_{1}^{\prime} \cup \Omega_{2}^{\prime}\right) \rightarrow \mathbf{R}$ be a function satisfying (2.15). Then there exist $C_{3}>0$ independent of $\lambda \geq 1$ and $r_{\lambda}>0$ satisfying $r_{\lambda} \rightarrow 0$ as $\lambda \rightarrow \infty$ such that

(i) $\left\|Q_{V, \lambda}(u)\right\|_{\lambda, \mathbf{R}^{N} \backslash\left(\Omega_{1}^{\prime} \cup \Omega_{2}^{\prime}\right)} \leq C_{3}\|u\|_{\lambda, \Omega_{1}^{\prime} \cup \Omega_{2}^{\prime}}$ for all $u \in H^{1}\left(\Omega_{1}^{\prime} \cup \Omega_{2}^{\prime}\right)$.

(ii) $\left\|Q_{V, \lambda}(u)-Q_{0, \lambda}(u)\right\|_{\lambda, \mathbf{R}^{N} \backslash\left(\Omega_{1}^{\prime} \cup \Omega_{2}^{\prime}\right)} \leq r_{\lambda}\|u\|_{\lambda, \Omega_{1}^{\prime} \cup \Omega_{2}^{\prime}}$ for all $u \in H^{1}\left(\Omega_{1}^{\prime} \cup \Omega_{2}^{\prime}\right)$.

(iii) $\left|\left\langle Q_{0, \lambda}\left(u_{1}, 0\right), Q_{0, \lambda}\left(0, u_{2}\right)\right\rangle_{\lambda, \mathbf{R}^{N} \backslash\left(\Omega_{1}^{\prime} \cup \Omega_{2}^{\prime}\right)}\right| \leq r_{\lambda}\left\|u_{1}\right\|_{\lambda, \Omega_{1}^{\prime}}\left\|u_{2}\right\|_{\lambda, \Omega_{2}^{\prime}}$ for all $u_{1} \in$ $H^{1}\left(\Omega_{1}^{\prime}\right)$ and $u_{2} \in H^{1}\left(\Omega_{2}^{\prime}\right)$.

(iv) $\left\|w_{\lambda}(u)\right\|_{\lambda, \mathbf{R}^{N} \backslash\left(\Omega_{1}^{\prime} \cup \Omega_{2}^{\prime}\right)} \leq C_{3}\|u\|_{\lambda, \Omega_{1}^{\prime} \cup \Omega_{2}^{\prime}}$, $\left\|w_{\lambda}(u)-Q_{0, \lambda}(u)\right\|_{\lambda, \mathbf{R}^{N} \backslash\left(\Omega_{1}^{\prime} \cup \Omega_{2}^{\prime}\right)} \leq r_{\lambda}\|u\|_{\lambda, \Omega_{1}^{\prime} \cup \Omega_{2}^{\prime}}$ for all $u \in H^{1}\left(\Omega_{1}^{\prime} \cup \Omega_{2}^{\prime}\right)$.

Here and throughout this paper we denote by $r_{\lambda}$ various constants independent of $u$ but which depend on $\lambda$ and satisfy $r_{\lambda} \rightarrow 0$ as $\lambda \rightarrow \infty$.

The following functionals correspond to "unperturbed" problems and will be useful later:

$$
\begin{aligned}
I_{1, \lambda}\left(u_{1}\right)= & \frac{1}{2}\left\|u_{1}\right\|_{\lambda, \Omega_{1}^{\prime}}^{2}-\frac{1}{p+1}\left\|u_{1}\right\|_{L^{p+1}\left(\Omega_{1}^{\prime}\right)}^{p+1} \\
& +\frac{1}{2}\left\|Q_{0, \lambda}\left(u_{1}, 0\right)\right\|_{\lambda, \mathbf{R}^{N} \backslash\left(\Omega_{1}^{\prime} \cup \Omega_{2}^{\prime}\right)}^{2} \in C^{2}\left(H^{1}\left(\Omega_{1}^{\prime}\right), \mathbf{R}\right), \\
I_{2, \lambda}\left(u_{2}\right)= & \frac{1}{2}\left\|u_{2}\right\|_{\lambda, \Omega_{2}^{\prime}}^{2}-\frac{1}{p+1}\left\|u_{2}\right\|_{L^{p+1}\left(\Omega_{2}^{\prime}\right)}^{p+1} \\
& +\frac{1}{2}\left\|Q_{0, \lambda}\left(0, u_{2}\right)\right\|_{\lambda, \mathbf{R}^{N} \backslash\left(\Omega_{1}^{\prime} \cup \Omega_{2}^{\prime}\right)}^{2} \in C^{2}\left(H^{1}\left(\Omega_{2}^{\prime}\right), \mathbf{R}\right) .
\end{aligned}
$$

We will study $I_{\lambda}\left(u_{1}, u_{2}\right)$ as a perturbation from $I_{1, \lambda}\left(u_{1}\right)+I_{2, \lambda}\left(u_{2}\right)$.

Lemma 2.10. There exists $r_{\lambda}>0$ such that for $\left(u_{1}, u_{2}\right) \in H^{1}\left(\Omega_{1}^{\prime} \cup \Omega_{2}^{\prime}\right)=$ $H^{1}\left(\Omega_{1}^{\prime}\right) \oplus H^{1}\left(\Omega_{2}^{\prime}\right)$,

(0) $r_{\lambda} \rightarrow 0$ as $\lambda \rightarrow \infty$.

(i) $\left|I_{\lambda}\left(u_{1}, u_{2}\right)-I_{1, \lambda}\left(u_{1}\right)-I_{2, \lambda}\left(u_{2}\right)\right| \leq \frac{1}{2} r_{\lambda}\left(\left\|u_{1}\right\|_{\lambda, \Omega_{1}^{\prime}}+\left\|u_{2}\right\|_{\lambda, \Omega_{2}^{\prime}}\right)^{2}$. 
(ii) $\left\|\frac{\partial I_{\lambda}}{\partial u_{1}}\left(u_{1}, u_{2}\right)-I_{1, \lambda}^{\prime}\left(u_{1}\right)\right\|_{\lambda, \Omega_{1}^{\prime}}^{*} \leq r_{\lambda}\left(\left\|u_{1}\right\|_{\lambda, \Omega_{1}^{\prime}}+\left\|u_{2}\right\|_{\lambda, \Omega_{2}^{\prime}}\right)$.

(iii) $\left\|\frac{\partial I_{\lambda}}{\partial u_{2}}\left(u_{1}, u_{2}\right)-I_{2, \lambda}^{\prime}\left(u_{2}\right)\right\|_{\lambda, \Omega_{2}^{\prime}}^{*} \leq r_{\lambda}\left(\left\|u_{1}\right\|_{\lambda, \Omega_{1}^{\prime}}+\left\|u_{2}\right\|_{\lambda, \Omega_{2}^{\prime}}\right)$.

We use the notation:

$$
\begin{array}{lll}
\left\|u_{1}\right\|_{\lambda, \Omega_{1}^{\prime}, \#}^{2}=\left\|u_{1}\right\|_{\lambda, \Omega_{1}^{\prime}}^{2}+\left\|Q_{0, \lambda}\left(u_{1}, 0\right)\right\|_{\lambda, \mathbf{R}^{N} \backslash\left(\Omega_{1}^{\prime} \cup \Omega_{2}^{\prime}\right)}^{2} & \text { for } u_{1} \in H^{1}\left(\Omega_{1}^{\prime}\right), \\
\left\|u_{2}\right\|_{\lambda, \Omega_{2}^{\prime}, \#}^{2}=\left\|u_{2}\right\|_{\lambda, \Omega_{2}^{\prime}}^{2}+\left\|Q_{0, \lambda}\left(0, u_{2}\right)\right\|_{\lambda, \mathbf{R}^{N} \backslash\left(\Omega_{1}^{\prime} \cup \Omega_{2}^{\prime}\right)}^{2} & \text { for } u_{2} \in H^{1}\left(\Omega_{2}^{\prime}\right) .
\end{array}
$$

Then $\|\cdot\|_{\lambda, \Omega_{i}^{\prime}, \#}$ is a norm on $H^{1}\left(\Omega_{i}^{\prime}\right)$, and we have from Lemma 2.9 that

$$
\|u\|_{\lambda, \Omega_{i}^{\prime}} \leq\|u\|_{\lambda, \Omega_{i}^{\prime}, \#} \leq\left(1+C_{3}\right)\|u\|_{\lambda, \Omega_{i}^{\prime}} \text { for all } u \in H^{1}\left(\Omega_{i}^{\prime}\right)
$$

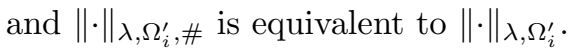

As a corollary to Lemma 2.10, we have

Corollary 2.11. For all $u=\left(u_{1}, u_{2}\right) \in H^{1}\left(\Omega_{1}^{\prime}\right) \oplus H^{1}\left(\Omega_{2}^{\prime}\right)$

$$
\begin{aligned}
\frac{1}{2}(1- & \left.r_{\lambda}\right)\left(\left\|u_{1}\right\|_{\lambda, \Omega_{1}^{\prime}, \#}^{2}+\left\|u_{2}\right\|_{\lambda, \Omega_{2}^{\prime}, \#}^{2}\right)-\frac{1}{p+1}\left(\left\|u_{1}\right\|_{L^{p+1}\left(\Omega_{1}^{\prime}\right)}^{p+1}+\left\|u_{2}\right\|_{L^{p+1}\left(\Omega_{2}^{\prime}\right)}^{p+1}\right) \\
& \leq I_{\lambda}\left(u_{1}, u_{2}\right) \\
& \leq \frac{1}{2}\left(1+r_{\lambda}\right)\left(\left\|u_{1}\right\|_{\lambda, \Omega_{1}^{\prime}, \#}^{2}+\left\|u_{2}\right\|_{\lambda, \Omega_{2}^{\prime}, \#}^{2}\right)-\frac{1}{p+1}\left(\left\|u_{1}\right\|_{L^{p+1}\left(\Omega_{1}^{\prime}\right)}^{p+1}+\left\|u_{2}\right\|_{L^{p+1}\left(\Omega_{2}^{\prime}\right)}^{p+1}\right)
\end{aligned}
$$

$$
\begin{aligned}
& \left(1-r_{\lambda}\right)\left\|u_{1}\right\|_{\lambda, \Omega_{1}^{\prime}, \#}^{2}-\left\|u_{1}\right\|_{L^{p+1}\left(\Omega_{1}^{\prime}\right)}^{p+1}+\left(1-r_{\lambda}\right)\left\|u_{2}\right\|_{\lambda, \Omega_{2}^{\prime}, \#}^{2}-\left\|u_{2}\right\|_{L^{p+1}\left(\Omega_{2}^{\prime}\right)}^{p+1} \\
\leq & I_{\lambda}^{\prime}\left(u_{1}, u_{2}\right)\left(u_{1}, u_{2}\right) \\
\leq & \left(1+r_{\lambda}\right)\left\|u_{1}\right\|_{\lambda, \Omega_{1}^{\prime}, \#}^{2}-\left\|u_{1}\right\|_{L^{p+1}\left(\Omega_{1}^{\prime}\right)}^{p+1}+\left(1+r_{\lambda}\right)\left\|u_{2}\right\|_{\lambda, \Omega_{2}^{\prime}, \#}^{2}-\left\|u_{2}\right\|_{L^{p+1}\left(\Omega_{2}^{\prime}\right)}^{p+1} .
\end{aligned}
$$

We also remark that $I_{i, \lambda}(u)(i=1,2)$ has the following properties:

Lemma 2.12. For $i=1,2$ we have

(i) $I_{i, \lambda}\left(u_{i}\right): H^{1}\left(\Omega_{i}^{\prime}\right) \rightarrow \mathbf{R}$ has a mountain pass geometry. That is, there are constants $r_{0}, \delta_{0}>0$ and $e_{i} \in H_{0}^{1}\left(\Omega_{i}^{\prime}\right)$ independent of $\lambda \geq 1$ such that

$$
\begin{aligned}
& I_{i, \lambda}\left(u_{i}\right) \geq \delta_{0} \quad \text { for all } u_{i} \in H^{1}\left(\Omega_{i}^{\prime}\right) \text { with }\left\|u_{i}\right\|_{\lambda, \Omega_{i}^{\prime}}=r_{0}, \\
& \left\|e_{i}\right\|_{\lambda, \Omega_{i}^{\prime}}>r_{0} \quad \text { and } I_{i, \lambda}\left(e_{i}\right)<0 .
\end{aligned}
$$

(ii) $I_{i, \lambda}\left(u_{i}\right)$ satisfies the $(P S)_{c}$-condition for all $c \in \mathbf{R}$.

Proof of Lemma 2.12. For $i=1,2$, we remark that $I_{i, \lambda}(u)$ can be written as

$$
I_{i, \lambda}(u)=\frac{1}{2}\|u\|_{\lambda, \Omega_{i}^{\prime}, \#}^{2}-\frac{1}{p+1}\|u\|_{L^{p+1}\left(\Omega_{i}^{\prime}\right)}^{p+1},
$$

and we can check (i), (ii) in a rather standard way.

(d) Reduction to a problem on $\Sigma_{1, \lambda} \times \Sigma_{2, \lambda}$. Next we reduce our problem on an infinite dimensional torus $\Sigma_{1, \lambda} \times \Sigma_{2, \lambda}$, where

$$
\Sigma_{i, \lambda}=\left\{v \in H^{1}\left(\Omega_{i}^{\prime}\right) ;\|v\|_{\lambda, \Omega_{i}^{\prime}}=1\right\} \quad \text { for } i=1,2 .
$$

We define $J_{i, \lambda}: \Sigma_{i, \lambda} \rightarrow(0, \infty](i=1,2), J_{\lambda}: \Sigma_{1, \lambda} \times \Sigma_{2, \lambda} \rightarrow(0, \infty]$ by

$$
J_{i, \lambda}\left(v_{i}\right)=\sup _{s \geq 0} I_{i, \lambda}\left(s v_{i}\right), \quad J_{\lambda}\left(v_{1}, v_{2}\right)=\sup _{s, t \geq 0} I_{\lambda}\left(s v_{1}, t v_{2}\right) .
$$


For $J_{i, \lambda}\left(v_{i}\right)(i=1,2)$, we can easily see that $I_{i, \lambda}\left(s v_{i}\right):[0, \infty) \rightarrow \mathbf{R}$ takes a global maximum at

$$
s=s_{i, \lambda}\left(v_{i}\right)=\left\|v_{i}\right\|_{\lambda, \Omega_{i}^{\prime}, \#}^{\frac{2}{p-1}}\left\|v_{i}\right\|_{L^{p+1}\left(\Omega_{i}^{\prime}\right)}^{-\frac{p+1}{p-1}}
$$

and

$$
J_{i, \lambda}\left(v_{i}\right)=I_{i, \lambda}\left(s_{i, \lambda}\left(v_{i}\right) v_{i}\right)=\left(\frac{1}{2}-\frac{1}{p+1}\right)\left(\frac{\left\|v_{i}\right\|_{\lambda, \Omega_{i}^{\prime}, \#}}{\left\|v_{i}\right\|_{L^{p+1}\left(\Omega_{i}^{\prime}\right)}}\right)^{\frac{2(p+1)}{p-1}} .
$$

Recalling $\left\|v_{i}\right\|_{H^{1}\left(\Omega_{i}^{\prime}\right)} \leq\left\|v_{i}\right\|_{\lambda, \Omega_{i}^{\prime}} \leq\left\|v_{i}\right\|_{\lambda, \Omega_{i}^{\prime}, \#}$ for $v_{i} \in \Sigma_{i, \lambda}$, we also have

$$
\begin{aligned}
\inf _{v_{i} \in \Sigma_{i, \lambda}} J_{i, \lambda}\left(v_{i}\right) & =\left(\frac{1}{2}-\frac{1}{p+1}\right)\left(\inf _{v_{i} \in H^{1}\left(\Omega_{i}^{\prime}\right), v_{i} \neq 0} \frac{\left\|v_{i}\right\|_{\lambda, \Omega_{i}^{\prime}, \#}}{\left\|v_{i}\right\|_{L^{p+1}\left(\Omega_{i}^{\prime}\right)}}\right)^{\frac{2(p+1)}{p-1}} \\
& \geq m_{i} \equiv\left(\frac{1}{2}-\frac{1}{p+1}\right)\left(\inf _{v_{i} \in H^{1}\left(\Omega_{i}^{\prime}\right), v_{i} \neq 0} \frac{\left\|v_{i}\right\|_{H^{1}\left(\Omega_{i}^{\prime}\right)}}{\left\|v_{i}\right\|_{L^{p+1}\left(\Omega_{i}^{\prime}\right)}}\right)^{\frac{2(p+1)}{p-1}} .
\end{aligned}
$$

Remark 2.13. In a similar way to (2.28) we have for $J_{i, D}\left(v_{i}\right)$ defined in (1.7)

$$
J_{i, D}\left(v_{i}\right)=\left(\frac{1}{2}-\frac{1}{p+1}\right)\left(\frac{1}{\left\|v_{i}\right\|_{L^{p+1}\left(\Omega_{i}\right)}}\right)^{\frac{2(p+1)}{p-1}} \quad \text { for } v_{i} \in \Sigma_{i, D}
$$

For $J_{\lambda}\left(v_{1}, v_{2}\right)$ we have

Lemma 2.14. $\quad$ (i) There exists $r_{\lambda}>0$ satisfying $r_{\lambda} \rightarrow 0$ as $\lambda \rightarrow \infty$ such that $\left(1-r_{\lambda}\right)^{\frac{2(p+1)}{p-1}}\left(J_{1, \lambda}\left(v_{1}\right)+J_{2, \lambda}\left(v_{2}\right)\right) \leq J_{\lambda}\left(v_{1}, v_{2}\right) \leq\left(1+r_{\lambda}\right)^{\frac{2(p+1)}{p-1}}\left(J_{1, \lambda}\left(v_{1}\right)+J_{2, \lambda}\left(v_{2}\right)\right)$ for all $\left(v_{1}, v_{2}\right) \in \Sigma_{1, \lambda} \times \Sigma_{2, \lambda}$.

(ii) $\inf _{\left(v_{1}, v_{2}\right) \in \Sigma_{1, \lambda} \times \Sigma_{2, \lambda}} J_{\lambda}\left(v_{1}, v_{2}\right) \geq m_{1}+m_{2}$, where $m_{1}, m_{2}>0$ are defined in (2.29).

Proof. (i) easily follows from (i) of Corollary 2.11. For (ii), since $\left\|u_{i}\right\|_{\lambda, \Omega_{i}^{\prime}} \geq$ $\left\|u_{i}\right\|_{H^{1}\left(\Omega_{i}^{\prime}\right)}$ we have

$I_{\lambda}\left(u_{1}, u_{2}\right) \geq \frac{1}{2}\left(\left\|u_{1}\right\|_{H^{1}\left(\Omega_{1}^{\prime}\right)}^{2}+\left\|u_{2}\right\|_{H^{1}\left(\Omega_{2}^{\prime}\right)}^{2}\right)-\frac{1}{p+1}\left(\left\|u_{1}\right\|_{L^{p+1}\left(\Omega_{1}^{\prime}\right)}^{p+1}+\left\|u_{2}\right\|_{L^{p+1}\left(\Omega_{2}^{\prime}\right)}^{p+1}\right)$.

Thus (ii) follows.

In what follows, we use the following notation: for $M \in \mathbf{R}$

$$
\begin{aligned}
& {\left[J_{\lambda} \leq M\right]_{\Sigma_{1, \lambda} \times \Sigma_{2, \lambda}}=\left\{\left(v_{1}, v_{2}\right) \in \Sigma_{1, \lambda} \times \Sigma_{2, \lambda} ; J_{\lambda}\left(v_{1}, v_{2}\right) \leq M\right\},} \\
& {\left[J_{\lambda}<M\right]_{\Sigma_{1, \lambda} \times \Sigma_{2, \lambda}}=\left\{\left(v_{1}, v_{2}\right) \in \Sigma_{1, \lambda} \times \Sigma_{2, \lambda} ; J_{\lambda}\left(v_{1}, v_{2}\right)<M\right\} .}
\end{aligned}
$$

The following propositions state fundamental properties of $J_{\lambda}\left(v_{1}, v_{2}\right)$, which we will use repeatedly in the rest of the paper.

Proposition 2.15. For any $M>0$ there exist constants $\lambda(M), \delta_{M}>0$ such that for $\lambda \geq \lambda(M)$

(i) $\left\|v_{1}\right\|_{L^{p+1}\left(\Omega_{1}^{\prime}\right)}^{p+1} \geq \delta_{M},\left\|v_{2}\right\|_{L^{p+1}\left(\Omega_{2}^{\prime}\right)}^{p+1} \geq \delta_{M}$ for all $\left(v_{1}, v_{2}\right) \in\left[J_{\lambda} \leq M\right]_{\Sigma_{1, \lambda} \times \Sigma_{2, \lambda}}$.

(ii) For any $\left(v_{1}, v_{2}\right) \in\left[J_{\lambda} \leq M\right]_{\Sigma_{1, \lambda} \times \Sigma_{2, \lambda}},(s, t) \mapsto J_{\lambda}\left(s v_{1}, t v_{2}\right)$ has a unique maximizer - we denote $\left(s_{\lambda}\left(v_{1}, v_{2}\right), t_{\lambda}\left(v_{1}, v_{2}\right)\right)$ - and it is nondegenerate. 
(iii) There exist constants $R_{0}, R_{1}(M)>0-R_{1}(M)>0$ depends on $M>0$ but is independent of $\left(v_{1}, v_{2}\right)$, and $R_{0}$ is independent of $M,\left(v_{1}, v_{2}\right)$ - such that

$$
\begin{aligned}
& s_{\lambda}\left(v_{1}, v_{2}\right)^{2}+t_{\lambda}\left(v_{1}, v_{2}\right)^{2} \leq R_{1}(M)^{2}, \\
& s_{\lambda}\left(v_{1}, v_{2}\right), t_{\lambda}\left(v_{1}, v_{2}\right) \geq R_{0} .
\end{aligned}
$$

(iv) $\left(s_{\lambda}\left(v_{1}, v_{2}\right), t_{\lambda}\left(v_{1}, v_{2}\right)\right):\left[J_{\lambda}<M\right]_{\Sigma_{1, \lambda} \times \Sigma_{2, \lambda}} \rightarrow \mathbf{R}^{2}$ is of class $C^{1}$ and $J_{\lambda}$ : $\left[J_{\lambda}<M\right]_{\Sigma_{1, \lambda} \times \Sigma_{2, \lambda}} \rightarrow \mathbf{R}$ is of class $C^{1}$.

(v) There exists $r_{\lambda}>0$ such that

$$
\begin{aligned}
& r_{\lambda} \rightarrow 0 \quad \text { as } \lambda \rightarrow \infty, \\
& \left|J_{\lambda}\left(v_{1}, v_{2}\right)-J_{1, \lambda}\left(v_{1}\right)-J_{2, \lambda}\left(v_{2}\right)\right|<r_{\lambda}, \\
& \quad\left|J_{\lambda}^{\prime}\left(v_{1}, v_{2}\right)\left(h_{1}, h_{2}\right)-J_{1, \lambda}^{\prime}\left(v_{1}\right) h_{1}-J_{2, \lambda}^{\prime}\left(v_{2}\right) h_{2}\right| \\
& \quad \quad<r_{\lambda}\left(\left\|h_{1}\right\|_{\lambda, \Omega_{1}^{\prime}}+\left\|h_{2}\right\|_{\lambda, \Omega_{2}^{\prime}}\right)
\end{aligned}
$$

for all $\left(v_{1}, v_{2}\right) \in\left[J_{\lambda}<M\right]_{\Sigma_{1, \lambda} \times \Sigma_{2, \lambda}}$ and $\left(h_{1}, h_{2}\right) \in H^{1}\left(\Omega_{1}^{\prime}\right) \oplus H^{1}\left(\Omega_{2}^{\prime}\right)$ satisfying $\left\langle v_{1}, h_{1}\right\rangle_{\lambda, \Omega_{1}^{\prime}}=\left\langle v_{2}, h_{2}\right\rangle_{\lambda, \Omega_{2}^{\prime}}=0$.

Proposition 2.16. For $M>0$ suppose $\lambda \geq \lambda(M)$. Then $J_{\lambda}\left(v_{1}, v_{2}\right)$ satisfies the $(P S)_{c}$-condition for $c \in(-\infty, M)$.

Propositions 2.15 and 2.16 enable us to construct deformation flows to ensure the existence of critical points $J_{\lambda}\left(v_{1}, v_{2}\right)$. In Section 9 we will construct deformation flows in various settings.

(e) Convergence and positivity of critical points. The following proposition describes the behavior of critical points $v_{\lambda}=\left(v_{1 \lambda}, v_{2 \lambda}\right) \in \Sigma_{1, \lambda} \times \Sigma_{2, \lambda}$ as $\lambda \rightarrow \infty$.

Proposition 2.17. Suppose that $v_{\lambda}=\left(v_{1 \lambda}, v_{2 \lambda}\right) \in \Sigma_{1, \lambda} \times \Sigma_{2, \lambda}$ satisfies for some $c>0$

$$
\left\|J_{\lambda}^{\prime}\left(v_{\lambda}\right)\right\|_{\left(T_{v_{\lambda}}\left(\Sigma_{1, \lambda} \times \Sigma_{2, \lambda}\right)\right)^{*}} \rightarrow 0, \quad J_{\lambda}\left(v_{\lambda}\right) \rightarrow c \quad \text { as } \lambda \rightarrow \infty .
$$

Then after extracting a subsequence $\lambda_{n} \rightarrow \infty$ we have

(i) There exists $v_{\infty}=\left(v_{1 \infty}, v_{2 \infty}\right) \in \Sigma_{1, D} \times \Sigma_{2, D}$ such that for $i=1,2$

$$
\begin{gathered}
\left\|v_{i \lambda_{n}}-v_{i \infty}\right\|_{H^{1}\left(\Omega_{i}\right)} \rightarrow 0, \quad\left\|v_{i \lambda_{n}}\right\|_{\lambda_{n}, \Omega_{i}^{\prime} \backslash \Omega_{i}} \rightarrow 0 \quad \text { as } n \rightarrow \infty, \\
J_{1, D}^{\prime}\left(v_{1 \infty}\right)=0, \quad J_{2, D}^{\prime}\left(v_{2 \infty}\right)=0, \\
J_{i, D}\left(v_{i \infty}\right)=\lim _{n \rightarrow \infty} J_{i, \lambda_{n}}\left(v_{i \lambda_{n}}\right), \quad J_{1, D}\left(v_{1 \infty}\right)+J_{2, D}\left(v_{2 \infty}\right)=c .
\end{gathered}
$$

(ii) Define $u_{\lambda}=\left(u_{1 \lambda}, u_{2 \lambda}\right) \in H^{1}\left(\Omega_{1}^{\prime} \cup \Omega_{2}^{\prime}\right)=H^{1}\left(\Omega_{1}^{\prime}\right) \oplus H^{1}\left(\Omega_{2}^{\prime}\right)$ and $\tilde{u}_{\lambda} \in$ $H^{1}\left(\mathbf{R}^{N}\right)$ by

$$
u_{\lambda}=\left(u_{1 \lambda}, u_{2 \lambda}\right)=\left(s_{\lambda}\left(v_{\lambda}\right) v_{1 \lambda}, t_{\lambda}\left(v_{\lambda}\right) v_{2 \lambda}\right), \quad \tilde{u}_{\lambda}=w_{\lambda}\left(u_{\lambda}\right) .
$$

Then

$$
\Phi_{\lambda}\left(\tilde{u}_{\lambda}\right)=I_{\lambda}\left(u_{\lambda}\right)=J_{\lambda}\left(v_{\lambda}\right) \rightarrow c, \quad \Phi_{\lambda}^{\prime}\left(\tilde{u}_{\lambda}\right) \rightarrow 0, \quad I_{\lambda}^{\prime}\left(u_{\lambda}\right) \rightarrow 0
$$

as $\lambda \rightarrow \infty$. After extracting a subsequence $\lambda_{n} \rightarrow \infty$, there exists $u_{\infty}=$ $\left(u_{1 \infty}, u_{2 \infty}\right) \in H_{0}^{1}\left(\Omega_{1} \cup \Omega_{2}\right)=H_{0}^{1}\left(\Omega_{1}\right) \oplus H_{0}^{1}\left(\Omega_{2}\right)$ such that

$1^{\circ}\left\|\tilde{u}_{\lambda_{n}}-u_{\infty}\right\|_{\lambda_{n}, \mathbf{R}^{N}} \rightarrow 0$ as $n \rightarrow \infty$.

$2^{\circ} u_{i \infty}$ solves (2.4) -2.5 .

$3^{\circ} \Psi_{1, D}\left(u_{1 \infty}\right)+\Psi_{2, D}\left(u_{2 \infty}\right)=c$.

$4^{\circ} u_{1 \infty} \not \equiv 0$ in $\Omega_{1}, u_{2 \infty} \not \equiv 0$ in $\Omega_{2}$ and $v_{i \infty}=\frac{u_{i \infty}}{\left\|u_{i \infty}\right\|_{H^{1}\left(\Omega_{i}\right)}}$ for $i=1,2$. 
Proof. First we show $1^{\circ}-4^{\circ}$ of the second statement (ii). Suppose that $v_{\lambda}=$ $\left(v_{1 \lambda}, v_{2 \lambda}\right) \in \Sigma_{1, \lambda} \times \Sigma_{2, \lambda}$ satisfies (2.34). Defining $u_{\lambda}, \tilde{u}_{\lambda}$ by (2.38), we can see that there exist $R_{0}, R_{1}>0$ such that

$$
s_{\lambda}\left(v_{\lambda}\right), t_{\lambda}\left(v_{\lambda}\right) \in\left[R_{0}, R_{1}\right] \text { for large } \lambda .
$$

By Proposition 2.3, there exists a subsequence $\lambda_{n} \rightarrow \infty$ and $u_{\infty}=\left(u_{1 \infty}, u_{2 \infty}\right) \in$ $H_{0}^{1}\left(\Omega_{1}\right) \oplus H_{0}^{1}\left(\Omega_{2}\right)$ such that $1^{\circ}-2^{\circ}$ hold. By (2.39), we have $u_{1 \infty} \not \equiv 0, u_{2 \infty} \not \equiv 0$ and $3^{\circ}$.

Setting $v_{i \infty}=u_{i \infty} /\left\|u_{i \infty}\right\|_{H^{1}\left(\Omega_{i}\right)} \in \Sigma_{i, D}$ for $i=1,2$, we can deduce (2.35) (2.37) from (2.39) and (ii).

In a way similar to Proposition 2.17, we have

Lemma 2.18. Let $i \in\{1,2\}$ and $v_{\lambda} \in \Sigma_{i, \lambda}$ satisfies for some $c>0$

$$
\left\|J_{i, \lambda}\left(v_{\lambda}\right)\right\|_{\left(T_{v_{\lambda}}\left(\Sigma_{i, \lambda}\right)\right)^{*}} \rightarrow 0, \quad J_{i, \lambda}\left(v_{\lambda}\right) \rightarrow c \quad \text { as } \lambda \rightarrow \infty .
$$

Then after extracting a subsequence $\lambda_{n} \rightarrow \infty$, there exists $v_{\infty} \in \Sigma_{i, D}$ such that

$$
\begin{gathered}
\left\|v_{\lambda_{n}}-v_{\infty}\right\|_{H^{1}\left(\Omega_{i}\right)} \rightarrow 0, \quad\left\|v_{\lambda_{n}}\right\|_{\lambda_{n}, \Omega_{i}^{\prime} \backslash \Omega_{i}} \rightarrow 0, \\
J_{i, D}^{\prime}\left(v_{\infty}\right)=0, \quad J_{i, D}\left(v_{\infty}\right)=c .
\end{gathered}
$$

To see the positivity of the limit $v_{1 \infty}$ in $\Omega_{1}$ (equivalently $u_{1 \infty}$ ), the following proposition is useful.

Proposition 2.19. For any $M>0$ there exists $\delta_{0}(M)>0$ such that for $i=1,2$

(i) If $v \in \Sigma_{i, D}$ satisfies

$$
J_{i, D}^{\prime}(v)=0, \quad J_{i, D}(v) \leq M, \quad\left\|v_{-}\right\|_{L^{p+1}\left(\Omega_{i}\right)}^{p+1} \leq \delta_{0}(M),
$$

then $v>0$ in $\Omega_{1}$.

(ii) If $v_{\lambda}=\left(v_{1 \lambda}, v_{2 \lambda}\right) \in \Sigma_{1, \lambda} \times \Sigma_{2, \lambda}$ satisfies

$$
J_{\lambda}^{\prime}\left(v_{\lambda}\right)=0, \quad J_{\lambda}\left(v_{\lambda}\right) \leq M, \quad\left\|\left(v_{1 \lambda}\right)_{-}\right\|_{L^{p+1}\left(\Omega_{1}\right)}^{p+1} \leq \delta_{0}(M),
$$

then, after extracting a subsequence $\lambda_{n} \rightarrow \infty$ there exists $v_{\infty}=\left(v_{1 \infty}, v_{2 \infty}\right) \in$ $\Sigma_{1, D} \times \Sigma_{2, D}$ such that the conclusion of Proposition 2.17 holds, and moreover $v_{1 \infty}>0$ in $\Omega_{1}$.

(iii) If $v_{\lambda}=\left(v_{1 \lambda}, v_{2 \lambda}\right) \in \Sigma_{1, \lambda} \times \Sigma_{2, \lambda}$ satisfies

$$
\begin{aligned}
& J_{\lambda}^{\prime}\left(v_{\lambda}\right)=0, \quad J_{\lambda}\left(v_{\lambda}\right) \leq M, \\
& \left\|\left(v_{1 \lambda}\right)_{-}\right\|_{L^{p+1}\left(\Omega_{1}^{\prime}\right)}^{p+1} \leq \delta_{0}(M), \quad\left\|\left(v_{2 \lambda}\right)_{-}\right\|_{L^{p+1}\left(\Omega_{2}^{\prime}\right)}^{p+1} \leq \delta_{0}(M),
\end{aligned}
$$

then there exists $\lambda_{M} \geq 1$ such that for large $\lambda \geq \lambda_{M}, \tilde{u}_{\lambda}>0$ in $\mathbf{R}^{N}$. Here $\tilde{u}_{\lambda}$ is defined in (2.38).

Proof. First we remark that there exists $\delta_{1}>0$ such that for $u \in H_{0}^{1}\left(\Omega_{i}\right)$

$$
-\Delta u+u=|u|^{p-1} u \quad \text { in } \Omega_{i}, \quad\left\|u_{-}\right\|_{L^{p+1}\left(\Omega_{i}\right)} \leq \delta_{1}
$$

implies $u_{-} \equiv 0$. In fact, multiplying $u_{-}(x)$ to (2.42) and integrating over $\Omega_{i}$, we have

$$
\left\|u_{-}\right\|_{H^{1}\left(\Omega_{i}\right)}^{2}=\left\|u_{-}\right\|_{L^{p+1}\left(\Omega_{i}\right)}^{p+1} \leq \delta_{1}^{p-1}\left\|u_{-}\right\|_{L^{p+1}\left(\Omega_{i}\right)}^{2} \leq C \delta_{1}^{p-1}\left\|u_{-}\right\|_{H^{1}\left(\Omega_{i}\right)}^{2} .
$$

Thus, choosing $\delta_{1}>0$ small so that $C \delta_{1}^{p-1}<1$, we have $u_{-} \equiv 0$. 
The statements (i), (ii) easily follow from the above property and Proposition 2.17. In a similar way, (iii) follows from the following fact: there exists $\delta_{2}>0$ independent of $\lambda$ such that for $u \in H^{1}\left(\mathbf{R}^{N}\right)$

$$
-\Delta u+\left(\lambda^{2} a(x)+1\right) u=g(x, u) \quad \text { in } \mathbf{R}^{N}, \quad\left\|u_{-}\right\|_{L^{p+1}\left(\mathbf{R}^{N}\right)} \leq \delta_{2}
$$

implies $u_{-} \equiv 0$.

\section{Sign-ChANGING SOlUtions}

We devote Sections 34 for a proof of Theorem 1.1. We develop a perturbation theory, and we study $J_{\lambda}\left(v_{1}, v_{2}\right)$ as a perturbation from $J_{1, \lambda}\left(v_{1}\right)+J_{2, \lambda}\left(v_{2}\right)$. First we study basic properties of our "unperturbed" functionals $J_{i, \lambda}\left(v_{i}\right)$.

(a) Minimax methods for $J_{i, \lambda}\left(v_{i}\right)$. As we observed in Section 2, $I_{1, \lambda}\left(u_{1}\right)$ and $I_{2, \lambda}\left(u_{2}\right)$ have a mountain pass geometry and we can apply the mountain pass theorem. Moreover $I_{2, \lambda}\left(u_{2}\right)$ possesses $\mathbf{Z}_{2}$-symmetry: $I_{2, \lambda}\left(-u_{2}\right)=I_{2, \lambda}\left(u_{2}\right)$, and it enables us to apply symmetric mountain pass theorems. Here we introduce related minimax methods for $J_{1, \lambda}\left(v_{1}\right)$ and $J_{2, \lambda}\left(v_{2}\right)$.

We define minimax values $c_{\text {min }}^{1, \lambda}, b_{n}^{2, \lambda}(n \in \mathbf{N})$ by

$$
c_{\text {min }}^{1, \lambda}=\inf _{v_{1} \in \Sigma_{1, \lambda}} J_{1, \lambda}\left(v_{1}\right), \quad b_{n}^{2, \lambda}=\inf _{\gamma \in \Gamma_{n}^{2, \lambda}} \max _{\theta \in S^{n}} J_{2, \lambda}(\gamma(\theta)),
$$

where $S^{n}=\left\{\theta=\left(\theta_{1}, \cdots, \theta_{n+1}\right) ;|\theta|=1\right\}$ and

$$
\Gamma_{n}^{2, \lambda}=\left\{\gamma \in C\left(S^{n}, \Sigma_{2, \lambda}\right) ; \gamma(-\theta)=-\gamma(\theta) \text { for all } \theta \in S^{n}\right\} .
$$

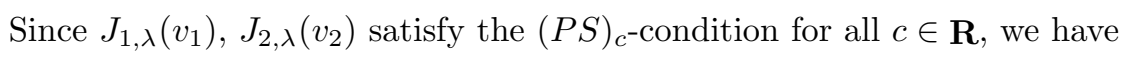

Lemma 3.1. (i) $c_{\text {min }}^{1, \lambda}$ is a critical value of $J_{1, \lambda}\left(v_{1}\right)$.

(ii) $b_{n}^{2, \lambda}$ is a critical value of $J_{2, \lambda}\left(v_{2}\right)$.

(iii) $b_{1}^{2, \lambda} \leq b_{2}^{2, \lambda} \leq \cdots \leq b_{n}^{2, \lambda} \leq b_{n+1}^{2, \lambda} \leq \cdots$.

(iv) $b_{n}^{2, \lambda} \rightarrow \infty$ as $n \rightarrow \infty$.

The main result of this subsection is the following

Proposition 3.2. Let $c_{\min }^{1, D}\left(b_{n}^{2, D}\right.$, respectively) be a critical value of $J_{1, D}\left(v_{1}\right) \in$ $C^{1}\left(\Sigma_{1, D}, \mathbf{R}\right)\left(J_{2, D}\left(v_{2}\right) \in C^{1}\left(\Sigma_{2, D}, \mathbf{R}\right)\right.$, respectively) defined in (1.8) (1.9), respectively). Then we have

(i) $c_{\min }^{1, \lambda} \rightarrow c_{\min }^{1, D}$ as $\lambda \rightarrow \infty$.

(ii) $b_{n}^{2, \lambda} \rightarrow b_{n}^{2, D}$ as $\lambda \rightarrow \infty$.

Proof. We deal with (ii). (i) can be proved in a similar way. We regard $H_{0}^{1}\left(\Omega_{2}\right) \subset$ $H^{1}\left(\Omega_{2}^{\prime}\right)$, and thus we have $\Sigma_{2, D} \subset \Sigma_{2, \lambda}$ and $J_{2, \lambda}(v)=J_{2, D}(v)$ for all $v \in \Sigma_{2, D}$. We also remark that $\Gamma_{n}^{2, D} \subset \Gamma_{n}^{2, \lambda}$. Thus we have

$$
b_{n}^{2, \lambda} \leq b_{n}^{2, D} \quad \text { for all } n \in \mathbf{N} \text { and } \lambda \geq 1 .
$$

We will show that

$$
\liminf _{\lambda \rightarrow \infty} b_{n}^{2, \lambda} \geq b_{n}^{2, D} .
$$

To show (3.2), we will show the following. 
Lemma 3.3. For any $m>0, \delta>0$ and any open set $O \subset \subset \Omega_{2}$, there exists $\lambda_{m, \delta, O} \geq 1$ with the following property: for $\lambda \geq \lambda_{m, \delta, O}$ there exists an odd continuous operator $R_{\lambda}:\left\{v \in \Sigma_{2, \lambda} ; J_{2, \lambda}(v) \leq m\right\} \rightarrow \Sigma_{2, D}$ such that

$$
J_{2, D}\left(R_{\lambda} v\right) \leq(1+\delta) J_{2, \lambda}(v) \quad \text { for all } v \in \Sigma_{2, \lambda} \text { with } J_{2, \lambda}(v) \leq m
$$

and

$$
R_{\lambda} v=v \quad \text { for all } v \in \Sigma_{2, \lambda} \cap H_{0}^{1}(O) \text { with } J_{2, \lambda}(v) \leq m .
$$

We postpone a proof of Lemma 3.3 and we give a proof of Proposition 3.2 .

Proof of Proposition 3.2. We show (3.2). Set $m=b_{n}^{2, D}+1$ and choose $\delta \in(0,1)$ arbitrary. (Property (3.4) is not used here and $O \subset \subset \Omega_{2}$ can be fixed arbitrarily. (3.4) will be used later in the proof of Lemma 3.6.) By Lemma 3.3, there exists an odd continuous mapping $R_{\lambda}$ satisfying (3.3). Choose $\gamma(\theta) \in \Gamma_{n}^{2, \lambda}$ satisfying $\max _{\theta \in S^{n}} J_{2, \lambda}(\gamma(\theta)) \leq b_{n}^{2, \lambda}+\delta$. Recalling (3.1), we have $J_{2, \lambda}(\gamma(\theta)) \leq m$ for all $\theta \in S^{n}$. We set $\tilde{\gamma}(\theta)=R_{\lambda}(\gamma(\theta)) \in \Gamma_{n}^{2, D}$. By (3.3),

$J_{2, D}(\tilde{\gamma}(\theta))=J_{2, D}\left(R_{\lambda}(\gamma(\theta))\right) \leq(1+\delta) J_{2, \lambda}(\gamma(\theta)) \leq(1+\delta)\left(b_{n}^{2, \lambda}+\delta\right) \quad$ for all $\theta \in S^{n}$.

Thus

$$
b_{n}^{2, D} \leq \max _{\theta \in S^{n}} J_{2, D}(\tilde{\gamma}(\theta)) \leq(1+\delta)\left(b_{n}^{2, \lambda}+\delta\right) \quad \text { for } \lambda \geq \lambda_{m, \delta} .
$$

Therefore $b_{n}^{2, D} \leq(1+\delta)\left(\liminf _{\lambda \rightarrow \infty} b_{n}^{2, \lambda}+\delta\right)$. Since $\delta \in(0,1)$ is arbitrary, (3.2) holds.

Next we prove Lemma 3.3 .

Proof of Lemma 3.3. We use the following notation: for an open set $\tilde{\Omega} \subset \mathbf{R}^{N}$ we write

$$
\begin{aligned}
\Sigma_{\tilde{\Omega}} & =\left\{v \in H_{0}^{1}(\tilde{\Omega}) ;\|v\|_{H^{1}(\tilde{\Omega})}=1\right\}, \\
J_{\tilde{\Omega}}(v) & =\left(\frac{1}{2}-\frac{1}{p+1}\right)\left(\frac{1}{\|v\|_{L^{p+1}(\tilde{\Omega})}}\right)^{\frac{2(p+1)}{p-1}} \in C^{1}\left(\Sigma_{\tilde{\Omega}}, \mathbf{R}\right) .
\end{aligned}
$$

Proof of Lemma 3.3 consists of 3 steps.

Step 1: For any $\tilde{\Omega}$ satisfying $\Omega_{2} \subset \subset \tilde{\Omega} \subset \subset \Omega_{2}^{\prime}$ and $m>0, \epsilon>0$, there exists $\lambda_{\tilde{\Omega}, \epsilon, m} \geq 1$ and an odd continuous operator

$$
R_{\lambda}^{1}:\left\{v \in \Sigma_{2, \lambda} ; J_{2, \lambda}(v) \leq m\right\} \rightarrow \Sigma_{\tilde{\Omega}} \quad\left(\lambda \geq \lambda_{\tilde{\Omega}, \epsilon, m}\right),
$$

such that

$$
\begin{aligned}
& J_{\tilde{\Omega}}\left(R_{\lambda}^{1} v\right) \leq(1+\epsilon) J_{2, \lambda}(v) \quad \text { for all } v \in \Sigma_{2, \lambda} \text { with } J_{2, \lambda}(v) \leq m, \\
& R_{\lambda}^{1} v=v \quad \text { for all } v \in \Sigma_{2, \lambda} \cap H_{0}^{1}\left(\Omega_{2}\right) \text { with } J_{2, \lambda}(v) \leq m .
\end{aligned}
$$

Step 2: For any $\epsilon^{\prime}>0$ and any open set $O \subset \subset \Omega_{2}$ there exist a smooth open set $\tilde{\Omega}$ satisfying $\Omega_{2} \subset \subset \tilde{\Omega} \subset \subset \Omega_{2}^{\prime}$ and an odd continuous operator $R^{2}: \Sigma_{\tilde{\Omega}} \rightarrow$ $\Sigma_{2, D}$ such that

$$
\begin{aligned}
& J_{2, D}\left(R^{2} v\right) \leq\left(1+\epsilon^{\prime}\right) J_{\tilde{\Omega}}(v) \quad \text { for all } v \in \Sigma_{\tilde{\Omega}} \\
& R^{2} v=v \quad \text { for all } v \in \Sigma_{\tilde{\Omega}} \cap H_{0}^{1}(O) .
\end{aligned}
$$

Step 3: Conclusion. 
Step 1: Existence of $R_{\lambda}^{1}$ satisfying (3.5), (3.6).

First we remark that $J_{2, \lambda}(v) \leq m$ implies

$$
\left(\frac{1}{2}-\frac{1}{p+1}\right)\left(\frac{\|v\|_{\lambda, \Omega_{2}^{\prime}, \#}}{\|v\|_{L^{p+1}\left(\Omega_{2}^{\prime}\right)}}\right)^{\frac{2(p+1)}{p-1}} \leq m .
$$

Recalling $\|v\|_{\lambda, \Omega_{2}^{\prime}, \#} \geq\|v\|_{\lambda, \Omega_{2}^{\prime}}=1$, we have

$$
\|v\|_{L^{p+1}\left(\Omega_{2}^{\prime}\right)} \geq \tilde{m} \equiv\left(\frac{\frac{1}{2}-\frac{1}{p+1}}{m}\right)^{\frac{p-1}{2(p+1)}} .
$$

The following proposition will be proved in Section 8, in which the subsolution estimate (cf. Simon 22]) plays an important role.

Proposition 3.4. For any $\tilde{m}>0, \tilde{\epsilon}>0$ and any open sets $\tilde{\Omega}$, $\tilde{\tilde{\Omega}}$ with smooth boundaries and $\Omega_{2} \subset \subset \tilde{\tilde{\Omega}} \subset \subset \tilde{\Omega} \subset \subset \Omega_{2}^{\prime}$, there exists $\lambda(\tilde{m}, \tilde{\epsilon}, \tilde{\Omega}, \tilde{\tilde{\Omega}}) \geq 1$ with the following properties: For any $\lambda \geq \lambda(\tilde{m}, \tilde{\epsilon}, \tilde{\Omega}, \tilde{\tilde{\Omega}})$ there exists an odd continuous mapping

$$
F_{\lambda}:\left\{v \in \Sigma_{2, \lambda} ;\|v\|_{L^{p+1}\left(\Omega_{2}^{\prime}\right)} \geq \tilde{m}\right\} \rightarrow H_{0}^{1}(\tilde{\Omega})
$$

such that for any $v \in\left\{v \in \Sigma_{2, \lambda} ;\|v\|_{L^{p+1}\left(\Omega_{2}^{\prime}\right)} \geq \tilde{m}\right\}$,

$1^{\circ}\left(F_{\lambda} v\right)(x)=v(x)$ for all $x \in \tilde{\tilde{\Omega}}$.

$2^{\circ}\left\|F_{\lambda} v\right\|_{\lambda, \Omega_{2}^{\prime}} \leq 1+\tilde{\epsilon}$.

$3^{\circ}\left\|F_{\lambda} v\right\|_{L^{p+1}\left(\Omega_{2}^{\prime}\right)} \geq(1-\tilde{\epsilon})\|v\|_{L^{p+1}\left(\Omega_{2}^{\prime}\right)}$.

Now we can show Step 1. Let $F_{\lambda}$ be an operator given in the above proposition. We define $R_{\lambda}^{1}$ by

$$
R_{\lambda}^{1} v=\frac{F_{\lambda} v}{\left\|F_{\lambda} v\right\|_{H^{1}(\tilde{\Omega})}} .
$$

By $1^{\circ}-3^{\circ}$ of Proposition 3.4 and (2.28),

$$
\begin{aligned}
J_{\tilde{\Omega}}\left(R_{\lambda}^{1} v\right) & =\left(\frac{1}{2}-\frac{1}{p+1}\right)\left(\frac{1}{\left\|R_{\lambda}^{1} v\right\|_{L^{p+1}\left(\Omega_{2}^{\prime}\right)}}\right)^{\frac{2(p+1)}{p-1}} \\
& \leq\left(\frac{1}{2}-\frac{1}{p+1}\right)\left(\frac{\left\|R_{\lambda}^{1} v\right\|_{\lambda, \Omega_{2}^{\prime}}}{\left\|R_{\lambda}^{1} v\right\|_{L^{p+1}\left(\Omega_{2}^{\prime}\right)}}\right)^{\frac{2(p+1)}{p-1}} \\
& =\left(\frac{1}{2}-\frac{1}{p+1}\right)\left(\frac{\left\|F_{\lambda} v\right\|_{\lambda, \Omega_{2}^{\prime}}}{\left\|F_{\lambda} v\right\|_{L^{p+1}\left(\Omega_{2}^{\prime}\right)}}\right)^{\frac{2(p+1)}{p-1}} \\
& \leq\left(\frac{1+\tilde{\epsilon}}{1-\tilde{\epsilon}}\right)^{\frac{2(p+1)}{p-1}}\left(\frac{1}{2}-\frac{1}{p+1}\right)\left(\frac{1}{\|v\|_{L^{p+1}\left(\Omega_{2}^{\prime}\right)}}\right)^{\frac{2(p+1)}{p-1}} \\
& \leq\left(\frac{1+\tilde{\epsilon}}{1-\tilde{\epsilon}}\right)^{\frac{2(p+1)}{p-1}} J_{2, \lambda}(v) \quad \text { for all } v \in \Sigma_{2, \lambda} .
\end{aligned}
$$

Thus we have (3.5) for sufficiently small $\tilde{\epsilon}>0$. (3.6) also clearly holds. 
Step 2: Existence of $\tilde{\Omega}$ and $R^{2}$ satisfying (3.7), (3.8).

Since $\partial \Omega_{2}$ is smooth, for any $\nu>0$ and $O \subset \subset \Omega_{2}$ we can find a smooth open set $\Omega_{2}^{(\nu)}$ satisfying $\Omega_{2} \subset \subset \Omega_{2}^{(\nu)} \subset \subset \Omega_{2}^{\prime}$ and a diffeomorphism $\varphi^{(\nu)}=\left(\varphi_{1}^{(\nu)}, \cdots, \varphi_{N}^{(\nu)}\right)$ : $\overline{\Omega_{2}} \rightarrow \overline{\Omega_{2}^{(\nu)}}$ such that

$$
\begin{aligned}
& \varphi^{(\nu)}(x)=x \quad \text { for all } x \in O, \\
& \max _{x \in \overline{\Omega_{2}}}\left|\frac{\partial \varphi_{i}^{(\nu)}}{\partial x_{j}}(x)-\delta_{i j}\right|<\nu \quad \text { for } i, j \in\{1,2, \cdots, N\} .
\end{aligned}
$$

We remark that $\Omega_{2}^{(\nu)}$ approaches $\Omega_{2}$ as $\nu \rightarrow 0$.

We define $\widetilde{\varphi}^{(\nu)}: H_{0}^{1}\left(\Omega_{2}^{(\nu)}\right) \rightarrow H_{0}^{1}\left(\Omega_{2}\right)$ by $\left(\widetilde{\varphi}^{(\nu)} u\right)(x)=u\left(\varphi^{(\nu)}(x)\right)$. We can observe that there exists a constant $c_{\nu}>0$ such that for $u \in H_{0}^{1}\left(\Omega_{2}^{(\nu)}\right)$

$$
\begin{aligned}
& c_{\nu} \rightarrow 0 \quad \text { as } \nu \rightarrow 0, \\
& \left(1-c_{\nu}\right)\|u\|_{L^{p+1}\left(\Omega_{2}^{(\nu)}\right)} \leq\left\|\widetilde{\varphi}^{(\nu)} u\right\|_{L^{p+1}\left(\Omega_{2}\right)} \leq\left(1+c_{\nu}\right)\|u\|_{L^{p+1}\left(\Omega_{2}^{(\nu)}\right)}, \\
& \left(1-c_{\nu}\right)\|u\|_{H^{1}\left(\Omega_{2}^{(\nu)}\right)} \leq\left\|\widetilde{\varphi}^{(\nu)} u\right\|_{H^{1}\left(\Omega_{2}\right)} \leq\left(1+c_{\nu}\right)\|u\|_{H^{1}\left(\Omega_{2}^{(\nu)}\right)} .
\end{aligned}
$$

Now we can prove (3.7). For a given $\epsilon>0$, we choose $\nu \in(0,1)$ such that $\left(\frac{1+c_{\nu}}{1-c_{\nu}}\right)^{\frac{2(p+1)}{p-1}}<1+\epsilon$. We will show that (3.7) holds for $\tilde{\Omega}=\Omega_{2}^{(\nu)}$.

For $v \in \Sigma_{\tilde{\Omega}}$ we define $R^{2} v \in \Sigma_{2, D}$ by $R^{2} v=\frac{\tilde{\varphi}^{(\nu)} v}{\left\|\tilde{\varphi}^{(\nu)} v\right\|_{H^{1}\left(\Omega_{2}\right)}}$. Then by (3.10)-(3.11)

$$
\begin{aligned}
J_{2, D}\left(R^{2} v\right) & =\left(\frac{1}{2}-\frac{1}{p+1}\right)\left(\frac{\left\|\tilde{\varphi}^{(\nu)} v\right\|_{H^{1}\left(\Omega_{2}\right)}}{\left\|\tilde{\varphi}^{(\nu)} v\right\|_{L^{p+1}\left(\Omega_{2}\right)}}\right)^{\frac{2(p+1)}{p-1}} \\
& \leq\left(\frac{1+c_{\nu}}{1-c_{\nu}}\right)^{\frac{2(p+1)}{p-1}}\left(\frac{1}{2}-\frac{1}{p+1}\right)\left(\frac{\|v\|_{H^{1}\left(\Omega_{2}^{(\nu)}\right)}}{\|v\|_{L^{p+1}\left(\Omega_{2}^{(\nu)}\right)}}\right)^{\frac{2(p+1)}{p-1}} \\
& =\left(\frac{1+c_{\nu}}{1-c_{\nu}}\right)^{\frac{2(p+1)}{p-1}} J_{\tilde{\Omega}}(v) .
\end{aligned}
$$

By the choice of $\nu$, we have (3.7). Equation (3.8) also follows from (3.9).

Step 3: Conclusion.

For a given $\delta>0$ we choose $\epsilon, \epsilon^{\prime}>0$ such that $(1+\epsilon)\left(1+\epsilon^{\prime}\right)<1+\delta$. By Step 2, there exist $\tilde{\Omega}$ and $R^{2}$ such that (3.7) holds. Next, using Step 1, we find $\lambda_{\tilde{\Omega}, \epsilon, m} \geq 1$ and $R_{\lambda}^{1}$ $\left(\lambda \geq \lambda_{\tilde{\Omega}, \epsilon, m}\right)$ such that (3.5) holds. Defining $R_{\lambda}:\left\{v \in \Sigma_{2, \lambda} ; J_{2, \lambda}(v) \leq m\right\} \rightarrow \Sigma_{2, D}$ by $R_{\lambda}=R^{2} \circ R_{\lambda}^{1}$, we have (3.3)- (3.4).

As stated in the Introduction, since $b_{n}^{2, D} \rightarrow \infty$ as $n \rightarrow \infty$, there exists a sequence $n(1)<n(2)<\cdots<n(k)<n(k+1)<\cdots$ such that

$$
b_{n(k)}^{2, D}<b_{n(k)+1}^{2, D} .
$$

We set

$$
\delta_{k}=\frac{1}{4}\left(b_{n(k)+1}^{2, D}-b_{n(k)}^{2, D}\right)>0
$$


and choose $\gamma_{0 k} \in \Gamma_{n(k)}^{2, D}$ such that

$$
\begin{aligned}
& \max _{\theta \in S^{n(k)}} J_{2, D}\left(\gamma_{0 k}(\theta)\right)<b_{n(k)}^{2, D}+\delta_{k}, \\
& \text { supp } \gamma_{0 k} \subset \Omega_{2} \text { is compact in } \Omega_{2} \text { for all } \theta \in S^{n(k)} .
\end{aligned}
$$

We define a minimax value $c_{k}^{2, D}$ by

$$
c_{k}^{2, D}=\inf _{\sigma \in \Lambda_{k}^{2, D}} \max _{\theta \in S_{+}^{n(k)+1}} J_{2, D}(\sigma(\theta)),
$$

where

$$
\Lambda_{k}^{2, D}=\left\{\sigma \in C\left(S_{+}^{n(k)+1}, \Sigma_{2, D}\right) ; \sigma(\theta)=\gamma_{0 k}(\theta) \text { for all } \theta \in S^{n(k)}\right\} .
$$

Here we regard $S^{n(k)}=\left\{\left(\theta_{1}, \cdots, \theta_{n(k)+1}, 0\right) ; \theta_{1}^{2}+\cdots+\theta_{n(k)+1}^{2}=1\right\}=\partial S_{+}^{n(k)+1} \subset$ $S_{+}^{n(k)+1}$.

This type of minimax method is introduced in Ambrosetti [1, Bahri-Berestycki [5], Struwe 23] and Rabinowitz [20. In the definition (3.16)-(3.17) of $c_{k}^{2, D}$ we don't require evenness, and it enables us to detect critical points of perturbed functionals.

As a fundamental property of $c_{k}^{2, D}$ we have

Lemma 3.5. $\quad$ (i) $c_{k}^{2, D} \geq b_{n(k)+1}^{2, D}\left(>b_{n(k)}^{2, D}\right)$.

(ii) $c_{k}^{2, D}$ is a critical value of $J_{2, D}\left(v_{2}\right)$.

Proof. (i) For a given $\sigma \in \Lambda_{k}^{2, D}$ we define $\gamma_{\sigma} \in \Gamma_{n(k)+1}^{2, D}$ by

$$
\gamma_{\sigma}(\theta)= \begin{cases}\sigma(\theta) & \text { if } \theta \in S_{+}^{n(k)+1}, \\ -\sigma(-\theta) & \text { if } \theta \in S^{n(k)+1} \backslash S_{+}^{n(k)+1} .\end{cases}
$$

Since $J_{2, D}$ is even, we have $\max _{\theta \in S^{n(k)+1}} J_{2, D}\left(\gamma_{\sigma}(\theta)\right)=\max _{\theta \in S_{+}^{n(k)+1}} J_{2, D}(\sigma(\theta))$. Thus we have $c_{k}^{2, D} \geq b_{n(k)+1}^{2, D}$.

(ii) By (i), we can see that $\Lambda_{k}^{2, D}$ is stable under a suitable class of deformations. Thus we can observe that $c_{k}^{2, D}$ is a critical point value of $J_{2, D}\left(v_{2}\right)$.

Next we introduce related minimax methods to $J_{2, \lambda}\left(v_{2}\right)$. By Proposition [3.2, (3.1), and (3.12), for any $k \in \mathbf{N}$ there exists $\lambda(k)$ such that for $\lambda \geq \lambda(k)$

$$
b_{n(k)}^{2, \lambda} \leq b_{n(k)}^{2, D} \leq b_{n(k)}^{2, \lambda}+\delta_{k}, \quad b_{n(k)}^{2, \lambda} \leq b_{n(k)+1}^{2, \lambda}-3 \delta_{k} .
$$

We define for $\lambda \geq \lambda(k)$

$$
c_{k}^{2, \lambda}=\inf _{\sigma \in \Lambda_{k}^{2, \lambda}} \max _{\theta \in S_{+}^{n(k)+1}} J_{2, \lambda}(\sigma(\theta))
$$

where $\Lambda_{k}^{2, \lambda}=\left\{\sigma \in C\left(S_{+}^{n(k)+1}, \Sigma_{2, \lambda}\right) ; \sigma(\theta)=\gamma_{0 k}(\theta)\right.$ for all $\left.\theta \in S^{n(k)}\right\}$. Here $\gamma_{0 k} \in$ $\Gamma_{n(k)}^{2, D} \subset \Gamma_{n(k)}^{2, \lambda}$ is chosen in (3.14)-(3.15).

As in Lemma 3.5 and Proposition 3.2, we have

Lemma 3.6. $\quad$ (i) $c_{k}^{2, \lambda} \geq b_{n(k)+1}^{2, \lambda}$ for $\lambda \geq \lambda(k)$.

(ii) $c_{k}^{2, \lambda}$ is a critical value of $J_{2, \lambda}\left(v_{2}\right)$ for $\lambda \geq \lambda(k)$.

(iii) $c_{k}^{2, \lambda} \rightarrow c_{k}^{2, D}$ as $\lambda \rightarrow \infty$. 
Proof. (i) As in (i) of Lemma 3.5, we have (i).

(ii) By (3.14) and (3.18),

$$
\begin{aligned}
\max _{\theta \in S^{n(k)}} J_{2, \lambda}\left(\gamma_{0 k}(\theta)\right) & =\max _{\theta \in S^{n(k)}} J_{2, D}\left(\gamma_{0 k}(\theta)\right)<b_{n(k)}^{2, D}+\delta_{k} \\
& \leq b_{n(k)}^{2, \lambda}+2 \delta_{k} \leq b_{n(k)+1}^{2, \lambda}-\delta_{k}<c_{k}^{2, \lambda} .
\end{aligned}
$$

Thus we can see that $c_{k}^{2, \lambda}$ is a critical value of $J_{2, \lambda}\left(v_{2}\right)$.

(iii) We recall (3.15) and choose $O \subset \subset \Omega_{2}$ such that $\overline{\bigcup_{\theta \in S^{n(k)}} \operatorname{supp} \gamma_{0 k}(\theta)} \subset O$. Applying Lemma 3.3. we can show (iii) as in Proposition 3.2

(b) Minimax methods for $J_{\lambda}\left(v_{1}, v_{2}\right)$. Let $(n(k))_{k=1}^{\infty},\left(\gamma_{0 k}\right)_{k=1}^{\infty}$ be sequences obtained in (3.12) -3.15) and let $v_{10} \in \Sigma_{1, D}$ be a minimizer of $J_{1, D}\left(v_{1}\right)$, that is,

$$
J_{1, D}\left(v_{10}\right)=c_{\text {min }}^{1, D} .
$$

We remark that we may assume $v_{10}>0$ in $\Omega_{1}$. Using $(n(k))_{k=1}^{\infty},\left(\gamma_{0 k}\right)_{k=1}^{\infty}$ and $v_{10}$, we introduce minimax values $c_{k}^{\lambda}$ for $J_{\lambda}\left(v_{1}, v_{2}\right)$ by

$$
c_{k}^{\lambda}=\inf _{\tilde{\gamma} \in \Lambda_{k}^{\lambda}} \max _{\theta \in S_{+}^{n(k)+1}} J_{\lambda}(\tilde{\gamma}(\theta)),
$$

where

$$
\begin{aligned}
\Lambda_{k}^{\lambda}=\left\{\tilde{\gamma}=\left(\tilde{\gamma}_{1}, \tilde{\gamma}_{2}\right) \in\right. & C\left(S_{+}^{n(k)+1}, \Sigma_{1, \lambda} \times \Sigma_{2, \lambda}\right) ; \\
& \tilde{\gamma}(\theta)=\left(v_{10}, \gamma_{0 k}(\theta)\right) \text { for all } \theta \in S^{n(k)}, \\
& \left.\left\|\tilde{\gamma}_{1}(\theta)_{-}\right\|_{L^{p+1}\left(\Omega_{1}^{\prime}\right)}^{p+1} \leq \nu_{M_{k}} \text { for all } \theta \in S_{+}^{n(k)+1}\right\} .
\end{aligned}
$$

Here $M_{k}=c_{\text {min }}^{1, D}+c_{k}^{2, D}+1>0$ and $\nu_{M_{k}}>0$ is given in Proposition 9.1 and Corollary 9.3 in Section 9 , First we remark

$$
c_{k}^{\lambda} \leq c_{\min }^{1, D}+c_{k}^{2, D} \quad \text { for all } \lambda \geq 1 .
$$

In fact, for any $\epsilon>0$ there exists $\gamma_{\epsilon} \in \Lambda_{k}^{2, D}$ such that $\max _{\theta \in S_{+}^{n(k)+1}} J_{2, D}\left(\gamma_{\epsilon}(\theta)\right)<$ $c_{k}^{2, D}+\epsilon$. Setting $\tilde{\gamma}_{\epsilon}(\theta)=\left(v_{10}, \gamma_{\epsilon}(\theta)\right) \in \Lambda_{k}^{\lambda}$ and noting $J_{\lambda}\left(\tilde{\gamma}_{\epsilon}(\theta)\right)=J_{\lambda}\left(v_{10}, \gamma_{\epsilon}(\theta)\right)=$ $J_{1, D}\left(v_{10}\right)+J_{2, D}\left(\gamma_{\epsilon}(\theta)\right)$, we have $c_{k}^{\lambda} \leq c_{\text {min }}^{1, D}+c_{k}^{2, D}+\epsilon$ for all $\lambda \geq 1$. Since $\epsilon>0$ is arbitrary, we have (3.19). We choose $\lambda_{M_{k}} \geq 1$ such that (2.32) holds for $\lambda \geq \lambda_{M_{k}}$ and $v \in\left[J_{\lambda} \leq M_{k}\right]_{\Sigma_{1, \lambda} \times \Sigma_{2, \lambda}}$.

Now we can prove

Proposition 3.7. $\quad$ (i) For $\lambda \geq \lambda_{M_{k}}$,

$$
c_{k}^{\lambda} \geq c_{\min }^{1, \lambda}+c_{k}^{2, \lambda}-r_{\lambda} .
$$

$$
\lim _{\lambda \rightarrow \infty} c_{k}^{\lambda}=c_{\min }^{1, D}+c_{k}^{2, D} .
$$

(iii) For sufficiently large $\lambda, c_{k}^{\lambda}$ is a critical value of $J_{\lambda}\left(v_{1}, v_{2}\right)$.

Proof. (i) For any $\tilde{\gamma}(\theta)=(p(\theta), \gamma(\theta)) \in \Lambda_{k}^{\lambda}$, we observe $\gamma(\theta) \in \Lambda_{k}^{2, \lambda}$. Thus for any $\tilde{\gamma} \in \Lambda_{k}^{\lambda}$ satisfying $\max _{\theta \in S_{+}^{n(k)+1}} J_{\lambda}(\tilde{\gamma}(\theta)) \leq M_{k}$, we have by (2.32) that

$J_{\lambda}(\tilde{\gamma}(\theta)) \geq J_{1, \lambda}(p(\theta))+J_{2, \lambda}(\gamma(\theta))-r_{\lambda} \geq c_{m i n}^{1, \lambda}+J_{2, \lambda}(\gamma(\theta))-r_{\lambda} \quad$ for all $\theta \in S_{+}^{n(k)+1}$.

Thus we get (3.20). 
(ii) (3.21) follows easily from (3.19), (3.20), (i) of Proposition 3.2 and (iii) of Lemma 3.6

(iii) For $\theta \in S^{n(k)}$, we have

$$
J_{\lambda}\left(v_{10}, \gamma_{0 k}(\theta)\right)=J_{1, \lambda}\left(v_{10}\right)+J_{2, \lambda}\left(\gamma_{0 k}(\theta)\right) \leq c_{m i n}^{1, D}+b_{n(k)}^{2, D}+\delta_{k} .
$$

Thus we have from (3.21), (3.13) and (i) of Lemma 3.5 that

$$
\max _{\theta \in S^{n(k)}} J_{\lambda}\left(v_{10}, \gamma_{0 k}(\theta)\right) \leq c_{k}^{\lambda}-2 \delta_{k}
$$

for sufficiently large $\lambda$, and we can show $\Lambda_{k}^{\lambda}$ is stable under a suitable class of deformations. Thus $c_{k}^{\lambda}$ is a critical value of $J_{\lambda}\left(v_{1}, v_{2}\right)$ for sufficiently large $\lambda$.

At this stage we remark that if critical point $v_{\lambda}=\left(v_{1 \lambda}, v_{2 \lambda}\right) \in \Sigma_{1, \lambda} \times \Sigma_{2, \lambda}$ satisfies

$$
\begin{aligned}
& J_{\lambda}\left(v_{\lambda}\right) \rightarrow c_{\min }^{1, D}+c_{k}^{2, D}, \\
& \left\|\left(v_{1 \lambda}\right)_{-}\right\|_{L^{p+1}\left(\Omega_{1}^{\prime}\right)}^{p+1}<\delta_{0}\left(M_{k}\right),
\end{aligned}
$$

then by Proposition 2.17 and Proposition 2.19 a sequence of solutions $u_{\lambda}(x)$ corresponding to $v_{\lambda}$ satisfies (1.10) 1.13 . We remark that by Corollary 9.3 in Section 9 there exists a critical point $v_{\lambda}$ corresponding to the critical value $c_{k}^{\lambda}$ and $v_{\lambda}$ satisfies (3.22) - (3.23).

In the next section we show (1.14).

\section{Proof of Theorem 1.1}

(a) Construction of a pseudo-gradient vector field. By the argument in the previous sections, to prove Theorem 1.1 we need to show (1.14). For that purpose, we need to construct a deformation flow $\eta(t)$ under which the level set $\left\{v_{1} \in \Sigma_{1, \lambda} ; J_{1, \lambda}\left(v_{1}\right) \leq c_{m i n}^{1, D}+\delta\right\}$ is invariant for small $\delta>0$. The following isolatedness of critical values is important for the construction of such a flow.

Proposition 4.1 ([Dancer [12, 13]; cf. Cao-Noussair [10]). Let

$$
\widetilde{\Psi}_{1, D}(u)=\int_{\Omega_{1}} \frac{1}{2}\left(|\nabla u|^{2}+u^{2}\right)-\frac{1}{p+1} u_{+}^{p+1} d x: H_{0}^{1}\left(\Omega_{1}\right) \rightarrow \mathbf{R}
$$

and suppose that $u(x)$ is a positive solution of (1.4) in $\Omega_{1}$. Then $\widetilde{\Psi}_{1, D}(u)$ is an isolated critical value of $\widetilde{\Psi}_{1, D}$.

Proof. See the Appendix of [10] and [12, 13]. Here the analyticity of the map $u \mapsto(-\Delta)^{-1}\left(u^{p}\right)$ in $C^{1}(\Omega)$ near a positive solution is essential.

By Proposition 4.1, we can see $c_{\text {min }}^{1, D}$ is an isolated critical value of $\Psi_{1, D}(u)$ (equivalently of $J_{1, D}(v)$ ).

Remark 4.2. For the following arguments, we need only nowhere denseness of critical values in a neighborhood of $c_{\text {min }}^{1, D}$ or $c_{k}^{2, D}$. We remark that isolatedness implies nowhere denseness. We refer to Fučík-Kučera-Nečas-Souček-Souček [17] for the Morse-Sard theorem in infinite dimensional setting. We also remark that Sard's property is also used effectively in the study of the Hamiltonian system in Cieliebak-Séré [11]. 
First we have

Lemma 4.3. Suppose that for $v \in \Sigma_{1, D}$

$$
J_{1, D}(v) \in[\alpha, \beta] \quad \text { implies } \quad J_{1, D}^{\prime}(v) \neq 0
$$

for some $0<\alpha<\beta<\infty$. Then there exist $\rho_{0}>0$ and $\lambda_{0} \geq 1$ such that for $\lambda \geq \lambda_{0}$, $v \in \Sigma_{1, \lambda}$

$$
J_{1, \lambda}(v) \in[\alpha, \beta] \quad \text { implies }\left\|J_{1, \lambda}^{\prime}(v)\right\|_{T_{v}\left(\Sigma_{1, \lambda}\right)^{*}} \geq \rho_{0} .
$$

Proof. We argue indirectly, and we assume that there exist $\lambda_{n} \geq 1, v_{n} \in \Sigma_{1, \lambda_{n}}$ $(n=1,2, \cdots)$ satisfying

$$
\lambda_{n} \rightarrow \infty, \quad J_{1, \lambda_{n}}\left(v_{n}\right) \rightarrow c \in[\alpha, \beta], \quad\left\|J_{1, \lambda_{n}}^{\prime}\left(v_{n}\right)\right\|_{T_{v_{n}}\left(\Sigma_{1, \lambda_{n}}\right)^{*}} \rightarrow 0 \quad \text { as } n \rightarrow \infty .
$$

By Lemma 2.18, after extracting a subsequence — still denoted by $n-$, there exists $v_{\infty} \in \Sigma_{1, D}$ such that (2.40)-(2.41) hold. In particular, we have that $c$ is a critical value of $J_{1, D}(v)$, which is in contradiction to assumption (4.1).

We also have

Lemma 4.4. Suppose that (4.1) holds. Then for any $M>0$ there exists $\lambda_{1}(M) \geq$ $\lambda_{0}$ such that for any $\lambda \geq \lambda_{1}(M)$ and $v=\left(v_{1}, v_{2}\right) \in \Sigma_{1, \lambda} \times \Sigma_{2, \lambda}$ satisfying $J_{\lambda}\left(v_{1}, v_{2}\right)$ $\leq M$ and $J_{1, \lambda}\left(v_{1}\right) \in[\alpha, \beta]$, there exists

$$
X=\left(X_{1}, X_{2}\right) \in T_{v}\left(\Sigma_{1, \lambda} \times \Sigma_{2, \lambda}\right)=\left(T_{v_{1}}\left(\Sigma_{1, \lambda}\right)\right) \oplus\left(T_{v_{2}}\left(\Sigma_{2, \lambda}\right)\right)
$$

such that

$$
\begin{aligned}
& \left\|X_{1}\right\|_{T_{v_{1}}\left(\Sigma_{1, \lambda}\right)}=1, \quad X_{2}=0, \\
& J_{1, \lambda}^{\prime}\left(v_{1}\right) X_{1} \geq \frac{\rho_{0}}{2}, \quad J_{\lambda}^{\prime}\left(v_{1}, v_{2}\right)\left(X_{1}, X_{2}\right) \geq \frac{\rho_{0}}{2},
\end{aligned}
$$

where $\rho_{0}>0$ is a constant appearing in (4.2).

Proof. This is a consequence from (4.2) and (2.32)-(2.33).

To prove Theorem 1.1, it suffices to show for any $\delta>0$ there exists a critical point $v_{\lambda}=\left(v_{1 \lambda}, v_{2 \lambda}\right) \in \Sigma_{1, \lambda} \times \Sigma_{2, \lambda}$ of $J_{\lambda}(v)$ for sufficiently large $\lambda$ with the following properties:

$$
\left|J_{1, \lambda}\left(v_{1 \lambda}\right)-c_{\min }^{1, D}\right|<\delta, \quad\left|J_{2, \lambda}\left(v_{2 \lambda}\right)-c_{k}^{2, D}\right|<\delta
$$

In fact, if the above statement is true, then there exists a sequence $v_{\lambda}=\left(v_{1 \lambda}, v_{2 \lambda}\right)$ such that

$$
J_{1, \lambda}\left(v_{1 \lambda}\right) \rightarrow c_{\min }^{1, D}, \quad J_{2, \lambda}\left(v_{2 \lambda}\right) \rightarrow c_{k}^{2, D} .
$$

Clearly it follows from (4.4) that

$$
J_{\lambda}\left(v_{\lambda}\right) \rightarrow c_{\min }^{1, D}+c_{k}^{2, D} \quad \text { as } \lambda \rightarrow \infty .
$$

Thus by Proposition 2.17, after extracting a subsequence, we have $v_{i \lambda_{n}} \rightarrow v_{i \infty}$ $(i=1,2)$ and

$$
J_{1, D}\left(v_{1 \infty}\right)=\lim _{n \rightarrow \infty} J_{1, \lambda_{n}}\left(v_{1 \lambda_{n}}\right)=c_{\min }^{1, D} .
$$

Thus $v_{1 \infty}(x)$ has a constant sign, and we may assume $v_{1 \infty}(x)>0$ and Theorem 1.1 follows. We remark here that critical point $v_{\lambda}(x)$ obtained in this section may not satisfy $J_{\lambda}\left(v_{\lambda}\right)=c_{k}^{\lambda}$. 
In what follows, we argue indirectly and assume for some $\delta_{0}>0$ that

there are no critical points $v=\left(v_{1}, v_{2}\right) \in \Sigma_{1, \lambda} \times \Sigma_{2, \lambda}$ of $J_{\lambda}(v)$ satisfying (4.3) with $\delta=\delta_{0}$.

For $\epsilon_{0} \in\left(0, \delta_{0}\right)$ we choose a neighborhood $N_{\epsilon_{0}, \lambda}$ of $\left(c_{m i n}^{1, D}, c_{k}^{2, D}\right)$ in $\mathbf{R}^{2}$ in the following way: By the isolatedness of $c_{\text {min }}^{1, D}$, we can choose an interval $[\alpha, \beta]$ such that

$$
[\alpha, \beta] \subset\left(c_{\text {min }}^{1, D}+\frac{1}{2} \delta_{0}, c_{\text {min }}^{1, D}+\delta_{0}\right)
$$

and $J_{1, D}\left(v_{1}\right)$ has no critical values in $[\alpha, \beta]$. We set

$$
\begin{aligned}
N_{\epsilon_{0}, \lambda}= & \left\{\left(j_{1}, j_{2}\right) \in \mathbf{R}^{2} ; c_{\text {min }}^{1, D}-\frac{1}{2} \delta_{0} \leq j_{1} \leq \beta\right. \\
& \left(1+r_{\lambda}\right)^{-\frac{2(p+1)}{p-1}}\left(c_{\text {min }}^{1, \lambda}+c_{k}^{2, \lambda}-\epsilon_{0}\right) \leq j_{1}+j_{2} \\
& \left.\leq\left(1-r_{\lambda}\right)^{-\frac{2(p+1)}{p-1}}\left(c_{\text {min }}^{1, \lambda}+c_{k}^{2, \lambda}+\epsilon_{0}\right)\right\} .
\end{aligned}
$$

Since $c_{\min }^{1, \lambda} \rightarrow c_{\min }^{1, D}, c_{k}^{2, \lambda} \rightarrow c_{k}^{2, D}$ as $\lambda \rightarrow \infty$, we can see for $\epsilon_{0}>0$ small and $\lambda>0$ large

$$
\begin{aligned}
& \left(c_{\min }^{1, D}, c_{k}^{2, D}\right) \in N_{\epsilon_{0}, \lambda}, \\
& N_{\epsilon_{0}, \lambda} \subset\left(c_{\min }^{1, D}-\delta_{0}, c_{\min }^{1, D}+\delta_{0}\right) \times\left(c_{k}^{2, D}-\delta_{0}, c_{k}^{2, D}+\delta_{0}\right), \\
& 0<\epsilon_{0}<\delta_{k}=\frac{1}{4}\left(b_{n(k)+1}^{2, D}-b_{n(k)}^{2, D}\right) .
\end{aligned}
$$

By assumption (4.5), we have the following.

Lemma 4.5. Let

$$
A_{\lambda}=\left\{\left(v_{1}, v_{2}\right) \in \Sigma_{1, \lambda} \times \Sigma_{2, \lambda} ;\left(J_{1, \lambda}\left(v_{1}\right), J_{2, \lambda}\left(v_{2}\right)\right) \in N_{\epsilon_{0}, \lambda}\right\},
$$

where $\epsilon_{0}>0$ is fixed so that (4.6)-(4.8) hold. Then for sufficiently large $\lambda$ there exists a vector field $X$ on $A_{\lambda}$ such that

(i) $X(v)=\left(X_{1}(v), X_{2}(v)\right) \in T_{v}\left(\Sigma_{1, \lambda} \times \Sigma_{2, \lambda}\right),\|X(v)\|_{T_{v}\left(\Sigma_{1, \lambda} \times \Sigma_{2, \lambda}\right)} \in\left[\frac{1}{2}, 1\right]$ for all $v \in A_{\lambda}$ and $v \mapsto X(v)$ is locally Lipschitz continuous.

(ii) There is a constant $\mu_{\lambda}>0$ such that $J_{\lambda}^{\prime}(v) X(v) \geq \mu_{\lambda}$ for all $v \in A_{\lambda}$.

(iii) If $v=\left(v_{1}, v_{2}\right) \in A_{\lambda}$ satisfies $J_{1, \lambda}\left(v_{1}\right) \in[\alpha, \beta]$, then $X(v)=\left(X_{1}(v), 0\right)$ and it satisfies $J_{1, \lambda}^{\prime}\left(v_{1}\right) X_{1}(v) \geq \frac{\rho_{0}}{2}$ and $J_{\lambda}^{\prime}(v) X(v) \geq \frac{\rho_{0}}{2}$.

Proof. First we remark that $v=\left(v_{1}, v_{2}\right) \in A_{\lambda}$ implies $J_{1, \lambda}\left(v_{1}\right) \leq c_{\text {min }}^{1, D}+\delta_{0}$, $J_{2, \lambda}\left(v_{2}\right) \leq c_{k}^{2, D}+\delta_{0}$. Thus by (i) of Lemma 2.14, we have

$$
J_{\lambda}(v) \leq\left(1+r_{\lambda}\right)^{\frac{2(p+1)}{p-1}}\left(c_{\min }^{1, D}+c_{k}^{2, D}+2 \delta_{0}\right)
$$

Choosing $M=2^{\frac{2(p+1)}{p-1}}\left(c_{\min }^{1, D}+c_{k}^{2, D}+2 \delta_{0}\right)$, we can apply Lemma 4.4 in the setting (iii). We also remark that $J_{\lambda}(v)$ satisfies the Palais-Smale condition. Thus under assumption (4.5), we have $\inf _{v \in A_{\lambda}}\left\|J_{\lambda}^{\prime}(v)\right\|_{T_{v}\left(\Sigma_{1, \lambda} \times \Sigma_{2, \lambda}\right)^{*}}>0$. In a standard way, we can construct a locally Lipschitz continuous pseudo-gradient vector field satisfying (i)-(iii). 
Remark 4.6. It is easily seen that there exists a constant $\nu_{0}>0$ such that for large $\lambda$

(4.9) $v=\left(v_{1}, v_{2}\right) \in A_{\lambda} \quad$ implies

$$
\begin{aligned}
& \left\|J_{\lambda}^{\prime}(v)\right\|_{T_{v}\left(\Sigma_{1, \lambda} \times \Sigma_{2, \lambda}\right)^{*}},\left\|J_{1, \lambda}^{\prime}\left(v_{1}\right)\right\|_{T_{v_{1}}\left(\Sigma_{1, \lambda}\right)^{*}} \\
& \left\|J_{2, \lambda}^{\prime}\left(v_{2}\right)\right\|_{T_{v_{2}}\left(\Sigma_{2, \lambda}\right)^{*}} \leq \nu_{0} \\
& J_{1, \lambda}\left(v_{1}\right) \geq c_{\min }^{1, \lambda} \geq c_{\min }^{1, D}-\delta_{0}
\end{aligned}
$$

We also have the following.

Lemma 4.7. For sufficiently large $\lambda$, we have for all $v=\left(v_{1}, v_{2}\right) \in \Sigma_{1, \lambda} \times \Sigma_{2, \lambda}$

(i) $J_{1, \lambda}\left(v_{1}\right) \geq c_{\text {min }}^{1, \lambda}$.

(ii) $J_{1, \lambda}\left(v_{1}\right)+J_{2, \lambda}\left(v_{2}\right) \geq\left(1-r_{\lambda}\right)^{-\frac{2(p+1)}{p-1}}\left(c_{\text {min }}^{1, \lambda}+c_{k}^{2, \lambda}+\epsilon_{0}\right)$ implies $J_{\lambda}\left(v_{1}, v_{2}\right) \geq$ $c_{\text {min }}^{1, D}+c_{k}^{2, D}+\frac{2}{3} \epsilon_{0}$.

(iii) $J_{1, \lambda}\left(v_{1}\right)+J_{2, \lambda}\left(v_{2}\right) \leq\left(1+r_{\lambda}\right)^{-\frac{2(p+1)}{p-1}}\left(c_{\text {min }}^{1, \lambda}+c_{k}^{2, \lambda}-\epsilon_{0}\right)$ implies $J_{\lambda}\left(v_{1}, v_{2}\right) \leq$ $c_{\text {min }}^{1, D}+c_{k}^{2, D}-\frac{2}{3} \epsilon_{0}$.

Proof. (i) is obvious. (ii), (iii) follow from (i) of Lemma 2.14 since $c_{\text {min }}^{1, \lambda} \rightarrow c_{\text {min }}^{1, D}$, $c_{k}^{2, \lambda} \rightarrow c_{k}^{2, D}$ as $\lambda \rightarrow \infty$.

We choose a function $\psi \in C^{\infty}(\mathbf{R},[0,1])$ such that

$$
\psi(s)= \begin{cases}1 & \text { for } s \geq c_{\min }^{1, D}+c_{k}^{2, D}-\frac{1}{3} \epsilon_{0}, \\ 0 & \text { for } s \leq c_{\min }^{1, D}+c_{k}^{2, D}-\frac{2}{3} \epsilon_{0} .\end{cases}
$$

We define a vector field $\widetilde{X}$ on $A_{\lambda}$ :

$$
\widetilde{X}\left(v_{1}, v_{2}\right)=\psi\left(J_{\lambda}\left(v_{1}, v_{2}\right)\right) X\left(v_{1}, v_{2}\right)
$$

and consider the following $\mathrm{ODE}$ in $A_{\lambda}$ :

$$
\frac{d \eta}{d t}=-\tilde{X}(\eta), \quad \eta\left(0, u_{0}\right)=u_{0}
$$

(b) End of the proof of Theorem 1.1, To prove Theorem 1.1, we argue as in Section 3 and choose $\gamma_{\epsilon} \in \Lambda_{k}^{2, D}$ (defined in (3.17)) such that

$$
\max _{\theta \in S_{+}^{n(k)+1}} J_{2, D}\left(\gamma_{\epsilon}(\theta)\right)<c_{k}^{2, D}+\epsilon .
$$

We consider a deformation of $\tilde{\gamma}_{\epsilon}(\theta)=\left(v_{01}, \gamma_{\epsilon}(\theta)\right) \in \Lambda_{k}^{\lambda}$ through the deformation flow (4.10). In this section we write

$$
\begin{array}{r}
\Lambda_{k}^{\lambda}=\left\{\tilde{\gamma}=\left(\tilde{\gamma}_{1}, \tilde{\gamma}_{2}\right) \in C\left(S_{+}^{n(k)+1}, \Sigma_{1, \lambda} \times \Sigma_{2, \lambda}\right) ;\right. \\
\left.\tilde{\gamma}(\theta)=\left(v_{10}, \gamma_{0 k}(\theta)\right) \text { for all } \theta \in S^{n(k)}\right\} .
\end{array}
$$

Choosing $\epsilon \in\left(0, \epsilon_{0}\right)$ small, we have

$$
\begin{aligned}
J_{\lambda}\left(v_{01}, \gamma_{\epsilon}(\theta)\right) & =J_{1, \lambda}\left(v_{01}\right)+J_{2, \lambda}\left(\gamma_{\epsilon}(\theta)\right) \leq c_{\text {min }}^{1, D}+c_{k}^{2, D}+\epsilon \\
& \leq\left(1-r_{\lambda}\right)^{-\frac{2(p+1)}{p-1}}\left(c_{\text {min }}^{1, \lambda}+c_{k}^{2, \lambda}+\epsilon_{0}\right)
\end{aligned}
$$

for all $\theta \in S_{+}^{n(k)+1}$.

We will observe that

(i) $\tilde{\gamma}_{\epsilon}(\theta) \notin A_{\lambda}$ implies $J_{\lambda}\left(\tilde{\gamma}_{\epsilon}(\theta)\right)<c_{m i n}^{1, D}+c_{k}^{2, D}-\frac{2}{3} \epsilon_{0}$ (Lemma 4.8). 
(ii) For $\tilde{\gamma}_{\epsilon}(\theta) \in A_{\lambda}$, we consider $\eta(t)=\eta\left(t, \tilde{\gamma}_{\epsilon}(\theta)\right)$, where $\eta\left(t, u_{0}\right)$ is a solution of (4.10). Then

(a) $\frac{d}{d t} J_{\lambda}(\eta(t)) \leq 0$ for all $t \geq 0$.

(b) $J_{\lambda}(\eta(t)) \geq c_{\text {min }}^{1, D}+c_{k}^{2, D}-\frac{1}{3} \epsilon_{0}$ implies $J_{1, \lambda}(\eta(t)) \in\left[c_{\text {min }}^{1, D}-\delta_{0}, \beta\right)$ and $\frac{d}{d t} J_{\lambda}(\eta(t)) \leq-\mu_{\lambda}$ (Lemma 4.9).

First we have

Lemma 4.8. For sufficiently large $\lambda$

$$
J_{\lambda}\left(v_{01}, \gamma_{\epsilon}(\theta)\right) \geq c_{\min }^{1, D}+c_{k}^{2, D}-\frac{2}{3} \epsilon_{0}
$$

implies

$$
\left(v_{01}, \gamma_{\epsilon}(\theta)\right) \in A_{\lambda} .
$$

Proof. First we recall $J_{1, \lambda}\left(v_{01}\right)=c_{\text {min }}^{1, D}<\alpha<\beta$. Suppose that $\left(v_{01}, \gamma_{\epsilon}(\theta)\right)(\theta \in$ $S_{+}^{n(k)+1}$ ) satisfies (4.12). By (iii) of Lemma 4.7, we have

$$
J_{1, \lambda}\left(v_{01}\right)+J_{2, \lambda}\left(\gamma_{\epsilon}(\theta)\right) \geq\left(1+r_{\lambda}\right)^{-\frac{2(p+1)}{p-1}}\left(c_{\text {min }}^{1, \lambda}+c_{k}^{2, \lambda}-\epsilon_{0}\right) .
$$

Thus recalling (4.11), we can see (4.13) holds for all $\theta \in S_{+}^{n(k)+1}$ satisfying (4.12).

Now we define for $\theta \in S_{+}^{n(k)+1}$ and $L>0$

$$
\widetilde{\gamma}_{L}(\theta)= \begin{cases}\left(v_{01}, \gamma_{\epsilon}(\theta)\right) & \text { if }\left(v_{01}, \gamma_{\epsilon}(\theta)\right) \notin A_{\lambda}, \\ \eta\left(L,\left(v_{01}, \gamma_{\epsilon}(\theta)\right)\right) & \text { if }\left(v_{01}, \gamma_{\epsilon}(\theta)\right) \in A_{\lambda},\end{cases}
$$

where $\eta\left(t, u_{0}\right)$ is a solution of (4.10).

The following lemma is the key of the proof; in particular it ensures $\widetilde{\gamma}_{L}(\theta)$ is well-defined for all $L>0$ provided $\epsilon>0$ is sufficiently small.

Lemma 4.9. Suppose that $\left(v_{01}, \gamma_{\epsilon}(\theta)\right)$ satisfies $\left(v_{01}, \gamma_{\epsilon}(\theta)\right) \in A_{\lambda}$. Let $\eta(t)=$ $\eta\left(t,\left(v_{01}, \gamma_{\epsilon}(\theta)\right)\right)$ be the maximal solution of (4.10) and $\left[0, \ell_{\theta}\right]$ be its maximal interval of existence satisfying $\eta(t) \in A_{\lambda}$ for all $t \in\left[0, \ell_{\theta}\right]$. Then for $\epsilon \in\left(0, \epsilon_{0}\right)$ small and $\lambda$ large we have

(i) $\left\|\frac{d \eta}{d t}\right\|_{T_{\eta(t)}\left(\Sigma_{1, \lambda} \times \Sigma_{2, \lambda}\right)} \leq 1$ for all $t \in\left[0, \ell_{\theta}\right]$.

(ii) $\frac{d}{d t} J_{\lambda}(\eta(t)) \leq 0$ for all $t \in\left[0, \ell_{\theta}\right]$.

(iii) $J_{\lambda}(\eta(t)) \leq c_{\text {min }}^{1, D}+c_{k}^{2, D}+\epsilon$ for all $t \in\left[0, \ell_{\theta}\right]$.

(iv) $\left(J_{1, \lambda}+J_{2, \lambda}\right)(\eta(t))<\left(1-r_{\lambda}\right)^{-\frac{2(p+1)}{p-1}}\left(c_{\text {min }}^{1, \lambda}+c_{k}^{2, \lambda}+\epsilon_{0}\right)$ for all $t \in\left[0, \ell_{\theta}\right]$.

(v) If $\eta(t)=\eta\left(t,\left(v_{01}, \gamma_{\epsilon}(\theta)\right)\right)$ satisfies $\eta\left(t_{0}\right) \in \partial A_{\lambda}$ for some $t_{0} \in\left[0, \ell_{\theta}\right]$, then

$$
\begin{aligned}
& \eta(t) \equiv\left(v_{01}, \gamma_{\epsilon}(\theta)\right) \quad \text { for all } t \in\left[0, \ell_{\theta}\right], \\
& J_{\lambda}\left(v_{01}, \gamma_{\epsilon}(\theta)\right) \leq c_{\text {min }}^{1, D}+c_{k}^{2, D}-\frac{2}{3} \epsilon_{0} .
\end{aligned}
$$

(vi) $\ell_{\theta}=\infty$ for all $\left(v_{01}, \gamma_{\epsilon}(\theta)\right) \in A_{\lambda}$. Moreover $\left(v_{01}, \gamma_{\epsilon}(\theta)\right) \in$ int $A_{\lambda}$ implies $\eta(t) \in$ int $A_{\lambda}$ for all $t \geq 0$ and $\left(v_{01}, \gamma_{\epsilon}(\theta)\right) \notin$ int $A_{\lambda}$ implies $\eta(t) \equiv$ $\left(v_{01}, \gamma_{\epsilon}(\theta)\right)$ for all $t \geq 0$.

(vii) If $\eta(t)=\eta\left(t,\left(v_{01}, \gamma_{\epsilon}(\theta)\right)\right)$ satisfies

$$
J_{\lambda}(\eta(t)) \geq c_{\min }^{1, D}+c_{k}^{2, D}-\frac{1}{3} \epsilon_{0},
$$

then $\eta(t) \in A_{\lambda}$ and $\frac{d}{d t} J_{\lambda}(\eta(t)) \leq-\mu_{\lambda}$. 
Proof. (i)-(ii) follow from the definition of $\widetilde{X}(v)$.

(iii) Since $J_{\lambda}\left(v_{01}, \gamma_{\epsilon}(\theta)\right)=J_{1, \lambda}\left(v_{01}\right)+J_{2, \lambda}\left(\gamma_{\epsilon}(\theta)\right) \leq c_{\text {min }}^{1, D}+c_{k}^{2, D}+\epsilon$, (iii) follows from (ii).

(iv) By (i) of Lemma 2.14 it follows from (iii) that

$$
\begin{aligned}
\left(J_{1, \lambda}+J_{2, \lambda}\right)(\eta(t)) & \leq\left(1-r_{\lambda}\right)^{-\frac{2(p+1)}{p-1}}\left(c_{\text {min }}^{1, D}+c_{k}^{2, D}+\epsilon\right) \\
& \leq\left(1-r_{\lambda}\right)^{-\frac{2(p+1)}{p-1}}\left(c_{\text {min }}^{1, \lambda}+c_{k}^{2, \lambda}+\epsilon_{0}\right) .
\end{aligned}
$$

Thus for sufficiently large $\lambda$, we have (iv).

(v) By (iv) and Remark 4.6, $\eta(t)=\left(\eta_{1}(t), \eta_{2}(t)\right)$ never touches a boundary $\left\{\left(v_{1}, v_{2}\right) ; J_{1, \lambda}\left(v_{1}\right)+J_{2, \lambda}\left(v_{2}\right)=\left(1-r_{\lambda}\right)^{-\frac{2(p+1)}{p-1}}\left(c_{m i n}^{1, \lambda}+c_{k}^{2, \lambda}+\epsilon_{0}\right)\right\} \cup\left\{\left(v_{1}, v_{2}\right) ; J_{1, \lambda}\left(v_{1}\right)=\right.$ $\left.c_{\text {min }}^{1, D}-\frac{1}{2} \delta_{0}\right\}$. We also remark that $\eta(t)$ never touches a boundary $\left\{\left(v_{1}, v_{2}\right) ; J_{1, \lambda}\left(v_{1}\right)\right.$ $=\beta$ by Lemma 4.5(iii) and (4.10).

Thus $\eta(t) \in \partial A_{\lambda}$ implies

$$
\left(J_{1, \lambda}+J_{2, \lambda}\right)(\eta(t))=\left(1+r_{\lambda}\right)^{-\frac{2(p+1)}{p-1}}\left(c_{\text {min }}^{1, \lambda}+c_{k}^{2, \lambda}-\epsilon_{0}\right) .
$$

We remark that (4.15) implies

$$
J_{\lambda}(\eta(t)) \leq c_{\text {min }}^{1, \lambda}+c_{k}^{2, \lambda}-\epsilon_{0} \leq c_{\text {min }}^{1, D}+c_{k}^{2, D}-\frac{2}{3} \epsilon_{0} .
$$

Since $\widetilde{X}(\eta(t))=0$ follows from (4.16), we have (v) from the uniqueness of solutions of (4.10). (vi)-(vii) also follow from the above arguments.

Proof of Theorem 1.1. We choose $\epsilon \in\left(0, \epsilon_{0}\right)$ and let $L_{\lambda}=\frac{2 \epsilon_{0}}{\mu_{\lambda}}>0$. We consider $\widetilde{\gamma}(\theta)=\eta\left(L_{\lambda},\left(v_{01}, \gamma_{\epsilon}(\theta)\right)\right): S_{+}^{n(k)+1} \rightarrow \Sigma_{1, \lambda} \times \Sigma_{2, \lambda}$. Then we can easily see $\widetilde{\gamma} \in \Lambda_{k}^{\lambda}$ and

$$
\sup _{\theta \in S_{+}^{n(k)+1}} J_{\lambda}(\widetilde{\gamma}(\theta)) \leq c_{\min }^{1, D}+c_{k}^{2, D}-\frac{1}{3} \epsilon_{0} .
$$

In fact, if $\left(v_{01}, \gamma_{\epsilon}(\theta)\right) \notin A_{\lambda}$, then by Lemma 4.8 we have

$$
J_{\lambda}(\widetilde{\gamma}(\theta)) \leq J_{\lambda}\left(v_{01}, \gamma_{\epsilon}(\theta)\right) \leq c_{\min }^{1, D}+c_{k}^{2, D}-\frac{2}{3} \epsilon_{0} .
$$

So we consider the case where $\left(v_{01}, \gamma_{\epsilon}(\theta)\right) \in A_{\lambda}$. If $J_{\lambda}\left(\eta\left(L_{\lambda},\left(v_{01}, \gamma_{\epsilon}(\theta)\right)\right)\right) \leq c_{\text {min }}^{1, D}+$ $c_{k}^{2, D}-\frac{1}{3} \epsilon_{0}$, then (4.17) clearly holds. On the other hand, if $J_{\lambda}\left(\eta\left(L_{\lambda},\left(v_{01}, \gamma_{\epsilon}(\theta)\right)\right)\right) \geq$ $c_{\text {min }}^{1, D}+c_{k}^{2, D}-\frac{1}{3} \epsilon_{0}$, then by (vii) of Lemma 4.9,

$$
\begin{aligned}
J_{\lambda}\left(\eta\left(L_{\lambda},\left(v_{01}, \gamma_{\epsilon}(\theta)\right)\right)\right) & =J_{\lambda}\left(v_{01}, \gamma_{\epsilon}(\theta)\right)+\int_{0}^{L_{\lambda}} \frac{d}{d t} J_{\lambda}(\eta(t)) d t \\
& \leq c_{\min }^{1, D}+c_{k}^{2, D}+\epsilon-\mu_{\lambda} L_{\lambda} \\
& \leq c_{\min }^{1, D}+c_{k}^{2, D}-\epsilon_{0},
\end{aligned}
$$

which is a contradiction.

Now we remark that (4.17) implies

$$
c_{k}^{\lambda}=\inf _{\gamma \in \Lambda_{k}^{\lambda}} \sup _{\theta \in S_{+}^{n(k)+1}} J_{\lambda}(\gamma(\theta)) \leq \sup _{\theta \in S_{+}^{n(k)+1}} J_{\lambda}(\widetilde{\gamma}(\theta)) \leq c_{m i n}^{1, D}+c_{k}^{2, D}-\frac{1}{3} \epsilon_{0} .
$$

However, by Proposition 3.7, we have $c_{k}^{\lambda} \rightarrow c_{\text {min }}^{1, D}+c_{k}^{2, D}$. This is a contradiction and $J_{\lambda}(v)$ has a critical point in $A_{\lambda}$. Thus Theorem 1.1 is proved. 


\section{ONE DIMENSIONAL CASE}

In this section we consider 1 dimensional case:

$\left(P_{\lambda}\right) \quad-u^{\prime \prime}+\left(\lambda^{2} a(x)+1\right) u=|u|^{p-1} u \quad$ in $\mathbf{R}, \quad u(x) \in H^{1}(\mathbf{R})$.

For $a(x)$, we assume

(a1) $a(x) \in C(\mathbf{R}, \mathbf{R}), a(x) \geq 0$ for all $x \in \mathbf{R}$ and the potential well consists of 2 intervals, that is, int $a^{-1}(0)=\Omega_{1} \cup \Omega_{2}, \Omega_{1}=\left(a_{1}, b_{1}\right)$ and $\Omega_{2}=\left(a_{2}, b_{2}\right)$ $\left(a_{1}<b_{1}<a_{2}<b_{2}\right)$.

(a2) $0<\liminf _{|x| \rightarrow \infty} a(x) \leq \sup _{x \in \mathbf{R}} a(x)<\infty$.

(a) Limit equations. For $-\infty<a<b<\infty$, we consider the following equation:

$$
-u^{\prime \prime}+u=|u|^{p-1} u \quad \text { in }(a, b), \quad u(a)=u(b)=0 .
$$

For any $n \in \mathbf{N}$, there exists a unique solution $\widetilde{w}_{n}(a, b ; x)$ of (5.1) which has the following property (cf. 9]):

$$
\widetilde{w}_{n}(a, b ; x) \text { possesses exactly } n-1 \text { zeros in }(a, b) \text { and } \widetilde{w}_{n}^{\prime}(a, b ; a)>0 .
$$

Moreover we have

$$
\begin{aligned}
& 1^{\circ} \widetilde{w}_{n}(a, b ; x)=\widetilde{w}_{n}(0, b-a ; x-a) . \\
& 2^{\circ} \widetilde{w}_{n}^{\prime}\left(0, \ell_{1} ; 0\right)>\widetilde{w}_{n}^{\prime}\left(0, \ell_{2} ; 0\right) \text { for } \ell_{2}>\ell_{1}>0 . \\
& 3^{\circ} \widetilde{w}_{n}(0, \ell ; x)=(-1)^{k-1} \widetilde{w}_{1}\left(0, \frac{\ell}{n} ; x-(k-1) \frac{\ell}{n}\right) \text { for } x \in\left((k-1) \frac{\ell}{n}, k \frac{\ell}{n}\right), \ell> \\
& \quad 0, k=1, \cdots, n .
\end{aligned}
$$

We remark that the set of all solutions of (5.1) can be described as $\left\{ \pm \widetilde{w}_{n}(a, b ; x) ; n \in\right.$ $\mathbf{N}\} \cup\{0\}$. In what follows, we define

$$
w_{n}(a, b ; x)= \begin{cases}\widetilde{w}_{n}(a, b ; x) & \text { in }(a, b), \\ 0 & \text { otherwise. }\end{cases}
$$

(b) The proof of Theorem 1.2. We have two limit problems for $\left(P_{\lambda}\right)$ : for $i=1,2$

$$
-u^{\prime \prime}+u=|u|^{p-1} u \quad \text { in }\left(a_{i}, b_{i}\right), \quad u\left(a_{i}\right)=u\left(b_{i}\right)=0 .
$$

In order to prove Theorem 1.2, for any $n_{1}, n_{2} \in \mathbf{N}, s_{1}, s_{2} \in\{-1,+1\}$ we will construct solutions of $\left(P_{\lambda}\right)$ which converge to $s_{i} w_{n_{i}}\left(a_{i}, b_{i} ; x\right)$ in $\left(a_{i}, b_{i}\right)$ and to 0 elsewhere. We use the broken-geodesic method which is used in [15. See also [19] and [26] for related arguments. We consider the case $n_{1}, n_{2} \geq 3$. We can deal with the other cases in a similar way.

We set $t_{1}^{0}=a_{1}+\frac{b_{1}-a_{1}}{n_{1}}, t_{2}^{0}=b_{1}-\frac{b_{1}-a_{1}}{n_{1}}, t_{3}^{0}=a_{2}+\frac{b_{2}-a_{2}}{n_{2}}, t_{4}^{0}=b_{2}-\frac{b_{2}-a_{2}}{n_{2}}$, $\mathbf{t}^{0}=\left(t_{1}^{0}, t_{2}^{0}, t_{3}^{0}, t_{4}^{0}\right)$. For small $\epsilon \in\left(0, \min \left\{\frac{b_{1}-a_{1}}{n_{1}}, \frac{b_{2}-a_{2}}{n_{2}}\right\}\right)$ we set

$$
U=\prod_{i=1}^{4}\left(t_{i}^{0}-\epsilon, t_{i}^{0}+\epsilon\right) .
$$

To find solutions of $\left(P_{\lambda}\right)$, first we fix $\mathbf{t}=\left(t_{1}, t_{2}, t_{3}, t_{4}\right) \in U, t_{0}=-\infty, t_{5}=\infty$ and we solve $\left(P_{\lambda}\right)$ in the intervals $\left(t_{i}, t_{i+1}\right)$ for $i=0,1, \cdots, 4$ :

$\left(P_{\lambda, i}\right) \quad-u^{\prime \prime}+\left(\lambda^{2} a(x)+1\right) u=|u|^{p-1} u \quad$ in $\left(t_{i}, t_{i+1}\right), \quad u(x) \in H_{0}^{1}\left(t_{i}, t_{i+1}\right)$.

Remark 5.1. We remark that $\left(P_{\lambda, 1}\right)$ and $\left(P_{\lambda, 3}\right)$ are independent of $\lambda$. Thus $\left(P_{\lambda, 1}\right)$ $\left(\left(P_{\lambda, 3}\right)\right.$ resp. $)$ has a unique solution $w_{n_{1}-2}\left(t_{1}, t_{2} ; x\right)\left(w_{n_{2}-2}\left(t_{3}, t_{4} ; x\right)\right.$ resp. $)$, which has $n_{1}-3\left(n_{2}-3\right.$ resp. $)$ zeros and $w_{n_{1}-2}^{\prime}\left(t_{1}, t_{2} ; t_{1}\right)>0\left(w_{n_{2}-2}^{\prime}\left(t_{3}, t_{4} ; t_{3}\right)>0\right.$ resp. $)$. 
The following lemma deals with $\left(P_{\lambda, i}\right)(i=0,2,4)$, which can be shown just as in [15].

Lemma 5.2. There exists $\delta_{0}>0$ such that for any $\delta \in\left(0, \delta_{0}\right)$, there exists $\Lambda_{0}=$ $\Lambda_{0}(\delta)>0$ such that for $\lambda>\Lambda_{0}$ and $\left(t_{1}, t_{2}, t_{3}, t_{4}\right) \in U$,

(i) $\left(P_{\lambda, 0}\right)$ has a unique solution $u_{0, \lambda}\left(t_{1} ; x\right)$ which satisfies

$$
\left\|u_{0, \lambda}\left(t_{1} ; x\right)-w_{1}\left(a_{1}, t_{1} ; x\right)\right\|_{L^{\infty}\left(-\infty, t_{1}\right)}<\delta .
$$

(ii) $\left(P_{\lambda, 2}\right)$ has a unique solution $u_{2, \lambda}\left(t_{2}, t_{3} ; x\right)$ which satisfies

$$
\left\|u_{2, \lambda}\left(t_{2}, t_{3} ; x\right)-w_{1}\left(t_{2}, b_{1} ; x\right)-w_{1}\left(a_{2}, t_{3} ; x\right)\right\|_{L^{\infty}\left(t_{2}, t_{3}\right)}<\delta .
$$

Similarly, $\left(P_{\lambda, 2}\right)$ has a unique solution $\widehat{u}_{2, \lambda}\left(t_{2}, t_{3} ; x\right)$ which satisfies

$$
\left\|\widehat{u}_{2, \lambda}\left(t_{2}, t_{3} ; x\right)-w_{1}\left(t_{2}, b_{1} ; x\right)+w_{1}\left(a_{2}, t_{3} ; x\right)\right\|_{L^{\infty}\left(t_{2}, t_{3}\right)}<\delta .
$$

(iii) $\left(P_{\lambda, 4}\right)$ has a unique solution $u_{4, \lambda}\left(t_{4} ; x\right)$ which satisfies

$$
\left\|u_{4, \lambda}\left(t_{4} ; x\right)-w_{1}\left(t_{4}, b_{2} ; x\right)\right\|_{L^{\infty}\left(t_{4}, \infty\right)}<\delta .
$$

Remark 5.3. From Lemma $5.2, u_{0, \lambda}\left(t_{1} ; x\right)$ satisfies

$$
\left\|u_{0, \lambda}\left(t_{1} ; x\right)-w_{1}\left(a_{1}, t_{1} ; x\right)\right\|_{L^{\infty}\left(-\infty, t_{1}\right)} \rightarrow 0 \quad \text { as } \lambda \rightarrow \infty .
$$

Since $\left(P_{\lambda, 0}\right)$ is independent of $\lambda$ in $\left[a_{1}, t_{1}\right]$, (5.2) implies

$$
\left\|u_{0, \lambda}\left(t_{1} ; x\right)-w_{1}\left(a_{1}, t_{1} ; x\right)\right\|_{C^{2}\left(\left[a_{1}, t_{1}\right]\right)} \rightarrow 0 \quad \text { as } \lambda \rightarrow \infty .
$$

By uniqueness of $u_{0, \lambda}\left(t_{1} ; x\right)$, we find $u_{0, \lambda}\left(t_{1} ; t_{1}\right)$ and $u_{0, \lambda}^{\prime}\left(t_{1} ; t_{1}\right)$ are continuous with respect to $t_{1} \in\left(t_{1}^{0}-\epsilon, t_{1}^{0}+\epsilon\right)$. Similar results hold for $u_{2, \lambda}\left(t_{2}, t_{3} ; x\right), \widehat{u}_{2, \lambda}\left(t_{2}, t_{3} ; x\right)$ and $u_{4, \lambda}\left(t_{4} ; x\right)$.

End of the proof of Theorem 1.2. Let us outline the proof for the case $n_{1}, n_{2} \geq$ 3. We can deal with the other cases in a similar way. We define functions $u_{\lambda}(\mathbf{t} ; x), \widehat{u}_{\lambda}(\mathbf{t} ; x)$ for $\mathbf{t}=\left(t_{1}, \cdots, t_{4}\right) \in U$ by

$$
\begin{aligned}
u_{\lambda}(\mathbf{t} ; x)= & u_{0, \lambda}\left(t_{1} ; x\right)-w_{n_{1}-2}\left(t_{1}, t_{2} ; x\right)+(-1)^{n_{1}-1} u_{2, \lambda}\left(t_{2}, t_{3} ; x\right) \\
& +(-1)^{n_{1}} w_{n_{2}-2}\left(t_{3}, t_{4} ; x\right)+(-1)^{n_{1}+n_{2}} u_{4, \lambda}\left(t_{4} ; x\right)
\end{aligned}
$$

or

$$
\begin{aligned}
\widehat{u}_{\lambda}(\mathbf{t} ; x)= & u_{0, \lambda}\left(t_{1} ; x\right)-w_{n_{1}-2}\left(t_{1}, t_{2} ; x\right)+(-1)^{n_{1}-1} \widehat{u}_{2, \lambda}\left(t_{2}, t_{3} ; x\right) \\
& +(-1)^{n_{1}-1} w_{n_{2}-2}\left(t_{3}, t_{4} ; x\right)+(-1)^{n_{1}+n_{2}-1} u_{4, \lambda}\left(t_{4} ; x\right) .
\end{aligned}
$$

We deal with $u_{\lambda}(\mathbf{t} ; x)$. We can deal with $\widehat{u}_{\lambda}(\mathbf{t} ; x)$ in a similar way. We observe that $u_{\lambda}(\mathbf{t} ; x)$ is a solution of $\left(P_{\lambda}\right)$ if and only if $u_{\lambda}(\mathbf{t} ; x)$ is of class $C^{1}$ with respect to $x$. We define a function $F_{\lambda}(\mathbf{t}) \in C\left(U, \mathbf{R}^{4}\right)$ by

$$
F_{\lambda}(\mathbf{t})=\left[\begin{array}{c}
F_{1, \lambda}(\mathbf{t}) \\
F_{2, \lambda}(\mathbf{t}) \\
F_{3, \lambda}(\mathbf{t}) \\
F_{4, \lambda}(\mathbf{t})
\end{array}\right]=\left[\begin{array}{c}
w_{n_{1}-2}^{\prime}\left(t_{1}, t_{2} ; t_{1}\right)+u_{0, \lambda}^{\prime}\left(t_{1} ; t_{1}\right) \\
u_{2, \lambda}^{\prime}\left(t_{2}, t_{3} ; t_{2}\right)+(-1)^{n_{1}-1} w_{n_{1}-2}^{\prime}\left(t_{1}, t_{2} ; t_{2}\right) \\
w_{n_{2}-2}^{\prime}\left(t_{3}, t_{4} ; t_{3}\right)+u_{2, \lambda}^{\prime}\left(t_{2}, t_{3} ; t_{3}\right) \\
u_{2, \lambda}^{\prime}\left(t_{4} ; t_{4}\right)+(-1)^{n_{2}-1} w_{n_{2}-2}^{\prime}\left(t_{3}, t_{4} ; t_{4}\right)
\end{array}\right]: U \rightarrow \mathbf{R}^{4} .
$$

Then $u_{\lambda}(\mathbf{t} ; x)$ is of class $C^{1}$ if and only if $F_{\lambda}(\mathbf{t})=0$. We will show $\operatorname{deg}\left(F_{\lambda}, U, 0\right)=1$. We remark that $\partial U=\bigcup_{i=1}^{4}\left(\left\{t_{i}=t_{i}^{0}-\epsilon\right\} \cup\left\{t_{i}=t_{i}^{0}+\epsilon\right\}\right)$. For $i=1,2,3$, 4, we have

$$
F_{i, \lambda}(\mathbf{t})<0 \quad \text { if } \quad t_{i}=t_{i}^{0}-\epsilon, \quad F_{i, \lambda}(\mathbf{t})>0 \quad \text { if } \quad t_{i}=t_{i}^{0}+\epsilon
$$


for large $\lambda$. In fact, when $t_{1}=t_{1}^{0}-\epsilon$, by the properties of $w_{n}(a, b ; x)$, we find

$$
\begin{aligned}
F_{1, \lambda}(\mathbf{t}) & =w_{n_{1}-2}^{\prime}\left(t_{1}^{0}-\epsilon, t_{2} ; t_{1}^{0}-\epsilon\right)+u_{0, \lambda}^{\prime}\left(t_{1}^{0}-\epsilon ; t_{1}^{0}-\epsilon\right) \\
& \leq w_{n_{1}-2}^{\prime}\left(t_{1}^{0}-\epsilon, t_{2}^{0}-\epsilon ; t_{1}^{0}-\epsilon\right)+u_{0, \lambda}^{\prime}\left(t_{1}^{0}-\epsilon ; t_{1}^{0}-\epsilon\right) \\
& =w_{1}^{\prime}\left(a_{1}, t_{1}^{0} ; a_{1}\right)+u_{0, \lambda}^{\prime}\left(t_{1}^{0}-\epsilon ; t_{1}^{0}-\epsilon\right) .
\end{aligned}
$$

By Remark 5.3, we observe

$$
w_{1}^{\prime}\left(a_{1}, t_{1}^{0} ; a_{1}\right)+u_{0, \lambda}^{\prime}\left(t_{1}^{0}-\epsilon ; t_{1}^{0}-\epsilon\right) \rightarrow w_{1}^{\prime}\left(a_{1}, t_{1}^{0} ; a_{1}\right)+w_{1}^{\prime}\left(a_{1}, t_{1}^{0}-\epsilon ; t_{1}^{0}-\epsilon\right)<0 \text { as } \lambda \rightarrow \infty .
$$

We can show the other properties in (5.3) in a similar way. By (5.3) $\operatorname{deg}\left(F_{\lambda}, U, 0\right)$ is well-defined for large $\lambda$ and $\operatorname{deg}\left(F_{\lambda}, U, 0\right)=1$. Therefore there exists $\mathbf{t}_{\lambda}^{*} \in U$ such that $u_{\lambda}\left(\mathbf{t}_{\lambda}^{*} ; x\right)$ is a solution of $\left(P_{\lambda}\right)$. Since $\epsilon>0$ is arbitrary, we can show $\mathbf{t}_{\lambda}^{*} \rightarrow \mathbf{t}^{0}$ as $\lambda \rightarrow \infty$; that is, $u_{\lambda}\left(\mathbf{t}_{\lambda}^{*} ; x\right)$ converges to $w_{n_{1}}\left(a_{1}, b_{1} ; x\right)$ in $\left(a_{1}, b_{1}\right)$, to $(-1)^{n_{1}-1} w_{n_{2}}\left(a_{2}, b_{2} ; x\right)$ in $\left(a_{2}, b_{2}\right)$ and to 0 elsewhere.

\section{Proofs of Theorems 1.3 and 1.5}

In this section we give proofs of Theorem 1.3 and Theorem 1.5. Since the exponent $p$ plays an important role, we write dependence of $J_{\lambda}, J_{i, D}$ on $p$ explicitly in this section and use the notation

$$
\begin{aligned}
J_{\lambda}\left(p ; v_{1}, v_{2}\right) & =J_{\lambda}\left(v_{1}, v_{2}\right) \quad \text { for }\left(v_{1}, v_{2}\right) \in \Sigma_{1, \lambda} \times \Sigma_{2, \lambda}, \\
J_{i, D}\left(p ; v_{i}\right) & =\left(\frac{1}{2}-\frac{1}{p+1}\right)\left(\frac{1}{\left\|v_{i}\right\|_{L^{p+1}\left(\Omega_{i}\right)}}\right)^{\frac{2(p+1)}{p-1}} \text { for } v_{i} \in \Sigma_{i, D} .
\end{aligned}
$$

We define

$$
c_{p}^{\lambda}=\inf _{\left(v_{1}, v_{2}\right) \in \Sigma_{1, \lambda} \times \Sigma_{2, \lambda}} J_{\lambda}\left(p ; v_{1}, v_{2}\right), \quad c_{p}\left(\Omega_{i}\right)=\inf _{v_{i} \in \Sigma_{i, D}} J_{i, D}\left(p ; v_{i}\right) .
$$

Since $J_{\lambda}\left(p ; v_{1}, v_{2}\right), J_{i, D}\left(p ; v_{i}\right)$ satisfy the Palais-Smale condition, $c_{p}^{\lambda}$ and $c_{p}\left(\Omega_{i}\right)$ are critical values of $J_{\lambda}\left(p ; v_{1}, v_{2}\right)$ and $J_{i, D}\left(p ; v_{i}\right)$. First we observe

Lemma 6.1. $\quad$ (i) $c_{p}^{\lambda}<c_{p}\left(\Omega_{1}\right)+c_{p}\left(\Omega_{2}\right)$ for all $\lambda \geq 1$.

(ii) $c_{p}^{\lambda} \rightarrow c_{p}\left(\Omega_{1}\right)+c_{p}\left(\Omega_{2}\right)$ as $\lambda \rightarrow \infty$.

Proof. Let $\left(v_{1, \lambda}, v_{2, \lambda}\right) \in \Sigma_{1, \lambda} \times \Sigma_{2, \lambda}$ be a minimizer of $J_{\lambda}\left(p ; v_{1}, v_{2}\right)$, that is, $J_{\lambda}\left(p ; v_{1, \lambda}, v_{2, \lambda}\right)=c_{p}^{\lambda}$. (i) Since $\Sigma_{1, D} \times \Sigma_{2, D} \subset \Sigma_{1, \lambda} \times \Sigma_{2, \lambda}$, we have

$$
\begin{aligned}
c_{p}^{\lambda} & =\inf _{\left(v_{1}, v_{2}\right) \in \Sigma_{1, \lambda} \times \Sigma_{2, \lambda}} J_{\lambda}\left(p ; v_{1}, v_{2}\right) \leq \inf _{\left(v_{1}, v_{2}\right) \in \Sigma_{1, D} \times \Sigma_{2, D}} J_{\lambda}\left(p ; v_{1}, v_{2}\right) \\
& =\inf _{\left(v_{1}, v_{2}\right) \in \Sigma_{1, D} \times \Sigma_{2, D}}\left(J_{1, D}\left(p ; v_{1}\right)+J_{2, D}\left(p ; v_{2}\right)\right)=c_{p}\left(\Omega_{1}\right)+c_{p}\left(\Omega_{2}\right) .
\end{aligned}
$$

Since $\left(v_{1, \lambda}, v_{2, \lambda}\right)$ corresponds to a positive solution $u(x)$ of $-\Delta u+\left(\lambda^{2} a(x)+1\right) u=$ $g(x, u)$ in $\mathbf{R}^{N}$, we have $v_{i, \lambda} \in \Sigma_{i, \lambda} \backslash \Sigma_{i, D}$ and we have strict inequality.

(ii) By Proposition 2.17 there exist a sequence $\lambda_{n} \rightarrow \infty$ and critical points $v_{i} \in \Sigma_{i, D}$ of $J_{i, D}(i=1,2)$ such that

$$
\left(v_{1, \lambda_{n}}, v_{2, \lambda_{n}}\right) \rightarrow\left(v_{1}, v_{2}\right) \text { strongly in } H^{1}\left(\Omega_{1}^{\prime}\right) \oplus H^{1}\left(\Omega_{2}^{\prime}\right)
$$

and

$$
J_{\lambda_{n}}\left(p ; v_{1, \lambda_{n}}, v_{2, \lambda_{n}}\right) \rightarrow J_{1, D}\left(p ; v_{1}\right)+J_{2, D}\left(p ; v_{2}\right) \geq c_{p}\left(\Omega_{1}\right)+c_{p}\left(\Omega_{2}\right) .
$$

Therefore by (i), $c_{p}^{\lambda_{n}} \rightarrow c_{p}\left(\Omega_{1}\right)+c_{p}\left(\Omega_{2}\right)$. Since the limit does not depend on the subsequence, conclusion (ii) holds. 
To study the case $p \sim \frac{N+2}{N-2}$, we introduce the notation

$$
c_{p}(Q)=\inf _{u \in H_{0}^{1}(Q),\|u\|_{H^{1}(Q)}=1}\left(\frac{1}{2}-\frac{1}{p+1}\right)\left(\frac{1}{\|u\|_{L^{p+1}(Q)}}\right)^{\frac{2(p+1)}{p-1}} \quad \text { for } Q \subset \mathbf{R}^{N} .
$$

$c_{p}(Q)$ has the following properties:

Lemma 6.2. (0) $Q \subset Q^{\prime}$ implies $c_{p}\left(Q^{\prime}\right) \leq c_{p}(Q)$.

(i) $c_{\frac{N+2}{N-2}} \equiv c_{\frac{N+2}{N-2}}(Q)$ does not depend on $Q$.

(ii) $c_{\frac{N+2}{N-2}}=c_{\frac{N+2}{N-2}}(Q)$ is never achieved in a proper subset of $\mathbf{R}^{N}$.

(iii) For any $Q \subset \mathbf{R}^{N}, c_{p}(Q) \rightarrow c_{\frac{N+2}{N-2}}$ as $p \rightarrow \frac{N+2}{N-2}-0$.

Proof. Introducing

$$
d_{p}(Q)=\inf _{u \in H_{0}^{1}(Q),\|u\|_{H^{1}(Q)}=1} \frac{1}{\|u\|_{L^{p+1}(Q)}^{2}} \quad \text { for } Q \subset \mathbf{R}^{N},
$$

(0)-(iii) are equivalent to

(0) $Q \subset Q^{\prime}$ implies $d_{p}\left(Q^{\prime}\right) \leq d_{p}(Q)$.

(i) $d_{\frac{N+2}{N-2}} \equiv d_{\frac{N+2}{N-2}}(Q)$ does not depend on $Q$.

(ii) $d_{\frac{N+2}{N-2}}=d_{\frac{N+2}{N-2}}(Q)$ is never achieved in a proper subset of $\mathbf{R}^{N}$.

(iii) For any $Q \subset \mathbf{R}^{N}, d_{p}(Q) \rightarrow d_{\frac{N+2}{N-2}}$ as $p \rightarrow \frac{N+2}{N-2}-0$,

which can be derived as in [7] (see page 81 and Lemma 4.1).

For $r>0$ we set

$$
\Omega_{i}^{+}=\left\{x \in \mathbf{R}^{N} ; \operatorname{dist}\left(x, \Omega_{i}\right)<r\right\}, \quad \Omega_{i}^{-}=\left\{x \in \Omega_{i} ; \operatorname{dist}\left(x, \partial \Omega_{i}\right)>r\right\} .
$$

We choose $r>0$ small enough so that

(i) The inclusions $\Omega_{i}^{-} \subset \Omega_{i}$ and $\Omega_{i} \subset \Omega_{i}^{+}$are homotopy equivalent.

(ii) $B_{r}(x) \subset \Omega_{i}$ for all $x \in \Omega_{i}^{-}$.

By the choice of $r>0$, we have

Corollary 6.3. $\quad$ (i) $c_{p}^{\lambda}<c_{p}\left(\Omega_{1}\right)+c_{p}\left(\Omega_{2}\right)<2 c_{p}\left(B_{r}\right)$.

(ii) There exists $p_{1} \in\left(1, \frac{N+2}{N-2}\right)$ such that for any $p \in\left(p_{1}, \frac{N+2}{N-2}\right)$, there exists $\Lambda_{1}(p)>0$ such that

$$
c_{p}\left(B_{r}\right)<c_{p}^{\lambda} \text { for all } \lambda \geq \Lambda_{1}(p) .
$$

Proof. (i) follows from Lemma 6.1 (i) and Lemma 6.2 (0). (ii) By Lemma 6.2 (iii), we have $c_{p}\left(\Omega_{1}\right)+c_{p}\left(\Omega_{2}\right) \rightarrow 2 c_{\frac{N+2}{N-2}}, c_{p}\left(B_{r}\right) \rightarrow c_{\frac{N+2}{N-2}}$. Thus (ii) follows from Lemma 6.1 (ii).

For $v \in \Sigma_{i, \lambda}$ we set

$$
\beta_{i}(p ; v)=\frac{\int_{\Omega_{i}^{\prime}}|v|^{p+1} x d x}{\int_{\Omega_{i}^{\prime}}|v|^{p+1} d x}: \Sigma_{i, \lambda} \rightarrow \mathbf{R}^{N} .
$$

We have the following.

Lemma 6.4. There exists $\delta>0$ such that if $p \in\left(\frac{N+2}{N-2}-\delta, \frac{N+2}{N-2}\right)$ and $v \in \Sigma_{i, D}$ satisfies

then $\beta_{i}(p ; v) \in \Omega_{i}^{+}$.

$$
J_{i, D}(p ; v) \leq c_{\frac{N+2}{N-2}}+\delta
$$


We remark that

$$
\inf _{v \in \Sigma_{i, D}} J_{i, D}(p ; v)=c_{p}\left(\Omega_{i}\right) \rightarrow c_{\frac{N+2}{N-2}} \quad \text { as } \quad p \rightarrow \frac{N+2}{N-2}-0 .
$$

Thus $\left\{v \in \Sigma_{i, D} ; J_{i, D}(p ; v) \leq c_{\frac{N+2}{N-2}}+\delta\right\}$ is not empty for $p$ close to $\frac{N+2}{N-2}$.

Proof. We argue indirectly and assume that there exist $p_{n}, v_{n} \in \Sigma_{i, D}$ such that

$$
p_{n} \rightarrow \frac{N+2}{N-2}, \quad J_{i, D}\left(p_{n} ; v_{n}\right) \rightarrow c_{\frac{N+2}{N-2}}, \quad \beta_{i}\left(p_{n} ; v_{n}\right) \notin \Omega_{i}^{+} .
$$

By Hölder's inequality,

$$
\begin{aligned}
& J_{i, D}\left(\frac{N+2}{N-2} ; v_{n}\right) \\
= & \frac{1}{N} \frac{1}{\left\|v_{n}\right\|_{L^{\frac{2 N}{N-2}}\left(\Omega_{i}\right)}^{N}} \leq \frac{1}{N}\left|\Omega_{i}\right|^{\frac{\frac{N+2}{N-2}-p_{n}}{p_{n}+1}} \frac{1}{\left\|v_{n}\right\|_{L^{p_{n}+1}\left(\Omega_{i}\right)}^{N}} \\
= & \frac{1}{N}\left|\Omega_{i}\right|^{-\frac{\frac{N+2}{N-2}-p_{n}}{p_{n}+1}}\left(\frac{1}{2}-\frac{1}{p_{n}+1}\right)^{-\frac{p_{n}-1}{p_{n}+1} \frac{N}{2}} J_{i, D}\left(p_{n} ; v_{n}\right)^{\frac{p_{n}-1}{p_{n}+1} \frac{N}{2}} \\
\rightarrow & c_{\frac{N+2}{N-2}} .
\end{aligned}
$$

Since $J_{i, D}\left(\frac{N+2}{N-2} ; v\right) \geq c_{\frac{N+2}{N-2}}$ for all $v \in \Sigma_{i, D}$, it follows from (6.2) that

$$
\frac{1}{\left\|v_{n}\right\|_{L^{\frac{2 N}{N-2}}}^{2}\left(\Omega_{i}\right)} \rightarrow d_{\frac{N+2}{N-2}},
$$

where $d_{\frac{N+2}{N-2}}$ is defined in the proof of Lemma 6.2. From the well-known compactness result (cf. Struwe [24] and Lions [18), it follows that there exist $r_{n} \rightarrow 0$, $\left(x_{n}\right)_{n=1}^{\infty} \subset \Omega_{i}$ and a function $w_{0}(x) \in \mathcal{D}^{1,2}\left(\mathbf{R}^{N}\right)$ satisfying $\left\|\nabla w_{0}\right\|_{L^{2}\left(\mathbf{R}^{N}\right)}=1$ and $\left\|w_{0}\right\|_{L^{\frac{2 N}{N-2}}\left(\mathbf{R}^{N}\right)}^{2}=d_{\frac{N+2}{N-2}}^{-1}$ such that $r_{n}^{\frac{N-2}{2}} v_{n}\left(r_{n} x+x_{n}\right) \rightarrow w_{0}(x)$ strongly in $\mathcal{D}^{1,2}\left(\mathbf{R}^{N}\right)$. Thus

$$
\beta_{i}\left(p_{n} ; v_{n}\right) \in \Omega_{i}^{+} \quad \text { for large } n .
$$

This is a contradiction to assumption (6.1).

A following proposition is a key of the proof of Theorem 1.3.

Proposition 6.5. There exists $p_{2} \in\left(1, \frac{N+2}{N-2}\right)$ such that for any $p \in\left(p_{2}, \frac{N+2}{N-2}\right)$, there exists $\Lambda_{2}(p) \geq 1$ such that $\left(\beta_{1}\left(p ; v_{1}\right), \beta_{2}\left(p ; v_{2}\right)\right) \in \Omega_{1}^{+} \times \Omega_{2}^{+}$for all $\lambda \geq \Lambda_{2}(p)$ and $\left(v_{1}, v_{2}\right) \in \Sigma_{1, \lambda} \times \Sigma_{2, \lambda}$ satisfying $J_{\lambda}\left(p ; v_{1}, v_{2}\right) \leq 2 c_{p}\left(B_{r}\right)$.

We remark by Corollary 6.3 (i) that the level set

$$
\begin{aligned}
& {\left[J_{\lambda}\left(p ; v_{1}, v_{2}\right) \leq 2 c_{p}\left(B_{r}\right)\right]_{\Sigma_{1, \lambda} \times \Sigma_{2, \lambda}} } \\
= & \left\{\left(v_{1}, v_{2}\right) \in \Sigma_{1, \lambda} \times \Sigma_{2, \lambda} ; J_{\lambda}\left(p ; v_{1}, v_{2}\right) \leq 2 c_{p}\left(B_{r}\right)\right\}
\end{aligned}
$$

is not empty.

Proof. We choose $p_{2} \in\left(p_{1}, \frac{N+2}{N-2}\right)$ such that

$$
2 c_{p}\left(B_{r}\right)-c_{p}\left(\Omega_{i}\right) \leq c_{\frac{N+2}{N-2}}+\delta \text { for } i=1,2 \text { and } p \in\left(p_{2}, \frac{N+2}{N-2}\right),
$$

where $\delta>0$ is given in Lemma 6.4 We will show the conclusion of Proposition 6.5 holds for $p_{2}$. 
Again we argue indirectly, and we assume that there exist $p \in\left(p_{2}, \frac{N+2}{N-2}\right), \lambda_{n} \rightarrow \infty$ and $\left(v_{1 n}, v_{2 n}\right) \in \Sigma_{1, \lambda_{n}} \times \Sigma_{2, \lambda_{n}}$ such that

$$
J_{\lambda_{n}}\left(p ; v_{1 n}, v_{2 n}\right) \leq 2 c_{p}\left(B_{r}\right), \quad\left(\beta_{1}\left(p ; v_{1 n}\right), \beta_{2}\left(p ; v_{2 n}\right)\right) \notin \Omega_{1}^{+} \times \Omega_{2}^{+} .
$$

By Proposition 2.15, $\left(v_{1 n}, v_{2 n}\right)$ satisfies $\left\|v_{i n}\right\|_{L^{p+1}\left(\Omega_{i}^{\prime}\right)} \geq \delta_{M}$, where $\delta_{M}>0$ is given in Proposition 2.15 with $M=2 c_{\frac{N+2}{N-2}}+1$. Extracting a subsequence if necessary, we may assume

$$
v_{i n} \rightarrow v_{i 0} \quad \text { strongly in } L^{p+1}\left(\Omega_{i}^{\prime}\right) \text { and weakly in } H^{1}\left(\Omega_{i}^{\prime}\right) .
$$

Thus we have $\left\|v_{i 0}\right\|_{L^{p+1}\left(\Omega_{i}^{\prime}\right)} \geq \delta_{M}$ and

$$
\left(\beta_{1}\left(p ; v_{10}\right), \beta_{2}\left(p ; v_{20}\right)\right) \notin \Omega_{1}^{+} \times \Omega_{2}^{+} .
$$

We also remark that for any compact set $D \subset \Omega_{i}^{\prime} \backslash \overline{\Omega_{i}}$

$$
\left\|v_{i n}\right\|_{L^{2}(D)}^{2} \leq \frac{1}{\lambda_{n}^{2} \inf _{x \in D} a(x)}\left\|v_{i n}\right\|_{\lambda_{n}, \Omega_{i}^{\prime}}^{2} \rightarrow 0
$$

In particular, we have $v_{i 0} \in H_{0}^{1}\left(\Omega_{i}\right)$. Thus we have $\frac{v_{i 0}}{\left\|v_{i 0}\right\|_{H^{1}\left(\Omega_{i}\right)}} \in \Sigma_{i, D}$, and it follows from $\left\|v_{i 0}\right\|_{H^{1}\left(\Omega_{i}\right)} \leq \liminf _{n \rightarrow \infty}\left\|v_{i n}\right\|_{H^{1}\left(\Omega_{i}^{\prime}\right)} \leq \liminf _{n \rightarrow \infty}\left\|v_{i n}\right\|_{\lambda_{n}, \Omega_{i}^{\prime}}=1$ that

$$
\begin{aligned}
J_{i, D}\left(\frac{v_{i 0}}{\left\|v_{i 0}\right\|_{H^{1}\left(\Omega_{i}\right)}}\right) & =\left(\frac{1}{2}-\frac{1}{p+1}\right)\left(\frac{\left\|v_{i 0}\right\|_{H^{1}\left(\Omega_{i}\right)}}{\left\|v_{i 0}\right\|_{L^{p+1}\left(\Omega_{i}\right)}}\right)^{\frac{2(p+1)}{p-1}} \\
& \leq\left(\frac{1}{2}-\frac{1}{p+1}\right)\left\|v_{i 0}\right\|_{L^{p^{p+1}\left(\Omega_{i}\right)}}^{-\frac{2(p+1)}{p-1}}
\end{aligned}
$$

Thus we have

$$
\begin{aligned}
& J_{1, D}\left(\frac{v_{10}}{\left\|v_{10}\right\|_{H^{1}\left(\Omega_{1}\right)}}\right)+J_{2, D}\left(\frac{v_{20}}{\left\|v_{20}\right\|_{H^{1}\left(\Omega_{2}\right)}}\right) \\
\leq & \left(\frac{1}{2}-\frac{1}{p+1}\right)\left(\left\|v_{10}\right\|_{L^{p+1}\left(\Omega_{1}^{\prime}\right)}^{-\frac{2(p+1)}{p+1}}+\left\|v_{20}\right\|_{L^{p+1}\left(\Omega_{2}^{\prime}\right)}^{-\frac{2(p+1)}{p-1}}\right) \\
= & \lim _{n \rightarrow \infty}\left(\frac{1}{2}-\frac{1}{p+1}\right)\left(\left\|v_{1 n}\right\|_{L^{p+1}\left(\Omega_{1}^{\prime}\right)}^{-\frac{2(p+1)}{p+1}}+\left\|v_{2 n}\right\|_{L^{p^{p+1}\left(\Omega_{2}^{\prime}\right)}}^{-\frac{2(p+1)}{p-1}}\right) \\
\leq & \lim _{n \rightarrow \infty} J_{\lambda_{n}}\left(p ; v_{1 n}, v_{2 n}\right) \leq 2 c_{p}\left(B_{r}\right) .
\end{aligned}
$$

Here we used the fact that

$$
\begin{aligned}
J_{\lambda}\left(p ; v_{1}, v_{2}\right) & =\sup _{s, t \geq 0} I_{\lambda}\left(p ; s v_{1}, t v_{2}\right) \geq \sup _{s, t \geq 0} \widetilde{I}_{\lambda}\left(p ; s v_{1}, t v_{2}\right) \\
& =\left(\frac{1}{2}-\frac{1}{p+1}\right)\left(\left\|v_{1}\right\|_{L^{p+1}\left(\Omega_{1}^{\prime}\right)}^{-\frac{2(p+1)}{p-1}}+\left\|v_{2}\right\|_{L^{p+1}\left(\Omega_{2}^{\prime}\right)}^{-\frac{2(p+1)}{p-1}}\right),
\end{aligned}
$$

where $\widetilde{I}_{\lambda}\left(p ; v_{1}, v_{2}\right)=\frac{1}{2}\left\|v_{1}\right\|_{\lambda, \Omega_{1}^{\prime}}^{2}+\frac{1}{2}\left\|v_{2}\right\|_{\lambda, \Omega_{2}^{\prime}}^{2}-\frac{1}{p+1}\left\|v_{1}\right\|_{L^{p+1}\left(\Omega_{1}^{\prime}\right)}^{p+1}-\frac{1}{p+1}\left\|v_{2}\right\|_{L^{p+1}\left(\Omega_{2}^{\prime}\right)}^{p+1}$. Since $c_{p}\left(\Omega_{i}\right) \leq J_{i, D}\left(\frac{v_{i 0}}{\left\|v_{i 0}\right\|_{H^{1}\left(\Omega_{i}\right)}}\right)$, we have

$$
J_{1, D}\left(\frac{v_{10}}{\left\|v_{10}\right\|_{H^{1}\left(\Omega_{1}\right)}}\right) \leq 2 c_{p}\left(B_{r}\right)-c_{p}\left(\Omega_{2}\right), \quad J_{2, D}\left(\frac{v_{20}}{\left\|v_{20}\right\|_{H^{1}\left(\Omega_{2}\right)}}\right) \leq 2 c_{p}\left(B_{r}\right)-c_{p}\left(\Omega_{1}\right) .
$$

By (6.3), we have $J_{i, D}\left(\frac{v_{i 0}}{\left\|v_{i 0}\right\|_{H^{1}\left(\Omega_{i}\right)}}\right) \leq c_{\frac{N+2}{N-2}}+\delta$. Thus by Lemma 6.4 we have $\left(\beta_{1}\left(p ; v_{10}\right), \beta_{2}\left(p ; v_{20}\right)\right) \in \Omega_{1}^{+} \times \Omega_{2}^{+}$, which is in contradiction to (6.4).

In order to prove Theorem 1.3, we need the following lemma. 
Lemma 6.6. Let $A, B, X$ be topological spaces and suppose that there exist maps $\alpha: A \rightarrow X$ and $\beta: X \rightarrow B$ such that $\beta \circ \alpha: A \rightarrow B$ is a homotopy equivalence. Then $\operatorname{cat}(A) \leq \operatorname{cat}(X)$.

Proof. Suppose that $\operatorname{cat}(X)=k$. Then there exist closed sets $X_{1}, \cdots, X_{k} \subset X$ such that $X \subset X_{1} \cup \cdots \cup X_{k}$ and each $X_{i}$ are contractible in $X$. We set $A_{i}=$ $\alpha^{-1}\left(X_{i}\right) \subset A$. It follows that

$$
\operatorname{cat}(A) \leq \sum_{i=1}^{k} \operatorname{cat}\left(A_{i}\right)
$$

We claim that, if $A_{i} \neq \emptyset, A_{i}$ is contractible in $A$; that is, $\operatorname{cat}\left(A_{i}\right)=1$. Since $X_{i}$ are contractible in $X$, there exist $H_{i} \in C\left([0,1] \times X_{i}, X\right)$ and $x_{i 0} \in X$ such that $H_{i}(0, x)=x, H_{i}(1, x)=x_{i 0}$ for all $x \in X_{i}$. Furthermore, since $\beta \circ \alpha: A \rightarrow B$ is a homotopy equivalence, there exist continuous maps $\varphi: B \rightarrow A$ and $G \in$ $C([0,1] \times A, A)$ such that $G(0, x)=x, G(1, x)=\varphi(\beta(\alpha(x)))$ for all $x \in X_{i}$. We define $F_{i} \in C\left([0,2] \times A_{i}, A\right)$ by

$$
F_{i}(t, x)= \begin{cases}G(t, x) & \text { if } t \in[0,1] \text { and } x \in A_{i}, \\ \varphi\left(\beta\left(H_{i}(t-1, \alpha(x))\right)\right) & \text { if } t \in[1,2] \text { and } x \in A_{i} .\end{cases}
$$

Then $F_{i}$ satisfies $F_{i}(0, x)=x, F_{i}(2, x)=\varphi\left(\beta\left(x_{i 0}\right)\right)$ for all $x \in A_{i}$. Therefore, $A_{i}$ is contractible in $A$; that is, $\operatorname{cat}\left(A_{i}\right)=1$. Consequently we get $\operatorname{cat}(A) \leq k=$ $\operatorname{cat}(X)$.

Set $M=2 c_{\frac{N+2}{N-2}}+1$ and let $\nu_{M}>0$ be a constant given in Proposition 9.1 and Corollary 9.3 . We set

$X_{\lambda}^{++}=\left\{\left(v_{1}, v_{2}\right) \in\left[J_{\lambda}\left(p ; v_{1}, v_{2}\right) \leq 2 c_{p}\left(B_{r}\right)\right]_{\Sigma_{1, \lambda} \times \Sigma_{2, \lambda}} ; \varphi_{-}\left(v_{1}\right) \leq \nu_{M}, \psi_{-}\left(v_{2}\right) \leq \nu_{M}\right\}$,

$X_{\lambda}^{+-}=\left\{\left(v_{1}, v_{2}\right) \in\left[J_{\lambda}\left(p ; v_{1}, v_{2}\right) \leq 2 c_{p}\left(B_{r}\right)\right]_{\Sigma_{1, \lambda} \times \Sigma_{2, \lambda}} ; \varphi_{-}\left(v_{1}\right) \leq \nu_{M}, \psi_{+}\left(v_{2}\right) \leq \nu_{M}\right\}$,

$X_{\lambda}^{-+}=\left\{\left(v_{1}, v_{2}\right) \in\left[J_{\lambda}\left(p ; v_{1}, v_{2}\right) \leq 2 c_{p}\left(B_{r}\right)\right]_{\Sigma_{1, \lambda} \times \Sigma_{2, \lambda}} ; \varphi_{+}\left(v_{1}\right) \leq \nu_{M}, \psi_{-}\left(v_{2}\right) \leq \nu_{M}\right\}$,

$X_{\lambda}^{--}=\left\{\left(v_{1}, v_{2}\right) \in\left[J_{\lambda}\left(p ; v_{1}, v_{2}\right) \leq 2 c_{p}\left(B_{r}\right)\right]_{\Sigma_{1, \lambda} \times \Sigma_{2, \lambda}} ; \varphi_{+}\left(v_{1}\right) \leq \nu_{M}, \psi_{+}\left(v_{2}\right) \leq \nu_{M}\right\}$,

where $\varphi_{ \pm}\left(v_{1}\right)=\left\|v_{1 \pm}\right\|_{L^{p+1}\left(\Omega_{1}^{\prime}\right)}^{p+1}, \psi_{ \pm}\left(v_{2}\right)=\left\|v_{2 \pm}\right\|_{L^{p+1}\left(\Omega_{2}^{\prime}\right)}^{p+1}$.

Proposition 6.7. Assume (a1)-(a2), (1.5) and $N \geq 3$. Then there exist $p_{2} \in$ $\left(1, \frac{N+2}{N-2}\right)$ and $\Lambda_{3}(p) \geq 1$ such that for $p \in\left(p_{2}, \frac{N+2}{N-2}\right)$ and $\lambda \geq \Lambda_{3}(p)$,

$$
\operatorname{cat}\left(X_{\lambda}^{\sigma_{1} \sigma_{2}}\right) \geq \operatorname{cat}\left(\Omega_{1} \times \Omega_{2}\right) \quad \text { for all } \sigma_{1}, \sigma_{2} \in\{+,-\} \text {. }
$$

Moreover $J_{\lambda}$ has at least cat $\left(\Omega_{1} \times \Omega_{2}\right)$ critical points in $X_{\lambda}^{++}\left(X_{\lambda}^{+-}, X_{\lambda}^{-+}, X_{\lambda}^{--}\right.$ respectively).

Proof. Let $\widetilde{U} \in H_{0}^{1}\left(B_{r}\right)$ be a unique solution of

$$
-\Delta u+u=u^{p} \quad \text { in } B_{r}, \quad u>0 \quad \text { in } B_{r}, \quad u=0 \quad \text { on } \partial B_{r} .
$$

We set

$$
U_{y}(x)=\frac{\widetilde{U}(x-y)}{\|\widetilde{U}\|_{\lambda, B_{r}}} \in H_{0}^{1}\left(B_{r}(y)\right) .
$$

We note that for all $(y, z) \in \Omega_{1}^{-} \times \Omega_{2}^{-}$,

$$
\begin{aligned}
& J_{\lambda}\left(p ; \sigma_{1} U_{y}, \sigma_{2} U_{z}\right)=2 c_{p}\left(B_{r}\right) \quad \text { for all } \sigma_{1}, \sigma_{2} \in\{+,-\}, \\
& \left(\beta_{1}\left(p ; \sigma_{1} U_{y}\right), \beta_{2}\left(p ; \sigma_{2} U_{z}\right)\right)=(y, z) \quad \text { for all } \sigma_{1}, \sigma_{2} \in\{+,-\} .
\end{aligned}
$$


Let $p_{2}$ and $\Lambda_{2}(p)$ be constants given in Proposition 6.5. For any $p \in\left[p_{2}, \frac{N+2}{N-2}\right)$ and $\lambda \geq \Lambda_{2}(p)$, we define two maps by

$$
\begin{aligned}
& \alpha^{\sigma_{1} \sigma_{2}}(y, z)=\left(\sigma_{1} U_{y}, \sigma_{2} U_{z}\right): \Omega_{1}^{-} \times \Omega_{2}^{-} \rightarrow X_{\lambda}^{\sigma_{1} \sigma_{2}}, \\
& \beta^{\sigma_{1} \sigma_{2}}\left(v_{1}, v_{2}\right)=\left(\beta_{1}\left(p ; v_{1}\right), \beta_{2}\left(p ; v_{2}\right)\right): X_{\lambda}^{\sigma_{1} \sigma_{2}} \rightarrow \Omega_{1}^{+} \times \Omega_{2}^{+} .
\end{aligned}
$$

By Proposition 6.5 these maps are well defined and $\beta^{\sigma_{1} \sigma_{2}} \circ \alpha^{\sigma_{1} \sigma_{2}}(y, z): \Omega_{1}^{-} \times \Omega_{2}^{-} \rightarrow$ $\Omega_{1}^{+} \times \Omega_{2}^{+}$is an identity. Therefore, from Lemma 6.6 we find

$$
\operatorname{cat}\left(X_{\lambda}^{\sigma_{1} \sigma_{2}}\right) \geq \operatorname{cat}\left(\Omega_{1}^{-} \times \Omega_{2}^{-}\right)=\operatorname{cat}\left(\Omega_{1} \times \Omega_{2}\right) .
$$

Moreover by Corollary 9.3 there exists $\Lambda_{3}(p) \geq \Lambda_{2}(p)$ such that for $\lambda \geq \Lambda_{3}(p)$ there exists a deformation $\eta(t, v)$ in $X_{\lambda}^{\sigma_{1} \sigma_{2}}$ with the following properties:

(i) $\eta(t, v) \in X_{\lambda}^{\sigma_{1} \sigma_{2}} \quad$ for all $(t, v) \in[0,1] \times X_{\lambda}^{\sigma_{1} \sigma_{2}}$,

(ii) $J_{\lambda}(\eta(t, v)) \leq J_{\lambda}(v) \quad$ for all $(t, v) \in[0,1] \times X_{\lambda}^{\sigma_{1} \sigma_{2}}$,

(iii) If $b<M \equiv 2 c_{\frac{N+2}{N-2}}+1$ is not a critical value of $J_{\lambda}$, then there exists $\epsilon>0$ such that

$$
J_{\lambda}(\eta(1, v))<b-\epsilon \quad \text { for } v \in\left[J_{\lambda}<b+\epsilon\right] .
$$

Thus $J_{\lambda}$ has at least cat $\left(\Omega_{1} \times \Omega_{2}\right)$ critical points in $X_{\lambda}^{\sigma_{1} \sigma_{2}}$.

End of the proof of Theorem [1.3. By Proposition 6.7 $J_{\lambda}$ has at least cat $\left(\Omega_{1} \times \Omega_{2}\right)$ critical points in $X_{\lambda}^{++}$. By Proposition 2.19 (iii) those critical points are positive, and by Corollary 6.3 we have inf $J_{\lambda}\left(p ; v_{1}, v_{2}\right)=c_{p}^{\lambda} \geq c_{p}\left(B_{r}\right)$. In [3], they showed that $\left(P_{\lambda}\right)$ possesses at least $\operatorname{cat}\left(\Omega_{1} \cup \Omega_{2}\right)=\operatorname{cat}\left(\Omega_{1}\right)+\operatorname{cat}\left(\Omega_{2}\right)$ positive solutions. We remark that their solutions $u(x)$ satisfy $\Psi_{\lambda}(u)<c_{p}\left(B_{r}\right)$. Thus their solutions are different from those obtained in Proposition 6.7. Thus $\left(P_{\lambda}\right)$ has at least cat $\left(\Omega_{1}\right)+$ $\operatorname{cat}\left(\Omega_{2}\right)+\operatorname{cat}\left(\Omega_{1} \times \Omega_{2}\right)$ positive solutions, and the proof of Theorem 1.3 is completed.

End of the proof of Theorem 1.5. By Proposition 6.7 $J_{\lambda}$ has at least cat $\left(\Omega_{1} \times \Omega_{2}\right)$ critical points $v_{\lambda}=\left(v_{1, \lambda}, v_{2, \lambda}\right)$ in $X_{\lambda}^{+-}$. From Propositions 2.17 and 2.19, extracting subsequence $\lambda_{n} \rightarrow \infty, v_{i, \lambda_{n}}$ converges to a critical point $v_{i \infty} \in \Sigma_{i, D}$ of $J_{i, D}(i=1,2)$ and $v_{i \infty}$ satisfies $v_{1 \infty}>0$ in $\Omega_{1}, v_{2 \infty}<0$ in $\Omega_{2}$. Thus Theorem 1.5 is proved.

\section{Proofs of Lemmas 2.9, 2.10 and Propositions 2.15, 2.16}

In this section we give proofs of various lemmas stated in Section 2 without proofs.

Proof of Lemma 2.9, Let $V(x)$ be a function satisfying (2.15). (i) is nothing but (2.16) in Lemma 2.6.

(ii) Set $v=Q_{V, \lambda}(u), w=Q_{0, \lambda}(u)$. Since $v-w \in H_{0}^{1}\left(\mathbf{R}^{N} \backslash\left(\Omega_{1}^{\prime} \cup \Omega_{2}^{\prime}\right)\right)$, we have from the variational characterization (2.18) of $v=Q_{V, \lambda}(u)$ that

$$
\langle v, v-w\rangle_{\lambda, \mathbf{R}^{N} \backslash\left(\Omega_{1}^{\prime} \cup \Omega_{2}^{\prime}\right)}-\int_{\mathbf{R}^{N} \backslash\left(\Omega_{1}^{\prime} \cup \Omega_{2}^{\prime}\right)} V(x) v(v-w) d x=0 .
$$

Similarly from the variational characterization for $w=Q_{0, \lambda}(u)$, we have

$$
\langle w, v-w\rangle_{\lambda, \mathbf{R}^{N} \backslash\left(\Omega_{1}^{\prime} \cup \Omega_{2}^{\prime}\right)}=0 .
$$


Subtracting (7.2) from (17.1),

$$
\begin{gathered}
\|v-w\|_{\lambda, \mathbf{R}^{N} \backslash\left(\Omega_{1}^{\prime} \cup \Omega_{2}^{\prime}\right)}^{2}=\int_{\mathbf{R}^{N} \backslash\left(\Omega_{1}^{\prime} \cup \Omega_{2}^{\prime}\right)} V(x) v(v-w) d x \\
\leq\left(\int_{\mathbf{R}^{N} \backslash\left(\Omega_{1}^{\prime} \cup \Omega_{2}^{\prime}\right)}|V(x)| v^{2} d x\right)^{\frac{1}{2}}\left(\int_{\mathbf{R}^{N} \backslash\left(\Omega_{1}^{\prime} \cup \Omega_{2}^{\prime}\right)}|V(x) \| v-w|^{2} d x\right)^{\frac{1}{2}} .
\end{gathered}
$$

By (2.15)

$$
\begin{aligned}
& \leq \frac{4}{9}\|v\|_{L^{2}\left(\mathbf{R}^{N} \backslash\left(\Omega_{1}^{\prime} \cup \Omega_{2}^{\prime}\right)\right)}\|v-w\|_{L^{2}\left(\mathbf{R}^{N} \backslash\left(\Omega_{1}^{\prime} \cup \Omega_{2}^{\prime}\right)\right)} \\
& \leq r_{\lambda}\|v\|_{\lambda, \mathbf{R}^{N} \backslash\left(\Omega_{1}^{\prime} \cup \Omega_{2}^{\prime}\right)}\|v-w\|_{\lambda, \mathbf{R}^{N} \backslash\left(\Omega_{1}^{\prime} \cup \Omega_{2}^{\prime}\right)} .
\end{aligned}
$$

Therefore by (i) we have

$$
\|v-w\|_{\lambda, \mathbf{R}^{N} \backslash\left(\Omega_{1}^{\prime} \cup \Omega_{2}^{\prime}\right)} \leq r_{\lambda}\|v\|_{\lambda, \mathbf{R}^{N} \backslash\left(\Omega_{1}^{\prime} \cup \Omega_{2}^{\prime}\right)} \leq r_{\lambda} C_{3}\|u\|_{\lambda, \Omega_{1}^{\prime} \cup \Omega_{2}^{\prime}} .
$$

Thus we get (ii).

(iii) For (iii) we need the following proposition.

Proposition 7.1. For any open sets $O_{1}, O_{2}$ satisfying $\Omega_{1}^{\prime} \subset \subset O_{1}, \Omega_{2}^{\prime} \subset \subset O_{2}$, there exist $c_{\lambda}\left(O_{1}\right), c_{\lambda}\left(O_{2}\right)>0$ such that

$$
\begin{aligned}
& c_{\lambda}\left(O_{1}\right), c_{\lambda}\left(O_{2}\right) \rightarrow 0 \quad \text { as } \lambda \rightarrow \infty, \\
& \left\|Q_{0, \lambda}\left(u_{1}, 0\right)\right\|_{\lambda, \mathbf{R}^{N} \backslash\left(O_{1} \cup \Omega_{2}^{\prime}\right)} \leq c_{\lambda}\left(O_{1}\right)\left\|u_{1}\right\|_{\lambda, \Omega_{1}^{\prime}} \quad \text { for all } u_{1} \in H^{1}\left(\Omega_{1}^{\prime}\right), \\
& \left\|Q_{0, \lambda}\left(0, u_{2}\right)\right\|_{\lambda, \mathbf{R}^{N} \backslash\left(\Omega_{1}^{\prime} \cup O_{2}\right)} \leq c_{\lambda}\left(O_{2}\right)\left\|u_{2}\right\|_{\lambda, \Omega_{2}^{\prime}} \text { for all } u_{2} \in H^{1}\left(\Omega_{2}^{\prime}\right) .
\end{aligned}
$$

We will give a proof of Proposition 7.1 in Section 8 .

For a proof of (iii) we take open sets $O_{1}, O_{2}$ such that $\Omega_{1}^{\prime} \subset \subset O_{1}, \Omega_{2}^{\prime} \subset \subset O_{2}$ and $O_{1} \cap O_{2}=\emptyset$. By Proposition 7.1, there exist constants $c_{\lambda}\left(O_{1}\right), c_{\lambda}\left(O_{2}\right)>0$ satisfying (7.3) $-(7.4)$.

We have

$$
\begin{aligned}
& \left\langle Q_{0, \lambda}\left(u_{1}, 0\right), Q_{0, \lambda}\left(0, u_{2}\right)\right\rangle_{\lambda, \mathbf{R}^{N} \backslash\left(\Omega_{1}^{\prime} \cup \Omega_{2}^{\prime}\right)} \\
= & \left\langle Q_{0, \lambda}\left(u_{1}, 0\right), Q_{0, \lambda}\left(0, u_{2}\right)\right\rangle_{\lambda, O_{1} \backslash \Omega_{1}^{\prime}}+\left\langle Q_{0, \lambda}\left(u_{1}, 0\right), Q_{0, \lambda}\left(0, u_{2}\right)\right\rangle_{\lambda, O_{2} \backslash \Omega_{2}^{\prime}} \\
& +\left\langle Q_{0, \lambda}\left(u_{1}, 0\right), Q_{0, \lambda}\left(0, u_{2}\right)\right\rangle_{\lambda, \mathbf{R}^{N} \backslash\left(O_{1} \cup O_{2}\right)} .
\end{aligned}
$$

By (7.3) $-(7.4)$ and (i)

$$
\begin{aligned}
& \left|\left\langle Q_{0, \lambda}\left(u_{1}, 0\right), Q_{0, \lambda}\left(0, u_{2}\right)\right\rangle_{\lambda, \mathbf{R}^{N} \backslash\left(\Omega_{1}^{\prime} \cup \Omega_{2}^{\prime}\right)}\right| \\
\leq & \left\|Q_{0, \lambda}\left(u_{1}, 0\right)\right\|_{\lambda, \mathbf{R}^{N} \backslash\left(\Omega_{1}^{\prime} \cup \Omega_{2}^{\prime}\right)}\left\|Q_{0, \lambda}\left(0, u_{2}\right)\right\|_{\lambda, \mathbf{R}^{N} \backslash\left(\Omega_{1}^{\prime} \cup O_{2}\right)} \\
& +\left\|Q_{0, \lambda}\left(u_{1}, 0\right)\right\|_{\lambda, \mathbf{R}^{N} \backslash\left(O_{1} \cup \Omega_{2}^{\prime}\right)}\left\|Q_{0, \lambda}\left(0, u_{2}\right)\right\|_{\lambda, \mathbf{R}^{N} \backslash\left(\Omega_{1}^{\prime} \cup \Omega_{2}^{\prime}\right)} \\
& +\left\|Q_{0, \lambda}\left(u_{1}, 0\right)\right\|_{\lambda, \mathbf{R}^{N} \backslash\left(O_{1} \cup \Omega_{2}^{\prime}\right)}\left\|Q_{0, \lambda}\left(0, u_{2}\right)\right\|_{\lambda, \mathbf{R}^{N} \backslash\left(\Omega_{1}^{\prime} \cup O_{2}\right)} \\
\leq & \left(C_{3} c_{\lambda}\left(O_{1}\right)+C_{3} c_{\lambda}\left(O_{2}\right)+c_{\lambda}\left(O_{1}\right) c_{\lambda}\left(O_{2}\right)\right)\left\|u_{1}\right\|_{\lambda, \Omega_{1}^{\prime}}\left\|u_{2}\right\|_{\lambda, \Omega_{2}^{\prime}} .
\end{aligned}
$$

Thus we get (iii).

(iv) Recalling Remark 2.8 (i), (iv) follows from (ii). 
Next we show

Proof of Lemma 2.10. (i) We can write

$$
\begin{aligned}
I_{\lambda}\left(u_{1}, u_{2}\right)= & \frac{1}{2}\left\|u_{1}\right\|_{\lambda, \Omega_{1}^{\prime}}^{2}+\frac{1}{2}\left\|u_{2}\right\|_{\lambda, \Omega_{2}^{\prime}}^{2}-\frac{1}{p+1}\left\|u_{1}\right\|_{L^{p+1}\left(\Omega_{1}^{\prime}\right)}^{p+1}-\frac{1}{p+1}\left\|u_{2}\right\|_{L^{p+1}\left(\Omega_{2}^{\prime}\right)}^{p+1} \\
& +\frac{1}{2}\left\|w_{\lambda}\left(u_{1}, u_{2}\right)\right\|_{\lambda, \mathbf{R}^{N} \backslash\left(\Omega_{1}^{\prime} \cup \Omega_{2}^{\prime}\right)}^{2}-\int_{\mathbf{R}^{N} \backslash\left(\Omega_{1}^{\prime} \cup \Omega_{2}^{\prime}\right)} G\left(x, w_{\lambda}\left(u_{1}, u_{2}\right)\right) d x .
\end{aligned}
$$

Recalling (2.24)-(2.25), we have

$$
\begin{aligned}
& I_{\lambda}\left(u_{1}, u_{2}\right)-I_{1, \lambda}\left(u_{1}\right)-I_{2, \lambda}\left(u_{2}\right) \\
= & \frac{1}{2}\left\|w_{\lambda}\left(u_{1}, u_{2}\right)\right\|_{\lambda, \mathbf{R}^{N} \backslash\left(\Omega_{1}^{\prime} \cup \Omega_{2}^{\prime}\right)}^{2}-\frac{1}{2}\left\|Q_{0, \lambda}\left(u_{1}, 0\right)\right\|_{\lambda, \mathbf{R}^{N} \backslash\left(\Omega_{1}^{\prime} \cup \Omega_{2}^{\prime}\right)}^{2} \\
& -\frac{1}{2}\left\|Q_{0, \lambda}\left(0, u_{2}\right)\right\|_{\lambda, \mathbf{R}^{N} \backslash\left(\Omega_{1}^{\prime} \cup \Omega_{2}^{\prime}\right)}^{2}-\int_{\mathbf{R}^{N} \backslash\left(\Omega_{1}^{\prime} \cup \Omega_{2}^{\prime}\right)} G\left(x, w_{\lambda}\left(u_{1}, u_{2}\right)\right) d x .
\end{aligned}
$$

By (2.2)-(2.3),

$$
\begin{aligned}
& \left|I_{\lambda}\left(u_{1}, u_{2}\right)-I_{1, \lambda}\left(u_{1}\right)-I_{2, \lambda}\left(u_{2}\right)\right| \\
\leq & \frac{1}{2} \mid\left\|w_{\lambda}\left(u_{1}, u_{2}\right)\right\|_{\lambda, \mathbf{R}^{N} \backslash\left(\Omega_{1}^{\prime} \cup \Omega_{2}^{\prime}\right)}^{2}-\left\|Q_{0, \lambda}\left(u_{1}, 0\right)\right\|_{\lambda, \mathbf{R}^{N} \backslash\left(\Omega_{1}^{\prime} \cup \Omega_{2}^{\prime}\right)}^{2} \\
& -\left\|Q_{0, \lambda}\left(0, u_{2}\right)\right\|_{\lambda, \mathbf{R}^{N} \backslash\left(\Omega_{1}^{\prime} \cup \Omega_{2}^{\prime}\right)}^{2} \mid+\frac{2}{3}\left\|w_{\lambda}\left(u_{1}, u_{2}\right)\right\|_{L^{2}\left(\mathbf{R}^{N} \backslash\left(\Omega_{1}^{\prime} \cup \Omega_{2}^{\prime}\right)\right)}^{2} \\
= & \frac{1}{2}|(I)|+(I I) .
\end{aligned}
$$

We also recall Remark 2.8 (i), that is,

$$
w_{\lambda}(u)=Q_{V, \lambda}(u) \text { for } V(x)=g\left(x, w_{\lambda}(u)\right) / w_{\lambda}(u),
$$

and we have

$$
\begin{aligned}
(I)= & \left\|Q_{V, \lambda}(u)-Q_{0, \lambda}(u)+Q_{0, \lambda}(u)\right\|_{\lambda, \mathbf{R}^{N} \backslash\left(\Omega_{1}^{\prime} \cup \Omega_{2}^{\prime}\right)}^{2} \\
& -\left\|Q_{0, \lambda}\left(u_{1}, 0\right)\right\|_{\lambda, \mathbf{R}^{N} \backslash\left(\Omega_{1}^{\prime} \cup \Omega_{2}^{\prime}\right)}^{2}-\left\|Q_{0, \lambda}\left(0, u_{2}\right)\right\|_{\lambda, \mathbf{R}^{N} \backslash\left(\Omega_{1}^{\prime} \cup \Omega_{2}^{\prime}\right)}^{2} \\
= & \left\|Q_{V, \lambda}(u)-Q_{0, \lambda}(u)\right\|_{\lambda, \mathbf{R}^{N} \backslash\left(\Omega_{1}^{\prime} \cup \Omega_{2}^{\prime}\right)}^{2} \\
& +2\left\langle Q_{V, \lambda}(u)-Q_{0, \lambda}(u), Q_{0, \lambda}(u)\right\rangle_{\lambda, \mathbf{R}^{N} \backslash\left(\Omega_{1}^{\prime} \cup \Omega_{2}^{\prime}\right)} \\
& +2\left\langle Q_{0, \lambda}\left(u_{1}, 0\right), Q_{0, \lambda}\left(0, u_{2}\right)\right\rangle_{\lambda, \mathbf{R}^{N} \backslash\left(\Omega_{1}^{\prime} \cup \Omega_{2}^{\prime}\right)} .
\end{aligned}
$$

Thus by Lemma 2.9.

$$
\begin{aligned}
|(I)| & \leq r_{\lambda}^{2}\|u\|_{\lambda, \Omega_{1}^{\prime} \cup \Omega_{2}^{\prime}}^{2}+2 r_{\lambda} C_{3}\|u\|_{\lambda, \Omega_{1}^{\prime} \cup \Omega_{2}^{\prime}}^{2}+2 r_{\lambda}\left\|u_{1}\right\|_{\lambda, \Omega_{1}^{\prime}}\left\|u_{2}\right\|_{\lambda, \Omega_{2}^{\prime}} \\
& \leq r_{\lambda}^{\prime}\|u\|_{\lambda, \Omega_{1}^{\prime} \cup \Omega_{2}^{\prime}}^{2} .
\end{aligned}
$$

On the other hand, using Lemma 2.1.

$$
\begin{aligned}
|(I I)| & =\frac{2}{3}\left\|Q_{V, \lambda}(u)\right\|_{L^{2}\left(\mathbf{R}^{N} \backslash\left(\Omega_{1}^{\prime} \cup \Omega_{2}^{\prime}\right)\right)}^{2} \\
& \leq \frac{2}{3} C_{2}\left(\mathbf{R}^{N} \backslash\left(\Omega_{1}^{\prime} \cup \Omega_{2}^{\prime}\right), \lambda\right)^{2}\left\|Q_{V, \lambda}(u)\right\|_{\lambda, \mathbf{R}^{N}}^{2} \backslash\left(\Omega_{1}^{\prime} \cup \Omega_{2}^{\prime}\right) \\
& \leq \frac{2}{3} C_{2}\left(\mathbf{R}^{N} \backslash\left(\Omega_{1}^{\prime} \cup \Omega_{2}^{\prime}\right), \lambda\right)^{2} C_{3}^{2}\|u\|_{\lambda, \Omega_{1}^{\prime} \cup \Omega_{2}^{\prime}}^{2} .
\end{aligned}
$$

(i) follows from (7.5) -(7.6). 
(ii) For $\varphi \in H_{0}^{1}\left(\Omega_{1}^{\prime}\right)$, we have

$$
\begin{aligned}
I_{1, \lambda}^{\prime}\left(u_{1}\right) \varphi= & \left\langle u_{1}, \varphi\right\rangle_{\lambda, \Omega_{1}^{\prime}}-\int_{\Omega_{1}^{\prime}}\left|u_{1}\right|^{p-1} u_{1} \varphi d x \\
& +\left\langle Q_{0, \lambda}\left(u_{1}, 0\right), Q_{0, \lambda}(\varphi, 0)\right\rangle_{\lambda, \mathbf{R}^{N} \backslash\left(\Omega_{1}^{\prime} \cup \Omega_{2}^{\prime}\right)}, \\
\frac{\partial I_{\lambda}}{\partial u_{1}}\left(u_{1}, u_{2}\right) \varphi= & \left\langle u_{1}, \varphi\right\rangle_{\lambda, \Omega_{1}^{\prime}}-\int_{\Omega_{1}^{\prime}}\left|u_{1}\right|^{p-1} u_{1} \varphi d x \\
& +\left\langle w_{\lambda}\left(u_{1}, u_{2}\right), \frac{\partial w_{\lambda}}{\partial u_{1}}\left(u_{1}, u_{2}\right) \varphi\right\rangle_{\lambda, \mathbf{R}^{N} \backslash\left(\Omega_{1}^{\prime} \cup \Omega_{2}^{\prime}\right)} \\
& -\int_{\mathbf{R}^{N} \backslash\left(\Omega_{1}^{\prime} \cup \Omega_{2}^{\prime}\right)} g\left(x, w_{\lambda}\left(u_{1}, u_{2}\right)\right) \frac{\partial w_{\lambda}}{\partial u_{1}}\left(u_{1}, u_{2}\right) \varphi d x .
\end{aligned}
$$

By Remark 2.8, we have $w_{\lambda}(u)=Q_{f\left(w_{\lambda}\right) / w_{\lambda}, \lambda}(u)$ and $\frac{\partial w_{\lambda}}{\partial u_{1}}\left(u_{1}, u_{2}\right) \varphi=$ $Q_{f^{\prime}\left(w_{\lambda}(u)\right), \lambda}(\varphi, 0)$ for $\varphi \in H^{1}\left(\Omega_{1}^{\prime}\right)$. Thus from (7.7)-(7.8),

$$
\begin{aligned}
& \frac{\partial I_{\lambda}}{\partial u_{1}}\left(u_{1}, u_{2}\right) \varphi-I_{1, \lambda}\left(u_{1}\right) \varphi \\
= & -\left\langle Q_{0, \lambda}\left(u_{1}, 0\right), Q_{0, \lambda}(\varphi, 0)\right\rangle_{\lambda, \mathbf{R}^{N} \backslash\left(\Omega_{1}^{\prime} \cup \Omega_{2}^{\prime}\right)} \\
& +\left\langle Q_{f\left(w_{\lambda}\right) / w_{\lambda}, \lambda}\left(u_{1}, u_{2}\right), Q_{f^{\prime}\left(w_{\lambda}\right), \lambda}(\varphi, 0)\right\rangle_{\lambda, \mathbf{R}^{N} \backslash\left(\Omega_{1}^{\prime} \cup \Omega_{2}^{\prime}\right)} \\
& +\int_{\mathbf{R}^{N} \backslash\left(\Omega_{1}^{\prime} \cup \Omega_{2}^{\prime}\right)} g\left(x, w_{\lambda}\left(u_{1}, u_{2}\right)\right) Q_{f^{\prime}\left(w_{\lambda}\right), \lambda}(\varphi, 0) d x \\
= & (I)+(I I)+(I I I) .
\end{aligned}
$$

By Lemma 2.9,

$$
|(I)+(I I)| \leq r_{\lambda}\|u\|_{\lambda, \Omega_{1}^{\prime} \cup \Omega_{2}^{\prime}}\|\varphi\|_{\lambda, \Omega_{1}^{\prime} \cup \Omega_{2}^{\prime}} .
$$

We also have from Lemma 2.1

$$
\begin{aligned}
& \left|\int_{\mathbf{R}^{N} \backslash\left(\Omega_{1}^{\prime} \cup \Omega_{2}^{\prime}\right)} g\left(x, w_{\lambda}(u)\right) Q_{f^{\prime}\left(w_{\lambda}\right), \lambda}(\varphi, 0) d x\right| \\
\leq & \frac{2}{3}\left\|w_{\lambda}(u)\right\|_{L^{2}\left(\mathbf{R}^{N} \backslash\left(\Omega_{1}^{\prime} \cup \Omega_{2}^{\prime}\right)\right)}\left\|Q_{f^{\prime}\left(w_{\lambda}\right), \lambda}(\varphi, 0)\right\|_{L^{2}\left(\mathbf{R}^{N} \backslash\left(\Omega_{1}^{\prime} \cup \Omega_{2}^{\prime}\right)\right)} \\
\leq & \frac{2}{3} C_{2}\left(\mathbf{R}^{N} \backslash\left(\Omega_{1}^{\prime} \cup \Omega_{2}^{\prime}\right), \lambda\right)^{2}\|u\|_{\lambda, \Omega_{1}^{\prime} \cup \Omega_{2}^{\prime}}\|\varphi\|_{\lambda, \Omega_{1}^{\prime} \cup \Omega_{2}^{\prime} .}
\end{aligned}
$$

Thus we have for some $r_{\lambda}^{\prime}>0$ satisfying $r_{\lambda}^{\prime} \rightarrow 0$ as $\lambda \rightarrow \infty$,

$$
\left|\frac{\partial I_{\lambda}}{\partial u_{1}}\left(u_{1}, u_{2}\right) \varphi-I_{1, \lambda}\left(u_{1}\right) \varphi\right| \leq r_{\lambda}^{\prime}\|u\|_{\lambda, \Omega_{1}^{\prime} \cup \Omega_{2}^{\prime}}\|\varphi\|_{\lambda, \Omega_{1}^{\prime}} \quad \text { for all } \varphi \in H^{1}\left(\Omega_{1}^{\prime}\right) .
$$

Therefore (ii) holds. (iii) can be shown in a similar way.

Next we give a proof of Proposition 2.15 First we observe some fundamental properties of $J_{i, \lambda}(v)$ : We can find an $M$-independent constant $R_{0}$ and constants $R_{1}(M), \delta_{M}>0$, which depend on $M>0$ but are independent of $\lambda$ such that for $i=1,2$ and $v_{i} \in \Sigma_{i, \lambda}$

(i) $J_{i, \lambda}\left(v_{i}\right) \leq M$ implies $\left\|v_{i}\right\|_{L^{p+1}\left(\Omega_{i}^{\prime}\right)} \geq \delta_{M}$.

(ii) Suppose that $J_{i, \lambda}\left(v_{i}\right) \leq M$. Then for $s \geq 0$

$$
\frac{m_{i}}{2} \leq I_{i, \lambda}\left(s v_{i}\right) \quad \text { implies } \quad R_{0} \leq s \leq R_{1}(M) \text {. }
$$


Here $m_{i}>0$ is defined in (2.29). This fact is easily seen from the definition of $J_{i, \lambda}(v)$ and (2.26).

For a proof of Proposition 2.15, we first observe that $J_{\lambda}\left(v_{1}, v_{2}\right)$ has properties similar to (i)-(ii). In what follows we fix $\lambda_{0} \geq 1$ such that

$$
r_{\lambda} \leq \frac{1}{2} \quad \text { for } \lambda \geq \lambda_{0}
$$

We can observe the following lemma easily from Corollary 2.11.

Lemma 7.2. There exist $M$-independent constants $R_{0}, \delta_{0}>0$ and constants $R_{1}(M), \delta_{M}>0$, which depend on $M>0$ but are independent of $\lambda$ such that for $\left(v_{1}, v_{2}\right) \in \Sigma_{1, \lambda} \times \Sigma_{2, \lambda}$

(i) $J_{\lambda}\left(v_{1}, v_{2}\right) \leq M$ implies $\left\|v_{1}\right\|_{L^{p+1}\left(\Omega_{1}^{\prime}\right)},\left\|v_{2}\right\|_{L^{p+1}\left(\Omega_{2}^{\prime}\right)} \in\left[\delta_{M}, \delta_{0}\right]$.

(ii) Suppose $\lambda \geq \lambda_{0}$ and $J_{\lambda}\left(v_{1}, v_{2}\right) \leq M$. Then for $(s, t) \in[0, \infty)^{2}$

$$
\frac{1}{2}\left(m_{1}+m_{2}\right) \leq I_{\lambda}\left(s v_{1}, t v_{2}\right) \quad \text { implies } \quad R_{0}^{2} \leq s^{2}+t^{2} \leq R_{1}(M)^{2} .
$$

(iii) There exists $\lambda_{1 M} \geq \lambda_{0}$ such that for $\lambda \geq \lambda_{1 M}$ and $\left(v_{1}, v_{2}\right) \in\left[J_{\lambda} \leq\right.$ $M]_{\Sigma_{1, \lambda} \times \Sigma_{2, \lambda}}$

(a) $s^{2}+t^{2} \leq R_{0}^{2}$ implies $I_{\lambda}^{\prime}\left(s v_{1}, t v_{2}\right)\left(s v_{1}, t v_{2}\right)>0$.

(b) $s^{2}+t^{2} \geq R_{1}(M)^{2}$ implies $I_{\lambda}^{\prime}\left(s v_{1}, t v_{2}\right)\left(s v_{1}, t v_{2}\right)<0$.

(c) $s=R_{0}$ and $t \in\left[0, R_{1}(M)\right]$ implies $I_{\lambda}^{\prime}\left(s v_{1}, t v_{2}\right)\left(v_{1}, 0\right)>0$.

(d) $s \in\left[0, R_{1}(M)\right]$ and $t=R_{0}$ implies $I_{\lambda}^{\prime}\left(s v_{1}, t v_{2}\right)\left(0, v_{2}\right)>0$.

(e) $J_{\lambda}\left(v_{1}, v_{2}\right)>\max \left\{\max _{0 \leq s \leq R_{0}, t \geq 0} I_{\lambda}\left(s v_{1}, t v_{2}\right), \max _{s \geq 0,0 \leq t \leq R_{0}} I_{\lambda}\left(s v_{1}, t v_{2}\right)\right\}$.

We remark that (i) of Proposition 2.15 follows from Lemma 7.2. By the above Lemma 7.2, $(s, t) \mapsto I_{\lambda}\left(s v_{1}, t v_{2}\right)$ takes its maximum in $\left\{(s, t) ; s^{2}+t^{2} \leq R_{1}(M)^{2}\right.$, $\left.s, t \geq R_{0}\right\}$. More precisely we have

Lemma 7.3. For any $M>0$ there exists $\delta_{\lambda, M}>0$ such that

(i) $\delta_{\lambda, M} \rightarrow 0$ as $\lambda \rightarrow \infty$.

(ii) For any $\left(v_{1}, v_{2}\right) \in\left[J_{\lambda} \leq M\right]_{\Sigma_{1, \lambda} \times \Sigma_{2, \lambda}}$,

$$
I_{\lambda}\left(s v_{1}, t v_{2}\right)=J_{\lambda}\left(v_{1}, v_{2}\right) \text { implies }\left|s-s_{1, \lambda}\left(v_{1}\right)\right| \leq \delta_{\lambda, M},\left|t-t_{2, \lambda}\left(v_{2}\right)\right| \leq \delta_{\lambda, M} \text {. }
$$

Here $s_{1, \lambda}\left(v_{1}\right)\left(t_{2, \lambda}\left(v_{2}\right)\right.$ respectively) is a unique critical point of $s \mapsto I_{i, \lambda}\left(s v_{i}\right)$ $\left(t \mapsto I_{2, \lambda}\left(t v_{2}\right)\right.$ respectively), which is given in (2.27).

We can show Lemma 7.3 without difficulty, and we omit the proof. We have the following uniqueness and regularity result for the maximizer.

Lemma 7.4. For any $M>0$ there exists $\lambda_{M} \geq 1$ such that

(i) For any $\lambda \geq \lambda_{M}$ and $\left(v_{1}, v_{2}\right) \in\left[J_{\lambda} \leq M\right]_{\Sigma_{1, \lambda} \times \Sigma_{2, \lambda}}$

$$
(s, t) \mapsto I_{\lambda}\left(s v_{1}, t v_{2}\right)
$$

has a unique maximum and is nondegenerate.

(ii) We denote the unique maximizer $\left(s_{\lambda}\left(v_{1}, v_{2}\right), t_{\lambda}\left(v_{1}, v_{2}\right)\right)$. Then for $\lambda \geq \lambda_{M}$

$$
\left[J_{\lambda} \leq M\right]_{\Sigma_{1, \lambda} \times \Sigma_{2, \lambda}} \rightarrow \mathbf{R}^{2} ;\left(v_{1}, v_{2}\right) \mapsto\left(s_{\lambda}\left(v_{1}, v_{2}\right), t_{\lambda}\left(v_{1}, v_{2}\right)\right)
$$

is of class $C^{1}$.

(iii) $\left[J_{\lambda}<M\right]_{\Sigma_{1, \lambda} \times \Sigma_{2, \lambda}} \rightarrow \mathbf{R} ;\left(v_{1}, v_{2}\right) \mapsto J_{\lambda}\left(v_{1}, v_{2}\right)=I_{\lambda}\left(s_{\lambda}\left(v_{1}, v_{2}\right) v_{1}, t_{\lambda}\left(v_{1}, v_{2}\right) v_{2}\right)$ is of class $C^{1}$ for $\lambda \geq \lambda_{M}$. 
Proof. For $\left(v_{1}, v_{2}\right) \in\left[J_{\lambda} \leq M\right]_{\Sigma_{1, \lambda} \times \Sigma_{2, \lambda}}$, we set $f_{\lambda}(s, t)=I_{\lambda}\left(s v_{1}, t v_{2}\right)$. By (2.23),

$$
\partial_{s} f_{\lambda}(s, t)=I_{\lambda}^{\prime}\left(s v_{1}, t v_{2}\right)\left(v_{1}, 0\right)=\Phi_{\lambda}^{\prime}\left(w_{\lambda}\left(s v_{1}, t v_{2}\right)\right) Q_{0, \lambda}\left(v_{1}, 0\right) .
$$

Thus we have

$$
\begin{aligned}
\partial_{s}^{2} f_{\lambda}(s, t) & =\Phi_{\lambda}^{\prime \prime}\left(w_{\lambda}\left(s v_{1}, t v_{2}\right)\right)\left(w_{\lambda}^{\prime}\left(s v_{1}, t v_{2}\right)\left(v_{1}, 0\right), Q_{0, \lambda}\left(v_{1}, 0\right)\right) \\
& =\Phi_{\lambda}^{\prime \prime}\left(w_{\lambda}\left(s v_{1}, t v_{2}\right)\right)\left(Q_{f^{\prime}\left(w_{\lambda}\left(s v_{1}, t v_{2}\right)\right), \lambda}\left(v_{1}, 0\right), Q_{0, \lambda}\left(v_{1}, 0\right)\right) \\
\partial_{t} \partial_{s} f_{\lambda}(s, t) & =\Phi_{\lambda}^{\prime \prime}\left(w_{\lambda}\left(s v_{1}, t v_{2}\right)\right)\left(Q_{f^{\prime}\left(w_{\lambda}\left(s v_{1}, t v_{2}\right)\right), \lambda}\left(0, v_{2}\right), Q_{0, \lambda}\left(v_{1}, 0\right)\right) .
\end{aligned}
$$

Analogously,

$$
\partial_{t}^{2} f_{\lambda}(s, t)=\Phi_{\lambda}^{\prime \prime}\left(w_{\lambda}\left(s v_{1}, t v_{2}\right)\right)\left(Q_{f^{\prime}\left(w_{\lambda}\left(s v_{1}, t v_{2}\right)\right), \lambda}\left(0, v_{2}\right), Q_{0, \lambda}\left(0, v_{2}\right)\right) .
$$

Since

$\Phi_{\lambda}^{\prime \prime}(u)\left(h_{1}, h_{2}\right)=\left\langle h_{1}, h_{2}\right\rangle_{\lambda, \mathbf{R}^{N}}-p \int_{\Omega_{1}^{\prime} \cup \Omega_{2}^{\prime}}|u|^{p-1} h_{1} h_{2} d x-\int_{\mathbf{R}^{N} \backslash\left(\Omega_{1}^{\prime} \cup \Omega_{2}^{\prime}\right)} f^{\prime}(u) h_{1} h_{2} d x$,

we have by Lemma 2.9

$$
\begin{aligned}
\partial_{s}^{2} f_{\lambda}(s, t)= & \left\langle Q_{f^{\prime}\left(w_{\lambda}\left(s v_{1}, t v_{2}\right)\right), \lambda}\left(v_{1}, 0\right), Q_{0, \lambda}\left(v_{1}, 0\right)\right\rangle_{\lambda, \mathbf{R}^{N}}-p s^{p-1}\left\|v_{1}\right\|_{L^{p+1}\left(\Omega_{1}^{\prime}\right)}^{p+1} \\
& -\int_{\mathbf{R}^{N} \backslash\left(\Omega_{1}^{\prime} \cup \Omega_{2}^{\prime}\right)} f^{\prime}\left(w_{\lambda}\left(s v_{1}, t v_{2}\right)\right) Q_{f^{\prime}\left(w_{\lambda}\left(s v_{1}, t v_{2}\right)\right), \lambda}\left(v_{1}, 0\right) Q_{0, \lambda}\left(v_{1}, 0\right) d x \\
= & \left\|v_{1}\right\|_{\lambda, \Omega_{1}^{\prime}, \#}^{2}-p s^{p-1}\left\|v_{1}\right\|_{L^{p+1}\left(\Omega_{1}^{\prime}\right)}^{p+1}+r_{\lambda}\left(s, t, v_{1}, v_{2}\right) .
\end{aligned}
$$

Here $r_{\lambda}\left(s, t, v_{1}, v_{2}\right)$ satisfies $r_{\lambda}\left(s, t, v_{1}, v_{2}\right) \rightarrow 0$ as $\lambda \rightarrow \infty$ uniformly in $\left(v_{1}, v_{2}\right) \in$ $\left[J_{\lambda} \leq M\right]_{\Sigma_{1, \lambda} \times \Sigma_{2, \lambda}}$ and $(s, t) \in\left\{(s, t) ; s^{2}+t^{2} \leq R_{1}(M)^{2}, s, t \geq R_{0}\right\}$.

Now suppose that $\left(v_{1}, v_{2}\right) \in\left[J_{\lambda} \leq M\right]_{\Sigma_{1, \lambda} \times \Sigma_{2, \lambda}}$ and $(s, t) \in\left\{(s, t) ; s^{2}+t^{2} \leq\right.$ $\left.R_{1}(M)^{2}, s, t \geq R_{0}\right\}$ is a critical point of $f_{\lambda}(s, t)$. By (7.9), for any $\epsilon>0$ there exists $\lambda_{\epsilon, M} \geq 1$ such that

$$
\left|\partial_{s}^{2} f_{\lambda}(s, t)-(1-p)\left\|v_{1}\right\|_{L^{p+1}\left(\Omega_{1}^{\prime}\right)}^{p+1}\right|<\epsilon \quad \text { for } \lambda \geq \lambda_{\epsilon, M} .
$$

In a similar way, we have

$$
\left|\partial_{t} \partial_{s} f_{\lambda}(s, t)\right|<\epsilon, \quad\left|\partial_{t}^{2} f_{\lambda}(s, t)-(1-p)\left\|v_{2}\right\|_{L^{p+1}\left(\Omega_{2}^{\prime}\right)}^{p+1}\right|<\epsilon
$$

for $\lambda \geq \lambda_{\epsilon, M}$ and for any critical point $(s, t)$ in $\left\{(s, t) ; s^{2}+t^{2} \leq R_{1}(M)^{2}, s, t \geq R_{0}\right\}$.

Recalling (i) of Lemma 7.2 and choosing $\epsilon>0$ small, we have

$$
\operatorname{det}\left[\begin{array}{cc}
\partial_{s}^{2} f_{\lambda}(s, t) & \partial_{t} \partial_{s} f_{\lambda}(s, t) \\
\partial_{t} \partial_{s} f_{\lambda}(s, t) & \partial_{t}^{2} f_{\lambda}(s, t)
\end{array}\right]>0
$$

for all critical points in $\left\{(s, t) ; R_{0}^{2} \leq s^{2}+t^{2} \leq R_{1}(M)^{2}, s, t \geq R_{0}\right\}$. Thus uniqueness of critical points follows from (iii) of Lemma 7.2 and we get (i). Since unique maximizer is nondegenerate, the conclusion (ii) follows from the implicit function theorem. (iii) follows from (ii).

Proof of (ii)-(v) of Proposition 2.15, (ii) and (iv) are nothing but (i), (iii) of Lemma 7.4, (iii) follows from Lemma 7.2 . 
Next we prove (v). For (2.32), we have

$$
\begin{aligned}
& \left|J_{\lambda}\left(v_{1}, v_{2}\right)-J_{1, \lambda}\left(v_{1}\right)-J_{2, \lambda}\left(v_{2}\right)\right| \\
= & \left|I_{\lambda}\left(s_{\lambda}\left(v_{1}, v_{2}\right) v_{1}, t_{\lambda}\left(v_{1}, v_{2}\right) v_{2}\right)-I_{1, \lambda}\left(s_{1, \lambda}\left(v_{1}\right) v_{1}\right)-I_{2, \lambda}\left(t_{2, \lambda}\left(v_{2}\right) v_{2}\right)\right| \\
\leq \quad & \left|I_{\lambda}\left(s_{\lambda}\left(v_{1}, v_{2}\right) v_{1}, t_{\lambda}\left(v_{1}, v_{2}\right) v_{2}\right)-I_{1, \lambda}\left(s_{\lambda}\left(v_{1}, v_{2}\right) v_{1}\right)-I_{2, \lambda}\left(s_{\lambda}\left(v_{1}, v_{2}\right) v_{2}\right)\right| \\
& +\left|I_{1, \lambda}\left(s_{\lambda}\left(v_{1}, v_{2}\right) v_{1}\right)-I_{1, \lambda}\left(s_{1, \lambda}\left(v_{1}\right) v_{1}\right)\right| \\
& +\left|I_{2, \lambda}\left(s_{\lambda}\left(v_{1}, v_{2}\right) v_{2}\right)-I_{2, \lambda}\left(t_{2, \lambda}\left(v_{2}\right) v_{2}\right)\right| .
\end{aligned}
$$

Thus by Lemma 2.10 and Lemma 7.3, we get (2.32). We can show (2.33) in a similar way.

Finally we give a proof of Proposition 2.16,

Proof of Proposition 2.16. Suppose $\left(v_{1 n}, v_{2 n}\right) \in\left[J_{\lambda} \leq M\right]_{\Sigma_{1, \lambda} \times \Sigma_{2, \lambda}}$ satisfies

$$
J_{\lambda}\left(v_{1 n}, v_{2 n}\right) \rightarrow c \in(-\infty, M), \quad\left\|J_{\lambda}^{\prime}\left(v_{1 n}, v_{2 n}\right)\right\|_{\left(T_{\left(v_{1 n}, v_{2 n}\right)}\left(\Sigma_{1, \lambda} \times \Sigma_{2, \lambda}\right)\right)^{*}} \rightarrow 0,
$$

where

$$
\begin{aligned}
& \left\|J_{\lambda}^{\prime}\left(v_{1}, v_{2}\right)\right\|_{\left(T_{\left(v_{1}, v_{2}\right)}\left(\Sigma_{1, \lambda} \times \Sigma_{2, \lambda}\right)\right)^{*}}=\sup \left\{\left|J_{\lambda}^{\prime}\left(v_{1}, v_{2}\right)\left(h_{1}, h_{2}\right)\right| ;\right. \\
& \left.\quad\left(h_{1}, h_{2}\right) \in H^{1}\left(\Omega_{1}^{\prime}\right) \oplus H^{1}\left(\Omega_{2}^{\prime}\right),\left\langle v_{1}, h_{1}\right\rangle_{\lambda, \Omega_{1}^{\prime}}=\left\langle v_{2}, h_{2}\right\rangle_{\lambda, \Omega_{2}^{\prime}}=0\right\} .
\end{aligned}
$$

We note

$$
J_{\lambda}^{\prime}\left(v_{1}, v_{2}\right)\left(h_{1}, h_{2}\right)=I_{\lambda}^{\prime}\left(s_{\lambda}\left(v_{1}, v_{2}\right) v_{1}, t_{\lambda}\left(v_{1}, v_{2}\right) v_{2}\right)\left(s_{\lambda}\left(v_{1}, v_{2}\right) h_{1}, t_{\lambda}\left(v_{1}, v_{2}\right) h_{2}\right)
$$

and

$$
I_{\lambda}^{\prime}\left(s_{\lambda}\left(v_{1}, v_{2}\right) v_{1}, t_{\lambda}\left(v_{1}, v_{2}\right) v_{2}\right)\left(\alpha v_{1}, \beta v_{2}\right)=0 \quad \text { for all }(\alpha, \beta) \in \mathbf{R}^{2} .
$$

Thus

$$
\begin{aligned}
& \left\|I_{\lambda}^{\prime}\left(s_{\lambda}\left(v_{1}, v_{2}\right) v_{1}, t_{\lambda}\left(v_{1}, v_{2}\right) v_{2}\right)\right\|_{\lambda, \Omega_{1}^{\prime} \cup \Omega_{2}^{\prime}}^{*} \\
\leq & \sqrt{\frac{1}{s_{\lambda}\left(v_{1}, v_{2}\right)^{2}}+\frac{1}{t_{\lambda}\left(v_{1}, v_{2}\right)^{2}}}\left\|J_{\lambda}^{\prime}\left(v_{1}, v_{2}\right)\right\|_{\left(T_{\left(v_{1}, v_{2}\right)}\left(\Sigma_{1, \lambda} \times \Sigma_{2, \lambda}\right)\right)^{*}} .
\end{aligned}
$$

Recalling (2.30)-(2.31), we have

$$
\left\|I_{\lambda}^{\prime}\left(s_{\lambda}\left(v_{1 n}, v_{2 n}\right) v_{1 n}, t_{\lambda}\left(v_{1 n}, v_{2 n}\right) v_{2 n}\right)\right\|_{\lambda, \Omega_{1}^{\prime} \cup \Omega_{2}^{\prime}}^{*} \rightarrow 0 .
$$

Thus $\left(s_{\lambda}\left(v_{1 n}, v_{2 n}\right) v_{1 n}, t_{\lambda}\left(v_{1 n}, v_{2 n}\right) v_{2 n}\right)$ is a $(P S)_{c}$-sequence for $I_{\lambda}\left(u_{1}, u_{2}\right)$. By Proposition 2.4, it has a strongly convergent subsequence in $H^{1}\left(\Omega_{1}^{\prime}\right) \oplus H^{1}\left(\Omega_{2}^{\prime}\right)$. Recalling (2.30) (2.31) again, we can see that $\left(v_{1 n}, v_{2 n}\right)$ has a strongly convergent subsequence in $\Sigma_{1, \lambda} \times \Sigma_{2, \lambda}$, and the $(P S)_{c}$-condition holds for $J_{\lambda}\left(v_{1}, v_{2}\right)$.

\section{Proofs of Propositions 3.4 and 7.1}

The aim of this section is to give proofs to Proposition 3.4 and Proposition 7.1

Suppose that a compact set $K$ and open sets $O_{1}, O_{2}, O_{3}$ satisfy the following condition:

$K \subset \subset O_{1} \subset \subset O_{2} \subset \subset O_{3}$ and $\partial K, \partial O_{i}(i=1,2,3)$ are compact and smooth.

$$
\inf _{x \in O_{3} \backslash K} a(x)>0 .
$$


For a given $\varphi(x) \in H^{1}\left(O_{1} \backslash K\right)$ and $\lambda \geq 1$ we consider the following linear elliptic problem:

$$
\begin{aligned}
& -\Delta u+\left(\lambda^{2} a(x)+1\right) u=0 \quad \text { in } O_{3} \backslash \overline{O_{1}}, \\
& u(x)=\varphi(x) \text { on } \partial O_{1}, \quad u(x)=0 \text { on } \partial O_{3}, \\
& u(x) \in H^{1}\left(O_{3} \backslash \overline{O_{1}}\right) .
\end{aligned}
$$

Obviously (8.3)- (8.5) has a unique solution — we denote it by $\left(S_{\lambda} \varphi\right)(x)$ - and $\left(S_{\lambda} \varphi\right)(x)$ can be characterized as

$$
\left\|S_{\lambda} \varphi\right\|_{\lambda, O_{3} \backslash \overline{O_{1}}} \leq\|u\|_{\lambda, O_{3} \backslash \overline{O_{1}}}
$$

for all $u \in H^{1}\left(O_{3} \backslash \overline{O_{1}}\right)$ satisfying (8.4).

The following proposition gives estimates of the solution $S_{\lambda} \varphi$ :

Proposition 8.1. Assume that $K, \mathrm{O}_{1}, \mathrm{O}_{2}, \mathrm{O}_{3}$ satisfy (8.1)-(8.2). Then there exist constants a, $C_{4}, C_{5}>0$ such that

$$
\begin{aligned}
\left\|S_{\lambda} \varphi\right\|_{\lambda, O_{3} \backslash \overline{O_{1}}} & \leq C_{4}\|\varphi\|_{\lambda, O_{1} \backslash K}, \\
\left\|S_{\lambda} \varphi\right\|_{\lambda, O_{3} \backslash \overline{O_{2}}} & \leq C_{5} e^{-a \lambda}\|\varphi\|_{\lambda, O_{1} \backslash K},
\end{aligned}
$$

for all $\lambda \geq 1$ and $\varphi \in H^{1}\left(O_{1} \backslash K\right)$.

We postpone the proof of Proposition 8.1 until the end of this section. As an immediate corollary to Proposition 8.1, we have

Corollary 8.2. Assume (8.1) (8.2). Then there exists a family of linear bounded operators $P_{\lambda}: H^{1}\left(O_{3} \backslash K\right) \rightarrow H^{1}\left(O_{3} \backslash K\right)$ such that

(i) For all $\lambda \geq 1$,

$$
\begin{aligned}
& \left(P_{\lambda} u\right)(x)=u(x) \quad \text { for all } x \in O_{1} \backslash K, \\
& \left(P_{\lambda} u\right)(x)=0 \quad \text { for all } x \in O_{3} \backslash \overline{O_{2}}
\end{aligned}
$$

(ii) There exists a family of positive constants $\epsilon_{\lambda}>0$ independent of $u$ such that

$$
\begin{aligned}
& \epsilon_{\lambda} \rightarrow 0 \quad \text { as } \lambda \rightarrow \infty \\
& \left\|P_{\lambda} u\right\|_{\lambda, O_{3} \backslash K} \leq\left(1+\epsilon_{\lambda}\right)\|u\|_{\lambda, O_{3} \backslash K} \quad \text { for all } \lambda \geq 1 \text { and } u \in H^{1}\left(O_{3} \backslash K\right) .
\end{aligned}
$$

Proof. Choose an open set $D$ such that $O_{1} \subset \subset D \subset \subset O_{2} \subset \subset O_{3}$. Applying Proposition 8.1 to $K, O_{1}, D, O_{3}$, we find a solution operator $S_{\lambda}: H^{1}\left(O_{1} \backslash K\right) \rightarrow$ $H^{1}\left(O_{3} \backslash K\right)$ such that

$$
\begin{aligned}
\left\|S_{\lambda} u\right\|_{\lambda, O_{3} \backslash K} & \leq\|u\|_{\lambda, O_{3} \backslash K}, \\
\left\|S_{\lambda} u\right\|_{\lambda, O_{3} \backslash \bar{D}} & \leq C_{5} e^{-a \lambda}\|u\|_{\lambda, O_{3} \backslash K},
\end{aligned}
$$

for some constant $a>0$. Here we use the same notation $S_{\lambda}$ for an operator $u \mapsto S_{\lambda}\left(\left.u\right|_{O_{1} \backslash K}\right) ; H^{1}\left(O_{3} \backslash K\right) \rightarrow H^{1}\left(O_{3} \backslash K\right)$.

We choose a function $\zeta(x) \in C^{\infty}\left(\mathbf{R}^{N},[0,1]\right)$ such that

$$
\zeta(x)= \begin{cases}1 & \text { for all } x \in D, \\ 0 & \text { for all } x \in \mathbf{R}^{N} \backslash \overline{O_{2}} .\end{cases}
$$


Setting $\left(P_{\lambda} u\right)(x)=\zeta(x)\left(S_{\lambda} u\right)(x)$, we have (8.9)-8.12). In fact, (8.9)-8.10) are clear. For (8.11) - (8.12), first we observe by (8.14)

$$
\begin{aligned}
\left\|\zeta S_{\lambda} u\right\|_{\lambda, O_{3} \backslash \bar{D}}^{2}= & \int_{O_{3} \backslash \bar{D}}\left|\nabla\left(\zeta S_{\lambda} u\right)\right|^{2}+\left(\lambda^{2} a(x)+1\right) \zeta^{2}\left|S_{\lambda} u\right|^{2} d x \\
\leq & 2 \int_{O_{3} \backslash \bar{D}}|\nabla \zeta|^{2}\left|S_{\lambda} u\right|^{2}+|\zeta|^{2}\left|\nabla\left(S_{\lambda} u\right)\right|^{2} d x \\
& +\int_{O_{3} \backslash \bar{D}}\left(\lambda^{2} a(x)+1\right)\left|S_{\lambda} u\right|^{2} d x \\
\leq & 2\left(1+\|\nabla \zeta\|_{L^{\infty}\left(\mathbf{R}^{N}\right)}^{2}\right)\left\|S_{\lambda} u\right\|_{\lambda, O_{3} \backslash \bar{D}}^{2} \\
\leq & 2\left(1+\|\nabla \zeta\|_{L^{\infty}\left(\mathbf{R}^{N}\right)}^{2}\right) C_{5}^{2} e^{-2 a \lambda}\|u\|_{\lambda, O_{3} \backslash K}^{2} .
\end{aligned}
$$

Thus by (8.13) and (8.15)

$$
\begin{aligned}
\left\|P_{\lambda} u\right\|_{\lambda, O_{3} \backslash K}^{2} & =\left\|S_{\lambda} u\right\|_{\lambda, D \backslash K}^{2}+\left\|\zeta S_{\lambda} u\right\|_{\lambda, O_{3} \backslash \bar{D}}^{2} \\
& \leq\|u\|_{\lambda, O_{3} \backslash K}^{2}+2\left(1+\|\nabla \zeta\|_{L^{\infty}\left(\mathbf{R}^{N}\right)}^{2}\right) C_{5}^{2} e^{-2 a \lambda}\|u\|_{\lambda, O_{3} \backslash K}^{2} .
\end{aligned}
$$

Thus we get (8.11)-(8.12).

Now we are in a position to prove Proposition 3.4 .

Proof of Proposition 3.4. We choose an open set $D$ such that

$$
\Omega_{2} \subset \subset D \subset \subset \tilde{\tilde{\Omega}} \subset \subset \tilde{\Omega} \subset \subset \Omega_{2}^{\prime} .
$$

We apply Corollary 8.2 to $\left(K, O_{1}, O_{2}, O_{3}\right)=\left(\bar{D}, \tilde{\tilde{\Omega}}, \tilde{\Omega}, \Omega_{2}^{\prime}\right)$, and we find a family of operators $P_{\lambda}: H^{1}\left(\Omega_{2}^{\prime} \backslash \bar{D}\right) \rightarrow H^{1}\left(\Omega_{2}^{\prime} \backslash \bar{D}\right)$ satisfying (8.9)-(8.12). Defining $F_{\lambda}:\left\{v \in \Sigma_{2, \lambda} ;\|v\|_{L^{p+1}\left(\Omega_{2}^{\prime}\right)} \geq \tilde{m}\right\} \rightarrow H_{0}^{1}(\tilde{\Omega})$ by

$$
\left(F_{\lambda} u\right)(x)= \begin{cases}u(x) & \text { for } x \in \bar{D}, \\ \left(P_{\lambda} u\right)(x) & \text { for } x \in \tilde{\Omega} \backslash \bar{D},\end{cases}
$$

$1^{\circ}, 2^{\circ}$ of Proposition 3.4 follow from (8.9) 8.12 .

For $3^{\circ}$, first we remark for $v \in \Sigma_{2, \lambda}$ that $\|v\|_{\lambda, \Omega_{2}^{\prime} \backslash \tilde{\Omega}} \leq\|v\|_{\lambda, \Omega_{2}^{\prime}}=1$. Thus

$$
\begin{aligned}
\left\|F_{\lambda} v\right\|_{L^{p+1}\left(\Omega_{2}^{\prime}\right)}^{p+1} & =\left\|F_{\lambda} v\right\|_{L^{p+1}(\tilde{\Omega})}^{p+1} \geq\|v\|_{L^{p+1}(\tilde{\tilde{\Omega}})}^{p+1}=\|v\|_{L^{p+1}\left(\Omega_{2}^{\prime}\right)}^{p+1}-\|v\|_{L^{p+1}\left(\Omega_{2}^{\prime} \backslash \tilde{\tilde{\Omega}}\right)}^{p+1} \\
& \geq\|v\|_{L^{p+1}\left(\Omega_{2}^{\prime}\right)}^{p+1}-C_{p+1}\left(\Omega_{2}^{\prime} \backslash \tilde{\tilde{\Omega}}, \lambda\right)\|v\|_{\lambda, \Omega_{2}^{\prime} \backslash \tilde{\tilde{\Omega}}}^{2} \\
& \geq\|v\|_{L^{p+1}\left(\Omega_{2}^{\prime}\right)}^{p+1}-C_{p+1}\left(\Omega_{2}^{\prime} \backslash \tilde{\tilde{\Omega}}, \lambda\right) \\
& \geq\left(1-\frac{C_{p+1}\left(\Omega_{2}^{\prime} \backslash \tilde{\tilde{\Omega}}, \lambda\right)}{\tilde{m}}\right)\|v\|_{L^{p+1}\left(\Omega_{2}^{\prime}\right)}^{p+1} .
\end{aligned}
$$

Therefore, $3^{\circ}$ also holds for large $\lambda$.

Proof of Proposition 7.1. We choose an open set $D$ such that $\Omega_{1} \subset \subset D \subset \subset \Omega_{1}^{\prime}$ $\subset \subset O_{1}$. Applying Proposition 8.1 with $\left(K, O_{1}, O_{2}, O_{3}\right)=\left(\bar{D}, \Omega_{1}^{\prime}, O_{1}, \mathbf{R}^{N} \backslash \Omega_{2}^{\prime}\right)$, we have for some constant $a>0$

$$
\left\|Q_{0, \lambda}\left(u_{1}, 0\right)\right\|_{\lambda, \mathbf{R}^{N} \backslash\left(O_{1} \cup \Omega_{2}^{\prime}\right)} \leq C_{5} e^{-a \lambda}\left\|u_{1}\right\|_{\lambda, \Omega_{1}^{\prime} \backslash \bar{D}} \leq C_{5} e^{-a \lambda}\left\|u_{1}\right\|_{\lambda, \Omega_{1}^{\prime}}
$$

for all $\lambda \geq 1$ and $u_{1} \in H^{1}\left(\Omega_{1}^{\prime}\right)$. Thus we have (7.3). We can show (7.4) analogously. 
Proof of Proposition 8.1. We choose open sets $D_{1}, D_{2}, E_{1}, E_{2}, E_{3}$ such that

$$
K \subset \subset O_{1} \subset \subset D_{1} \subset \subset D_{2} \subset \subset O_{2} \subset \subset E_{3} \subset \subset E_{2} \subset \subset E_{1} \subset \subset O_{3} .
$$

In what follows, for $\varphi \in H^{1}\left(O_{1} \backslash K\right)$ we denote $u(x)=\left(S_{\lambda} \varphi\right)(x)$, that is, $u(x)$ solves (8.3)-(8.5). First we remark that using the characterization (8.6) we can prove (8.7) as in Lemma 2.5 .

The proof of (8.8) consists of 4 steps. As the first step we show

Step 1: There exists a constant $C_{6}>0$ independent of $\varphi \in H^{1}\left(O_{1} \backslash K\right)$ such that

$$
\max _{x \in \bar{E}_{1 \backslash D_{1}}}|u(x)| \leq C_{6}\|\varphi\|_{\lambda, O_{1} \backslash K} .
$$

We choose $r>0$ such that $\overline{B\left(x_{0}, r\right)} \subset O_{3} \backslash \overline{O_{1}}$ for all $x_{0} \in \overline{E_{1}} \backslash D_{1}$, where $B\left(x_{0}, r\right)=\left\{y \in \mathbf{R}^{N} ;\left|y-x_{0}\right|<r\right\}$. By the subsolution estimate (22], Theorem C.1.2), we have for some constant $C_{7}>0$ independent of $\lambda, x_{0}, \varphi$

$$
\begin{aligned}
\left|u\left(x_{0}\right)\right| & \leq C_{7} \int_{B\left(x_{0}, r\right)}|u(x)| d x \leq C_{7} \sqrt{\operatorname{meas}\left(B\left(x_{0}, r\right)\right)}\|u\|_{L^{2}\left(B\left(x_{0}, r\right)\right)} \\
& \leq C_{7} \sqrt{\operatorname{meas}\left(B\left(x_{0}, r\right)\right)}\|u\|_{\lambda, O_{3} \backslash \overline{O_{1}}} \leq C_{4} C_{7} \sqrt{\operatorname{meas}\left(B\left(x_{0}, r\right)\right)}\|\varphi\|_{\lambda, O_{1} \backslash K} .
\end{aligned}
$$

Thus (8.16) holds.

Step 2: There exists $\mu_{1}>0$ independent of $\lambda$ and $\varphi$ such that

$$
\max _{x \in \bar{E}_{2} \backslash D_{2}}|u(x)| \leq e^{-\mu_{1} \lambda}\|\varphi\|_{\lambda, O_{1} \backslash K} .
$$

We choose $b \in\left(0, \inf _{x \in O_{3} \backslash K} a(x)\right)$. From (8.3) it holds that

$$
\left(-\Delta+2 \lambda^{2} b\right) u^{2}=-2\left(\lambda^{2} a(x)+1-\lambda^{2} b\right) u^{2}-2|\nabla u|^{2} .
$$

In particular, we have

$$
\left(-\Delta+2 \lambda^{2} b\right) u^{2} \leq 0 .
$$

We choose $r>0$ such that $\overline{B\left(x_{0}, r\right)} \subset \overline{E_{1}} \backslash D_{1}$ for all $x_{0} \in \bar{E}_{2} \backslash D_{2}$. Now we consider a solution $w(x)$ of

$$
-\Delta w+2 \lambda^{2} b w=0 \quad \text { in } B\left(x_{0}, r\right), \quad w=\left(C_{6}\|\varphi\|_{\lambda, O_{1} \backslash K}\right)^{2} \quad \text { on } \partial B\left(x_{0}, r\right) .
$$

Then we have for some $b^{\prime}>0$

$$
\left|w\left(x_{0}\right)\right| \leq C_{6}^{2} e^{-b^{\prime} \lambda}\|\varphi\|_{\lambda, O_{1} \backslash K}^{2} \quad \text { for all } \lambda \geq 1 .
$$

Comparing (8.18) and (8.19), the conclusion (8.17) follows from Step 1.

Step 3: There exists $\mu_{2}>0$ independent of $\lambda$ and $\varphi$ such that

$$
\|u\|_{\lambda, E_{3} \backslash \overline{O_{2}}} \leq e^{-\mu_{2} \lambda}\|\varphi\|_{\lambda, O_{1} \backslash K} .
$$

Choose $\psi(x) \in C_{0}^{\infty}\left(\mathbf{R}^{N},[0,1]\right)$ such that

$$
\psi(x)= \begin{cases}1 & \text { for } x \in E_{3} \backslash \overline{O_{2}}, \\ 0 & \text { for } x \in \mathbf{R}^{N} \backslash\left(\overline{E_{2}} \backslash D_{2}\right) .\end{cases}
$$

Multiplying $\psi u$ to (8.3) and integrating, we have

$$
\int_{O_{3} \backslash K} \nabla u \nabla(\psi u)+\left(\lambda^{2} a(x)+1\right) \psi u^{2} d x=0 .
$$


Thus, by (8.17) and (8.7),

$$
\begin{aligned}
\|u\|_{\lambda, E_{3} \backslash \overline{O_{2}}}^{2} & \leq \int_{E_{2} \backslash \overline{D_{2}}}|\nabla \psi\|u\| \nabla u| d x \\
& \leq C_{8}\|\nabla \psi\|_{L^{\infty}\left(\mathbf{R}^{N}\right)}\left(\max _{x \in \overline{E_{2} \backslash D_{2}}}|u(x)|\right)\|u\|_{\lambda, O_{3} \backslash \overline{O_{1}}} \\
& \leq C_{8} C_{4} e^{-\mu_{1} \lambda}\|\nabla \psi\|_{L^{\infty}\left(\mathbf{R}^{N}\right)}\|\varphi\|_{\lambda, O_{1} \backslash K}^{2} .
\end{aligned}
$$

Thus we get (8.20).

Step 4: Conclusion.

From the characterization (8.6), it follows that

$$
\|u\|_{\lambda, O_{3} \backslash \overline{O_{2}}} \leq\|w\|_{\lambda, O_{3} \backslash \overline{O_{2}}}
$$

for all $w \in H^{1}\left(O_{3} \backslash \overline{O_{2}}\right)$ satisfying $w=u$ on $\partial O_{2}$ and $w=0$ on $\partial O_{3}$. In particular, setting $w=\zeta u$, where $\zeta(x) \in C^{\infty}\left(\mathbf{R}^{N},[0,1]\right)$ is a function such that

$$
\zeta(x)= \begin{cases}0 & \text { for } x \in O_{3} \backslash \overline{E_{3}}, \\ 1 & \text { in a neighborhood of } \partial O_{2},\end{cases}
$$

we can deduce as in the proof of Corollary 8.2 that

$$
\|u\|_{\lambda, O_{3} \backslash \overline{O_{2}}} \leq\|\zeta u\|_{\lambda, E_{3} \backslash \overline{O_{2}}} \leq C_{9}\|u\|_{\lambda, E_{3} \backslash \overline{O_{2}}} \leq C_{9} e^{-\mu_{2} \lambda}\|\varphi\|_{\lambda, O_{1} \backslash K} .
$$

Thus the proof of Proposition 8.1 is completed.

\section{Construction of a Deformation flow}

The aim of this section is to construct a deformation flow which enables us to find critical points whose limits are positive or negative in $\Omega_{i}$.

We define

$$
\begin{aligned}
& \varphi_{ \pm}\left(v_{1}\right)=\left\|v_{1 \pm}\right\|_{L^{p+1}\left(\Omega_{1}^{\prime}\right)}^{p+1}: \Sigma_{1, \lambda} \rightarrow \mathbf{R}, \\
& \psi_{ \pm}\left(v_{2}\right)=\left\|v_{2 \pm}\right\|_{L^{p+1}\left(\Omega_{2}^{\prime}\right)}^{p+1}: \Sigma_{2, \lambda} \rightarrow \mathbf{R},
\end{aligned}
$$

and for $a<b$, we use the following notation:

$$
\begin{aligned}
& {\left[a \leq \varphi_{ \pm} \leq b\right]_{\Sigma_{1, \lambda} \times \Sigma_{2, \lambda}}=\left\{\left(v_{1}, v_{2}\right) \in \Sigma_{1, \lambda} \times \Sigma_{2, \lambda} ; a \leq \varphi_{ \pm}\left(v_{1}\right) \leq b\right\}} \\
& {\left[a \leq \psi_{ \pm} \leq b\right]_{\Sigma_{1, \lambda} \times \Sigma_{2, \lambda}}=\left\{\left(v_{1}, v_{2}\right) \in \Sigma_{1, \lambda} \times \Sigma_{2, \lambda} ; a \leq \psi_{ \pm}\left(v_{2}\right) \leq b\right\} .}
\end{aligned}
$$

First we have

Proposition 9.1. For $M>0$ and $\sigma_{1}, \sigma_{2} \in\{+,-\}$, there exist $\lambda_{M}>1, \nu_{M} \in$ $\left(0, \delta_{0}(M)\right]$ such that for any $\lambda \geq \lambda_{M}$, if $b<M$ is not a critical value of $J_{\lambda}$, then there exist $\epsilon, \mu>0$ and a vector field $X(v)$ on $\left[J_{\lambda} \leq M\right]_{\Sigma_{1, \lambda} \times \Sigma_{2, \lambda}}$ with the following properties:

(i) $X(v)=\left(X_{1}(v), X_{2}(v)\right) \in T_{v}\left(\Sigma_{1, \lambda} \times \Sigma_{2, \lambda}\right)$ for all $v \in\left[J_{\lambda} \leq M\right]$ and $v \mapsto$ $X(v)$ is locally Lipschitz continuous.

(ii) $J_{\lambda}^{\prime}(v) X(v) \leq-\mu$ for all $v \in\left[b-\epsilon \leq J_{\lambda} \leq b+\epsilon\right]_{\Sigma_{1, \lambda} \times \Sigma_{2, \lambda}}$.

(iii) $X(v)=0$ for all $v \in\left[J_{\lambda} \leq b-2 \epsilon\right]_{\Sigma_{1, \lambda} \times \Sigma_{2, \lambda}}$.

(iv) $J_{\lambda}^{\prime}(v) X(v) \leq 0$ for all $v \in\left[J_{\lambda} \leq M\right]_{\Sigma_{1, \lambda} \times \Sigma_{2, \lambda}}$. 
(v)

$\varphi_{\sigma_{1}}^{\prime}\left(v_{1}\right) X_{1}(v) \leq 0$ for all $v \in\left[J_{\lambda} \leq M\right]_{\Sigma_{1, \lambda} \times \Sigma_{2, \lambda}} \cap\left[\nu_{M} \leq \varphi_{\sigma_{1}} \leq 2 \nu_{M}\right]_{\Sigma_{1, \lambda} \times \Sigma_{2, \lambda}}$,

$\psi_{\sigma_{2}}^{\prime}\left(v_{2}\right) X_{2}(v) \leq 0$ for all $v \in\left[J_{\lambda} \leq M\right]_{\Sigma_{1, \lambda} \times \Sigma_{2, \lambda}} \cap\left[\nu_{M} \leq \psi_{\sigma_{2}} \leq 2 \nu_{M}\right]_{\Sigma_{1, \lambda} \times \Sigma_{2, \lambda}}$.

Here we remark that $\delta_{0}(M)>0$ is given in Proposition 2.19,

To prove Proposition 9.1 we define

$$
\begin{array}{ll}
Y_{1}^{ \pm}\left(v_{1}\right)=-v_{1 \pm}+\left\|v_{1 \pm}\right\|_{\lambda, \Omega_{1}^{\prime}}^{2} v_{1} \in T_{v_{1}}\left(\Sigma_{1, \lambda}\right) & \text { for } v_{1} \in \Sigma_{1, \lambda} \\
Y_{2}^{ \pm}\left(v_{2}\right)=-v_{2 \pm}+\left\|v_{2 \pm}\right\|_{\lambda, \Omega_{2}^{\prime}}^{2} v_{2} \in T_{v_{2}}\left(\Sigma_{2, \lambda}\right) & \text { for } v_{2} \in \Sigma_{2, \lambda} .
\end{array}
$$

We have the following.

Lemma 9.2. For $M>0$, there exist $\lambda_{M}>1, \nu_{M} \in\left(0, \delta_{0}(M)\right]$ and $\rho_{M}>0$ such that for any $\lambda \geq \lambda_{M}, \sigma_{1}, \sigma_{2} \in\{+,-\}$,

(i) for $v \in\left[J_{\lambda} \leq M\right]_{\Sigma_{1, \lambda} \times \Sigma_{2, \lambda}} \cap\left[\frac{1}{2} \nu_{M} \leq \varphi_{\sigma_{1}} \leq 2 \nu_{M}\right]_{\Sigma_{1, \lambda} \times \Sigma_{2, \lambda}}$,

$$
J_{\lambda}^{\prime}(v)\left(Y_{1}^{\sigma_{1}}(v), 0\right) \leq-\rho_{M}, \quad \varphi_{\sigma_{1}}^{\prime}\left(v_{1}\right) Y_{1}^{\sigma_{1}}(v) \leq-\rho_{M} ;
$$

(ii) for $v \in\left[J_{\lambda} \leq M\right]_{\Sigma_{1, \lambda} \times \Sigma_{2, \lambda}} \cap\left[\frac{1}{2} \nu_{M} \leq \psi_{\sigma_{2}} \leq 2 \nu_{M}\right]_{\Sigma_{1, \lambda} \times \Sigma_{2, \lambda}}$,

$$
J_{\lambda}^{\prime}(v)\left(0, Y_{2}^{\sigma_{2}}(v)\right) \leq-\rho_{M}, \quad \psi_{\sigma_{2}}^{\prime}\left(v_{2}\right) Y_{2}^{\sigma_{2}}(v) \leq-\rho_{M} .
$$

Proof. We only prove (i) with $\sigma_{1}=+$. We can deal with other cases in a similar way. For $v \in\left[J_{\lambda} \leq M\right]_{\Sigma_{1, \lambda} \times \Sigma_{2, \lambda}}$,

$$
\begin{aligned}
\varphi_{+}^{\prime}\left(v_{1}\right) Y_{1}^{+}(v) & =(p+1) \int_{\Omega_{1}^{\prime}}\left(v_{1+}\right)^{p} Y_{1}^{+}(v) d x \\
& =-(p+1)\left(1-\left\|v_{1+}\right\|_{\lambda, \Omega_{1}^{\prime}}^{2}\right)\left\|v_{1+}\right\|_{L^{p+1}\left(\Omega_{1}^{\prime}\right)}^{p+1} \\
& \leq-(p+1)\left(1-C^{-2}\left\|v_{1+}\right\|_{L^{p+1}\left(\Omega_{1}^{\prime}\right)}^{2}\right)\left\|v_{1+}\right\|_{L^{p+1}\left(\Omega_{1}^{\prime}\right)}^{p+1} .
\end{aligned}
$$

Here we use the inequality $\left\|v_{1+}\right\|_{L^{p+1}\left(\Omega_{1}^{\prime}\right)} \leq C\left\|v_{1+}\right\|_{\lambda, \Omega_{1}^{\prime}}$.

Writing $s=s_{\lambda}\left(v_{1}, v_{2}\right), t=t_{\lambda}\left(v_{1}, v_{2}\right)$, by Lemma 2.10 and Proposition 2.15 we have

$$
\begin{aligned}
& J_{\lambda}^{\prime}\left(v_{1}, v_{2}\right)\left(Y_{1}^{+}(v), 0\right) \\
= & I_{\lambda}^{\prime}\left(s v_{1}, t v_{2}\right)\left(s Y_{1}^{+}(v), 0\right)=-I_{\lambda}^{\prime}\left(s v_{1}, t v_{2}\right)\left(s v_{1+}, 0\right) \\
\leq & -I_{1, \lambda}^{\prime}\left(s v_{1}\right)\left(s v_{1+}\right)+r_{\lambda}\left(\left\|s v_{1}\right\|_{\lambda, \Omega_{1}^{\prime}}+\left\|t v_{2}\right\|_{\lambda, \Omega_{2}^{\prime}}\right)\left\|s v_{1+}\right\|_{\lambda, \Omega_{1}^{\prime}} \\
= & -s^{2}\left\|v_{1+}\right\|_{\lambda, \Omega_{1}^{\prime}, \#}^{2}+s^{p+1}\left\|v_{1+}\right\|_{L^{p+1}\left(\Omega_{1}^{\prime}\right)}^{p+1}+r_{\lambda}(s+t) s \\
\leq & -s^{2} C^{-2}\left\|v_{1+}\right\|_{L^{p+1}\left(\Omega_{1}^{\prime}\right)}^{2}+s^{p+1}\left\|v_{1+}\right\|_{L^{p+1}\left(\Omega_{1}^{\prime}\right)}^{p-1}+r_{\lambda} s(s+t) \\
\leq & -R_{0}^{2} C^{-2}\left\|v_{1+}\right\|_{L^{p+1}\left(\Omega_{1}^{\prime}\right)}^{2}+R_{1}(M)^{p+1}\left\|v_{1+}\right\|_{L^{p+1}\left(\Omega_{1}^{\prime}\right)}^{p-1}+2 r_{\lambda} R_{1}(M)^{2} .
\end{aligned}
$$

From (9.2) and (9.3), we can find $\nu_{M}>0$ and $\rho_{M}>0$ such that if $\varphi_{+}\left(v_{1}\right)=$ $\left\|v_{1+}\right\|_{L^{p+1}\left(\Omega_{1}^{\prime}\right)}^{p+1} \in\left[\frac{\nu_{M}}{2}, 2 \nu_{M}\right]$, (9.1) holds.

Proof of Proposition 9.1. Assume that $b<M$ is not a critical value. In a standard way, we can find a pseudo-gradient vector field $\tilde{X}(v)$ satisfying (i)-(iv) of Proposition 9.1 .

We choose smooth functions $\zeta: \mathbf{R} \rightarrow[0,1]$ such that $\zeta(s)=0$ for $s \leq \frac{1}{2} \nu_{M}, \zeta(s)=$ 1 for $s \geq \nu_{M}$ and $\xi: \mathbf{R} \rightarrow[0,1]$ such that $\xi(s)=0$ for $s \leq b-2 \epsilon, \xi(s)=1$ for 
$s \geq b-\epsilon$ and set

$$
\begin{aligned}
X(v)= & \left(1-\zeta\left(\varphi_{\sigma_{1}}\left(v_{1}\right)\right)\right)\left(1-\zeta\left(\psi_{\sigma_{2}}\left(v_{2}\right)\right)\right) \tilde{X}(v) \\
& +\xi\left(J_{\lambda}\left(v_{1}, v_{2}\right)\right)\left(\zeta\left(\varphi_{\sigma_{1}}\left(v_{1}\right)\right)\left(Y_{1}^{\sigma_{1}}\left(v_{1}\right), 0\right)+\zeta\left(\psi_{\sigma_{2}}\left(v_{2}\right)\right)\left(0, Y_{2}^{\sigma_{1}}\left(v_{2}\right)\right)\right) .
\end{aligned}
$$

Then we can easily observe that $X(v)$ has the desired properties (i)-(v) of Proposition 9.1

As an immediate corollary to Proposition 9.1, we have

Corollary 9.3. For $M>0$ and $\sigma_{1}, \sigma_{2} \in\{+,-\}$, there exist $\lambda_{M}>1, \nu_{M} \in$ $\left(0, \delta_{0}(M)\right]$ such that for any $\lambda \geq \lambda_{M}$, if $b<M$ is not a critical value of $J_{\lambda}$, then there exist $\epsilon>0$ and a continuous mapping $\eta(t, v):[0,1] \times\left[J_{\lambda} \leq M\right]_{\Sigma_{1, \lambda} \times \Sigma_{2, \lambda}} \rightarrow$ $\left[J_{\lambda} \leq M\right]_{\Sigma_{1, \lambda} \times \Sigma_{2, \lambda}}$ such that

(i) $J_{\lambda}(\eta(t, v)) \leq J_{\lambda}(v)$ for all $(t, v) \in[0,1] \times\left[J_{\lambda} \leq M\right]_{\Sigma_{1, \lambda} \times \Sigma_{2, \lambda}}$,

(ii) $J_{\lambda}(\eta(1, v)) \leq b-\epsilon$ for all $v \in\left[J_{\lambda} \leq b+\epsilon\right]_{\Sigma_{1, \lambda} \times \Sigma_{2, \lambda}}$,

(iii) $\eta(t, v) \in\left[J_{\lambda} \leq M\right]_{\Sigma_{1, \lambda} \times \Sigma_{2, \lambda}} \cap\left[\varphi_{\sigma_{1}} \leq \nu_{M}\right]_{\Sigma_{1, \lambda} \times \Sigma_{2, \lambda}}$ for all $(t, v) \in[0,1] \times$ $\left(\left[J_{\lambda} \leq M\right]_{\Sigma_{1, \lambda} \times \Sigma_{2, \lambda}} \cap\left[\varphi_{\sigma_{1}} \leq \nu_{M}\right]_{\Sigma_{1, \lambda} \times \Sigma_{2, \lambda}}\right)$,

(iv) $\eta(t, v) \in\left[J_{\lambda} \leq M\right]_{\Sigma_{1, \lambda} \times \Sigma_{2, \lambda}} \cap\left[\psi_{\sigma_{2}} \leq \nu_{M}\right]_{\Sigma_{1, \lambda} \times \Sigma_{2, \lambda}}$ for all $(t, v) \in[0,1] \times$ $\left(\left[J_{\lambda} \leq M\right]_{\Sigma_{1, \lambda} \times \Sigma_{2, \lambda}} \cap\left[\psi_{\sigma_{2}} \leq \nu_{M}\right]_{\Sigma_{1, \lambda} \times \Sigma_{2, \lambda}}\right)$.

\section{ACKNOWLEDGEMENTS}

The authors would like to thank Professor E. N. Dancer and Professor E. Séré for helpful discussions on isolatedness and Sard's property of critical values.

\section{REFERENCES}

1. A. Ambrosetti, A perturbation theorem for superlinear boundary value problems, MRC Univ of Wisconsin-Madison, Tech. Sum. Report 1446 (1974).

2. T. Bartsch and Z.-Q. Wang, Existence and multiplicity results for some superlinear elliptic problems on $\mathbf{R}^{N}$, Comm. Partial Differential Equations 20 (1995), 1725-1741. MR 1349229 (96f:35050)

3. T. Bartsch and Z.-Q. Wang, Multiple positive solutions for a nonlinear Schrödinger equation, Z. Angew. Math. Phys. 51 (2000) 366-384. MR.1762697 (2001f:35363)

4. T. Bartsch, A. Pankov and Z.-Q. Wang, Nonlinear Schrödinger equations with steep potential well, Commun. Contemp. Math. 3 (2001), no. 4, 549-569. MR1869104(2002k:35079)

5. A. Bahri and H. Berestycki, A perturbation method in critical point theory, Trans. Amer. Math. Soc. 267 (1981), 1-32. MR621969 (82j:35059)

6. A. Bahri and P. L. Lions, Morse index of some min-max critical points. I. Application to multiplicity results, Comm. Pure Appl. Math. 41 (1988), 1027-1037. MR.968487 (90b:58035)

7. V. Benci and G. Cerami, The effect of the domain topology on the number of positive solutions of nonlinear elliptic problems, Arch. Rational Mech. Anal. 114 (1991), 79-93. MR1088278 (91j:35102)

8. P. Bolle, On the Bolza problem, J. Diff. Eq. 152 (1999), 274-288. MR.1674537 (99m:58043)

9. H. Berestycki, Le nombre de solutions de certains problèmes semi-linéaires elliptiques, J. Funct. Anal. 40 (1981), 1-29. MR607588 (82k:35033)

10. D. Cao, E. S. Noussair, Multi-bump standing waves with a critical frequency for nonlinear Schrödinger equations, J. Differential Equations 203 (2004), no. 2, 292-312. MR2073688 (2005g:35265)

11. K. Cieliebak, E. Séré, Pseudoholomorphic curves and the shadowing lemma, Duke Math. J. 99 (1999), no. 1, 41-73. MR:1700739 (2000m:37130)

12. E. N. Dancer, Real analyticity and non-degeneracy, Math. Ann. 325 (2003), 369-392. MR.1962054 (2004h:35067)

13. E. N. Dancer, Some near critical problems, Adv. Differential Equations 8 (2003), no. 5, 571594. MR.1972491(2004e:35062) 
14. M. del Pino and P. Felmer, Local mountain passes for semilinear elliptic problems in unbounded domains, Calc. Var. Partial Differential Equations 4 (1996), no. 2, 121-137. MR.1379196 (97c:35057)

15. M. del Pino, P. Felmer and K. Tanaka, An elementary construction of complex patterns in nonlinear Schrödinger equations, Nonlinearity 15 (2002), 1653-1671 . MR 1925432 (2003g:35211)

16. Y. Ding and K. Tanaka, Multiplicity of positive solutions of a nonlinear Schrödinger equation, Manuscripta Math. 112 (2003), 109-135. MR2005933 (2004i:35099)

17. S. Fučík, M. Kučera, J. Nečas, J. Souček, V. Souček, Morse-Sard theorem in infinite dimensional Banach spaces and investigation of the set of all critical levels, Časopis Pěst. Mat. 99 (1974), 217-243. MR0370649(51:6875)

18. P. L. Lions, The concentration-compactness principle in the calculus of variations. The limit case. I. Rev. Mat. Iberoamericana 1 (1985), 145-201. II. Rev. Mat. Iberoamericana 1 (1985), 45-121. MR834360 (87c:49007)

19. Z. Nehari, Characteristic values associated with a class of non-linear second-order differential equations, Acta Math. 105 (1961), 141-175. MR0123775 (23:A1097)

20. P. H. Rabinowitz, Multiple critical points of perturbed symmetric functionals, Trans. Amer. Math. Soc. 272 (1982), 735-769. MR662065 (83k:35037)

21. P. H. Rabinowitz, Minimax methods in critical point theory with applications to differential equations. CBMS Regional Conference Series in Mathematics, 65, 1986. MR845785 (87j:58024)

22. B. Simon, Schrödinger groups, Bull. Amer. Math. Soc. (N.S.) 7 (1982), no. 3, 447-526. MR670130(86b:81001a)

23. M. Struwe, Infinitely many critical points for functionals which are not even and applications to superlinear boundary value problems, Manuscripta Math. 32 (1980), no. 3-4, 335-364. MR.595426 (82e:58030)

24. M. Struwe, A global compactness result for elliptic boundary value problems involving limiting nonlinearities, Math. Z. 187 (1984), 511-517. MR760051 (86k:35046)

25. K. Tanaka, Morse indices at critical points related to the symmetric mountain pass theorem and applications, Comm. Partial Diff. Eq. 14 (1989), 99-128. MR.973271 (90i:58019)

26. S. Terracini, G. Verzini, Solutions of prescribed number of zeros to a class of superlinear ODE's systems, NoDEA 8 (2001) 323-341. MR1841262 (2002f:34038)

Department of Mathematics, School of Science and Engineering, Waseda University, 3-4-1 Оhкubo, ShinuUku-Ku, TOKYo 169-8555, JAPAN

E-mail address: yohei-sato@aoni.waseda.jp

Department of Mathematics, School of Science and Engineering, Waseda University, 3-4-1 Ohкubo, Shinuuku-ku, Tokyo 169-8555, JAPAN

E-mail address: kazunaga@waseda.jp 